\title{
Structure and Dynamics of Molecular-Dynamics Simulated Undercooled Ni-Zr-Al Melts
}

\author{
Dissertation \\ zur Erlangung des Doktorgrades \\ der Mathematisch-Naturwissenschaftlichen Fakultäten \\ der Georg-August-Universität zu Göttingen
}

vorgelegt von

Mohammed Guerdane

aus Oran

Göttingen, 2000 
Referent: Prof. Dr. H. Teichler

Korreferent: Prof. Dr. K. Samwer

Tag der Mündlichen Prüfung: 01.11.2000 


\section{Contents}

\begin{tabular}{lll|}
\hline & Introduction & 3 \\
\hline
\end{tabular}

1.1 Bulk amorphous alloys $\ldots \ldots \ldots \ldots \ldots$

1.2 The diffusion mechanisms in amorphous alloys . . . . . . . . . . 4

1.3 To this work $\ldots \ldots \ldots \ldots \ldots \ldots \ldots \ldots$

\begin{tabular}{|lll}
2 & Molecular dynamics simulation methods & 9
\end{tabular}

2.1 Introduction . . . . . . . . . . . . . . . . . . . 9

2.2 MD simulation algorithms $\ldots \ldots \ldots \ldots \ldots$

2.3 Ensembles . . . . . . . . . . . . . . . . . . . . . . . 10

2.4 Periodic boundary conditions $\ldots \ldots \ldots \ldots \ldots$

2.5 Cut-off radius and Verlet-list . . . . . . . . . . . . . . . . 12

2.6 Samples preparation $\ldots \ldots \ldots \ldots \ldots \ldots \ldots$

$\begin{array}{llr}3 & \text { Interatomic Potentials } & 14\end{array}$

3.1 Pair-potential interactions $\ldots \ldots \ldots \ldots \ldots . \ldots . \ldots 14$

3.2 Many-atom interactions $\ldots \ldots \ldots \ldots \ldots \ldots$

3.3 Hausleitner-Hafner $(\mathrm{HH})$ interaction potentials $\ldots \ldots \ldots$. . . . . 16

3.4 Model used in this work $\ldots \ldots \ldots \ldots$. . . . . . . . . . 17

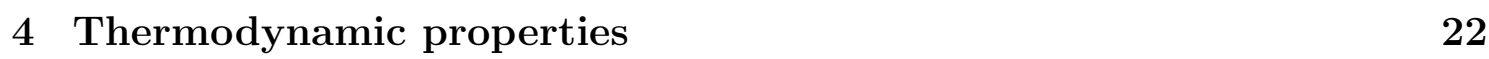

$\begin{array}{lll}5 & \text { Structural Properties } & 26\end{array}$

5.1 Pair distribution function $\ldots \ldots \ldots \ldots \ldots$

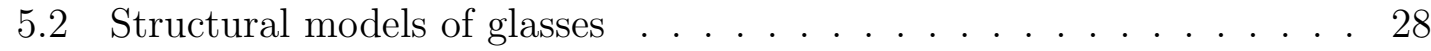

5.3 Comparison with experimental results . . . . . . . . . . . . . . 29

5.4 Bond-angle distribution $\ldots \ldots \ldots \ldots \ldots \ldots \ldots$

5.5 Radial distribution functions . . . . . . . . . . . . . . . . . . . 32

5.5 .1 Partial PDFs . . . . . . . . . . . . . . . . . . . 32

5.5 .2 Structural comparison with binary $\mathrm{Ni}_{40} \mathrm{Zr}_{60} \quad \ldots \ldots \ldots \ldots$

5.5 .3 On the thermal stability of amorphous $\mathrm{Ni}_{25} \mathrm{Zr}_{60} \mathrm{Al}_{15} \ldots \ldots 38$

5.6 Static Structure Factors and Short Range Order . . . . . . . . . . 38

5.7 Temperature dependence of the prepeaks $\ldots \ldots \ldots \ldots$. . . . . 42 
6 Dynamic properties $\quad 45$

6.1 Diffusion . . . . . . . . . . . . . . . . . . . . . 45

6.1 .1 Self-diffusion in the liquid state . . . . . . . . . . . . . . . . . 48

6.1 .2 Self-diffusion in the glassy state . . . . . . . . . . . . . . . . . 49

6.1 .3 Comparison with experimental results . . . . . . . . . . . . . 51

6.2 Van Hove Correlation function . . . . . . . . . . . . . . . . . . . . . . 51

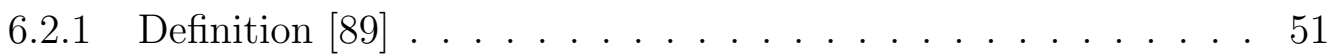

6.2 .2 Van Hove self correlation function . . . . . . . . . . . . . . . . 52

6.2 .3 Van Hove distinct correlation function . . . . . . . . . . . . . 56

6.3 Hopping and flow motion . . . . . . . . . . . . . . . . . . . . . . . . 58

6.4 Intermediate scattering function . . . . . . . . . . . . . . . . 60

6.5 Shear stress fluctuations and shear viscosity . . . . . . . . . . . . . . 65

6.6 Stokes-Einstein relation. . . . . . . . . . . . . . . . . . . 69

6.7 Diffusion and entropy . . . . . . . . . . . . . . . . . . . . . . . . 71

6.8 Comparison of dynamic properties in ternary ... . . . . . . . . . . 73

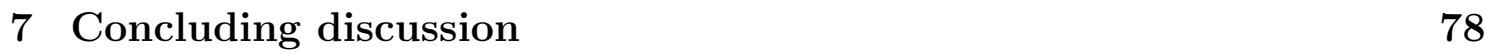

7.1 Glass transition . . . . . . . . . . . . . . . . . . . . . . 78

7.2 Prepeaks and medium-range Order . . . . . . . . . . . . . . . . . . . 79

7.3 Change in diffusion mechanism and decoupling behaviour . . . . . . . 80

7.4 Heterogeneity of the diffusion . . . . . . . . . . . . . . . . . . 81

7.5 Glass forming ability . . . . . . . . . . . . . . . . . . . . . 83

$\begin{array}{lll}8 & \text { Summary } & 86\end{array}$

\begin{tabular}{|lr}
\hline A k-dependent shear viscosity & 89
\end{tabular}

A.1 Definition . . . . . . . . . . . . . . . . . . . . . . . . . 89

A.2 Results . . . . . . . . . . . . . . . . . . . . . . . . 90

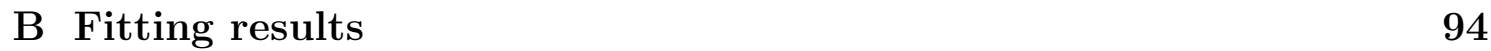




\section{Chapter 1}

\section{Introduction}

\subsection{Bulk amorphous alloys}

Development of new materials is an important activity in the continued progress of science and technology. Metallic amorphous alloys [1, 2, 3] are one such category of materials which have attracted the attention of scientists and technologists for the past three decades, during which these materials passed from a basic research interest to the status of practical materials for industriel applications. This is because of their excellent combination of characteristics resulting from their disordered structure. For instance, the amorphous alloys containing the transition metals $\mathrm{Fe}, \mathrm{Co}, \mathrm{Ni}$ as a major component exhibit extremely high tensile strengths which are about two times higher than those of crystalline alloys; some of them are exceptionally corrosion resistant; and they behave as very soft magnetic materials [3].

For the formation of an amorphous phase by rapid solidification (quenching) of the melt, it is essential to supress the nucleation and growth of crystalline phases in the supercooled liquid region, i.e. between the melting point $T_{m}$ and the glass temperature $T_{g}$. For the first generation of metallic glasses, up to the end of the eighties, critical cooling rates of the order of $10^{4}-10^{6} \mathrm{~K} / \mathrm{s}$ were required to form a glass by rapid solidification. In general, these high cooling rates have been reached by quenching the melts on a cold metallic substrate, which limits the sample geometry to thin ribbons of typically 50 to $100 \mu m$ in thickness. These ribbons show a low thermal stability with respect to crystallization when heated into the glass transition region. In many alloy systems, the glass transition cannot even be observed since the amorphous alloy transforms to a crystalline phase before $T_{g}$ is reached. As an exception, it was known that Pd- and Pt-based amorphous alloys have critical oooling rates of $10^{2}-10^{3} \mathrm{~K} / \mathrm{s}$ and are amorphized in the thickness range of about $4 \mathrm{~mm}$ by rapid quenching in water.

The realization of metallic amorphous alloys by very low cooling rates, comparable to those of oxide glasses, has been a long-cherished desire of materials scientists. This aim was realized at the begining of the nineties by the discovery of new 
families of multicomponent glass forming alloys such as Zr-Ni-Al [4], La-Ni-Al [5], Zr-Ni-Al-Cu [6], and Zr-Ti-Cu-Ni-Be [7]. These alloys show a high thermal stability of their supercooled liquid when heated above $T_{g}$. Moreover, cooling rates as low as $1-100 \mathrm{~K} / \mathrm{s}$ are sufficient to reach high levels of undercooling. Consequently, it becomes possible to produce bulk samples of about $10 \mathrm{~mm}$ (cm class) by conventional casting processes. This opens a new era for amorphous alloys because now one can overcome earlier limitations of shape and size which have been the most serious disadvantages for a broader use of these alloys.

These new bulk amorphous alloys are excellent advanced engineering materials due to their excellent wear properties, low coefficient of friction and corrosion resistance. Viscosities in the range of $10^{7}-10^{8}$ poise can be reached in the undercooled region below the crystallization temperature $T_{x}$. Therefore they can be easily deformed by pressing, drawing and blowing, processes known for the working of oxide glasses. Furthermore, the high thermal stability of these alloys makes for first time possible the detailed exploration of the kinetics and the thermodynamics in the supercooled region, up to about $100 K$ for some bulk amorphous alloys [8].

Various models have been proposed to explain the unusually large glass forming ability of the bulk metallic glass formers [9, 10]. These models can be grouped into three categories of thermodynamics, kinetics and structure. The three models are discussed in chapter 7. On the other hand, Inoue [3] advanced three empirical rules to characterize a bulk metallic glass, i.e., 1) multicomponent alloy system consisting of at least three components, 2) significant different atomic size ratios, above about $12 \%$, among the main constituent elements, and 3) negative heats of mixing among their elements. According to Inoue, fulfillement of these three conditions leads to the formation of a highly dense packed structure.

\subsection{The diffusion mechanisms in amorphous al- loys}

The nature of atomic transport in metallic glasses has been the subject of extensive theoretical and experimental studies over the past decade. Particularly, the basic diffusion mechanisms still remains a controversial discussed issue [21, 22]. This interest comes from the fact that diffusion controlls many processes in amorphous alloys such as structural relaxation, phase separation and crystallization which are of importance for predicting the thermal stability of these materials.

The material transport in ordinary liquids well above the melting point seems to be quite well understood. The diffusion takes place via a viscous flow as described by the Stokes-Einstein relation. Kinetic models predict a temperature dependence of the type $D \propto T^{n}$, where $n$ is found to be close to 2 according to MD simulations and experiments [23]. 
Upon cooling the liquid, its viscosity increases continously and the atoms become more and more trapped in their nearest-neighbor "cages" for times longer than the vibration time. The mode coupling theory (MCT) predicts that this cage effect leads to a freezing of the viscous flow at a critical temperature $T_{c}$, and that the diffusivity above $T_{c}$ obeys a power law dependence $D \propto\left|T-T_{c}\right|^{\gamma}[24$. The predictions of the MCT have been confirmed by several MD simulations [25, 26, 27]. It was shown, moreover, that around $T_{c}$ a change in the diffusion mechanism takes place, namely a transition from a viscous flow to thermally activated hopping [28, 29, 30].

In the glassy state, the basic diffusion mechanism still remains under discussion. A very important aspect is whether diffusion is essentially a single-jump process similar to defects (vacancies or interstitials) diffusion in crystalline solids or a collective process where many atoms are involved. The temperature dependence of the diffusion coefficient gives access to the activation energy and to the preexponential factor. These two parameters give, however, no information about the diffusion mechanism in the amorphous alloys studied. The key experiment, which gives more insight into this question, is to measure the isothermal pressure dependence of the diffusivity [21]. If the diffusion takes place via, e.g., a single-jump-type vacancy mechanism, one expects an activation volume, defined as $V_{a c t}=-k_{B} T(\partial \ln D / \partial p)_{T}$ , of the order of one atomic volume. The few measurements of the pressure dependence of diffusion performed until today lead to contradictory conclusions, since the activation volumes deduced range from $-0.06 \Omega$ to $1 \Omega$ ( $\Omega$ : atomic volume).

Höfler et al. [31] measured the effect of pressure on tracer diffusivity of Co in amorphous $\mathrm{Ni}_{\mathrm{x}} \mathrm{Zr}_{1-\mathrm{x}}$ and found an activation volume close to $1 \Omega$. Duine et al. [79] determined an activation volume for diffusion of $\mathrm{Au}$ in amorphous $\mathrm{Pd}_{40} \mathrm{Ni}_{40} \mathrm{P}_{20}$ also close to one atomic volume. From the similarity of these activation volumes with typical values in crystalline materials with diffusion via a vacancy mechanism, these autors proposed a defect mechanism for the amorphous structures, too.

In contrast, Faupel et al. 32] measured the pressure dependence of Co diffusivity in $\mathrm{Co}_{76.7} \mathrm{Fe}_{2} \mathrm{Nb}_{14.3} \mathrm{~B}_{7}$ and found $V_{a c t} \approx-0.06 \Omega$. Rummell et al. [80] also found an activation volume $V_{a c t} \approx-0.07 \Omega$ for Co diffusity in amorphous $\mathrm{Fe}_{40} \mathrm{Ni}_{40} \mathrm{~B}_{20}$ alloy. These vanishing activation volumes have been attributed to a highly collective diffusion mechanism involving the simultaneous rearrangement of several atoms. This observation has been related by Heesemann et al. [81] with the very small isotop effect of cobalt diffusion in the amorphous state of several conventional metallic glasses (e.g. Co-rich Co-Zr alloys) as well as in the deeply supercooled melt of the bulk glass forming alloy $\mathrm{Zr}_{41.2} \mathrm{Ti}_{13.8} \mathrm{Ni}_{10} \mathrm{Cu}_{12.5} \mathrm{Be}_{22.5}$.

That both diffusion mechanisms (the single-jump as well the collective process) are possible in amorphous alloys, is in agreement with findings of Teichler [30] who showed in MD simulations of arrested $\mathrm{Ni}_{80} \mathrm{Zr}_{20}$ and $\mathrm{Ni}_{50} \mathrm{Zr}_{50}$ that in the first sample about $80 \%$ of $\mathrm{Ni}$ diffusion take place in form of cooperative hopping processes of several atoms (ring mechanism), while in the second sample $85 \%$ of the Ni transitions 
are single atom jumps with flow motion in their environment.

\subsection{To this work}

Basic understanding of the material properties requires often knowledge on a microscopic level of the underlying energetics and interaction mechanism. In most situations, this knowledge is not accessible by experimental tools and the problem is too complex to be treated by analytical theories. In these situations, which include the majority of material systems and phenomena, the use of computer-based methods is unavoidable. Growth in computer power is allowing systems of increasing complexity to be studied, and refinements in interatomic potentials are leading to greater precision in the calculations.

In the present work, we use one of the popular computer-based methods, namely the molecular dynamics (MD) simulation, to model an amorphous alloy with a large glass forming ability (GFA). At the origin was the intention to understand how is this large GFA reflected in thermodynamic, structural and (or) dynamic properties of such an alloy. The availibilty of the microscopic information (atomic positions and velocities) makes possible the direct calculation of these properties.

The choice of the ternary bulk amorphous alloy $\mathrm{Ni}_{25} \mathrm{Zr}_{60} \mathrm{Al}_{15}$ has been dictated by methodical constraints. The realistic modellization of a physical system by MD simulation methods supposes the availability of reliable interatomic potentials for this system, or eventually the possibility to develop these potentials. This represents a complex problem in the case of bulk amorphous alloys since they are all multicomponent systems (more than two components). In the pair-potential picture,e.g., a system with $\mathrm{k}$ components requires that the $k(k+1) / 2$ pair-potentials are known. Regarding this, the choice of a ternary system is optimal. Moreover, the binary systems Ni-Zr have been widely studied in our group [11, 12, 13, 14, 15] by means of the Hausleitner-Hafner (HH) interatomic couplings [16]. The particular composition $\mathrm{Ni}_{25} \mathrm{Zr}_{60} \mathrm{Al}_{15}$ was chosen, as here experimental data concerning the thermodynamics and the structure are available. A comparison of these experimental results with those predicted by the model allows to check the reliabilty of the model. The particular interest of experimentators to this composition is due to its optimal GFA in the class of Ni-Zr-Al ternary alloys, as stated below.

The HH-potentials turned out to give a quite realistic description of the transition-metal Ni-Zr binary alloys. In our modelling of the ternary alloy $\mathrm{Ni}_{25} \mathrm{Zr}_{60} \mathrm{Al}_{15}$, we make further use of these potentials to describe the interatomic interactions in the subsystem Ni-Zr. The Al-Al interaction is described by the pair potential derived for pure $\mathrm{Al}$ within the framework of the pseudopotential theory [17. The remaining $\mathrm{Ni}-\mathrm{Al}$ and $\mathrm{Zr}-\mathrm{Al}$ interatomic couplings are determined by fitting a Stillinger-Weber analytical form [18] to experimental data of crystalline $\mathrm{Ni}_{3} \mathrm{Al}$ and $\mathrm{Zr}_{3} \mathrm{Al}$, respectively. 
Amorphous Ni-Zr-Al ternary alloys with a large glass forming ability were developed first by Inoue et al. 4]. The alloys exhibit a wide supercooled liquid region and a high mechanical strenght at the Zr-rich composition side. In particular, the alloy at the composition $\mathrm{Ni}_{25} \mathrm{Zr}_{60} \mathrm{Al}_{15}$ has a large temperature span $\Delta T_{x}=T_{x}-T g \approx 80 \mathrm{~K}$, which is the largest for these class of amorphous alloys. The highest value of the reduced glass transition temperature is also found in the vicinity of this composition with $T_{g} / T_{m} \approx 0.64$. The minimum cooling rate is in the range of $10^{2} \mathrm{~K} / \mathrm{s}$, which is comparable to that achieved by quenching in liquids.

In attempting to understand the high GFA of amorphous $\mathrm{Ni}_{25} \mathrm{Zr}_{60} \mathrm{Al}_{15}$ alloy, we carried out in this work a systematic comparison with the conventional binary glass former $\mathrm{Ni}_{35} \mathrm{Zr}_{65}$. For the $\mathrm{Zr}$-rich Ni-Zr binary amorphous alloys it has been reported that the glass transition is scarcely observed and that $\Delta T_{x}$ is below $10 \mathrm{~K}$. The comparison includes thermodynamical properties, the local atomic structure and the dynamical behavior.

Inoue et al. [4] suggested that the high thermal stability of Zr-rich Ni-Zr-Al ternary alloys compared to the Zr-rich binary ones originates partly from an increase in the packing fraction by the dissolution of $\mathrm{Al}$. Due to the intermediate atomic size of $\mathrm{Al}$ (the atomic radii of $\mathrm{Ni}, \mathrm{Al}$ and $\mathrm{Zr}$ are $1.24,1,43$ and $1.60 \AA$ ), it can fill up the vacant sites in the disordered $\mathrm{Zr}-\mathrm{Ni}$ structure. The dense atomic configuration is then thought to result in an increase of the viscosity (or equivalently a decrease of the diffusivity) of the system, leading to an enhanced thermal stability of the supercooled liquid. The maximal GFA of $\mathrm{Ni}_{25} \mathrm{Zr}_{60} \mathrm{Al}_{15}$ is explained by an optimal packing density at this composition. The dynamical study in Chapter 6 shows that in this point the predictions of our model disagree with the interpretations of Inoue et al.: We observe an enhanced diffusivity by substituting $\mathrm{Ni}$ by $\mathrm{Al}$ atoms for a given temperature. The analysis of the atomic-level stress tensor [103] points out that this effect is due to the n-defect (vacancy-like) character of the $\mathrm{Al}$ sites, which results in an increasing free volume by the dissolution of $\mathrm{Al}$. This effect may be explained by the higher binding strength of $\mathrm{Zr}-\mathrm{Ni}$ relatively to $\mathrm{Zr}-\mathrm{Al}$, as we will show in chapter 3 .

In Chapter 4 the systems are analysed in terms of thermodynamic quantities which are used to determine the caloric glass temperature $T_{g}$. A higher $T_{g}$ is found for the binary alloy compared to the ternary one. This is in agreement with the enhanced diffusivity obtained by substituting $\mathrm{Ni}$ through $\mathrm{Al}$ atoms.

In chapter 5 the atomic structure of the amorphous system is investigated in terms of the radial distribution function, the static structure factors and the bondangle distribution. A particular feature which emerges from this analysis is the existence of a pronounced chemical and topological short range order (SRO) even at very high temperatures. In a comparative study of a selection of Zr-based bulk metallic glasses (so-called Johnson glasses), Busch et al. 19] related the large GFA of these glasses with the presence of a chemical SRO. This suggestion is due to the ob- 
servation that glass formers with the lowest critical cooling rates have smaller Gibbs free energy differences $\Delta G_{l-x}$ between the undercooled liquid and the corresponding cristalline mixture. This mainly comes from a smaller entropy of fusion which determines the slope of $\Delta G_{l-x}$ at the melting point. Atom probe field microscopy and small angle neutron scattering experiments have confirmed the existence of such chemical SRO [20].

Chapter 6 deals with the dynamical properties of the studied systems. We investigate for this purpose the self-diffusion, the van Hove correlation functions, the intermediate scattering function and the shear viscosity for ternary $\mathrm{Ni}_{25} \mathrm{Zr}_{60} \mathrm{Al}_{15}$. This investigations are performed over a wide temperature range from the liquid state at higher temperatures down to the arrested (glassy) state. We test particularly some predictions of the MCT. We find that the transition in the diffusion mechanism from viscous flow to hopping processes starts already well above the critical temperature $T_{c}$. This transition is found to be associated with the decoupling of the diffusity from the viscosity, as expressed by the failure of the Stokes-Einstein relation. At the end of the Chapter a comparison with the binary $\mathrm{Ni}_{35} \mathrm{Zr}_{65}$ alloy is presented by analysing the atomic-level stress tensor. 


\section{Chapter 2}

\section{Molecular dynamics simulation methods}

\section{$2.1 \quad$ Introduction}

Basic understanding of the material properties often requires knowledge of the underlying energetics and interaction mechanism on a microscopic level. In most situations this knowledge is not accessible to experimental tools. In these cases, which include the majority of material systems and phenomena, the use of computer-based techniques [33, 34] seems to promise particular success. They make it possible to obtain rigorous numerical results about a complex system. Another merit of the computersimulation methods is that they can produce 'macroscopic' behavior under precisely controlled conditions which can be varied by the observator, and by so doing one can gain a great deal of physical insight into complex phenomena where several competing processes are in play. For instance, it is possible to consider the dependence of the glass properties on quench rates or on interatomic potentials. Nowadays, the computer simulations are successfully applied to a wide range of problems in solid state physics, chemistry, material sciences and quantum field theory, opening so a new branch of investigations besides the experimental and theoretical methods.

While, in the early days, the computer simulations were limited to small systems with several hundred particles, large scale simulations are today possible and new records in system sizes are reported frequently [35]. This is promoted by the remarkable progress in computer performance in the last two decades, especially with the generation of the powerfull parallel computers [36, 37].

In the present work, we make use of the molecular dynamics (MD) method, which is one of the computer-simulation techniques. A brief description of this method is given in the following sections. A more detailed representation can be found in references [38, 39, 40]. 


\subsection{MD simulation algorithms}

In MD simulations for a system of $N$ particles, phase space exploration is achieved by numerical integration of the $3 N$ coupled Newton's motion equations:

$$
m_{i} \frac{d^{2} \mathbf{r}_{i}}{d t^{2}}=-\nabla_{i} \Phi\left(\mathbf{r}_{1}, \mathbf{r}_{2}, \ldots, \mathbf{r}_{N}\right)
$$

in discret time steps $\Delta t$ (we used in this work $\Delta t=2.5 f s$ ) and a given set of thermodynamic variables (such as volume, pressure and temperature). $m_{i}$ denotes the mass of the ith particle, $\left\{\mathbf{r}_{i}\right\}$ the position vector of the particle and $\Phi\left(\left\{\mathbf{r}_{i}\right\}\right)$ the potential energy of the system, oftenly written as the sum of interatomic pair potentials.

The time evolution of the system is determined, through a deterministic calculation of the trajectory of each particle in the phase space. This is in contrast to the Monte Carlo method [38], where the system evolves according to stochastic dynamics by setting up a random walk to sample configurations via a given distribution function.

In order to solve the Eq. 2.1), we use a modified fifth-order Gear algorithm, which is described in reference [44]. Gear algorithms [43] refer to a class of predictorcorrector methods which require only one force evaluation per integration step $\Delta t$. This requirement is decisive when algorithms choice is made, since the force calculations take up the lion's share of the hole computing time. The fifth-order Gear algorithm uses explicitly positions and velocities of one step and forces at the three previous steps. Thus, derivatives with time up to the forth order are implicitly involved. This corresponds to an error of $(\Delta t)^{5}$ in the particles trajectories. Since indirectly higher order of derivative of the potential are involved in our algorithm, numerically rather stable solutions are obtained. The price we pay for such high accuracy is an extended memory storage space, which amounts 6 data per degree of freedom. For purpose of comparison: a velocity-Verlet-predictor-corrector algorithm requires storage of 4 data per degree of freedom, but has an accuracy of $(\Delta t)^{3}[34]$ only.

\subsection{Ensembles}

In the traditional microcanonical MD simulations, one considers the movement of a constant number of particles in a box whose size and shape are fixed. The system is assumed to be free from any external force so that the total energy is conserved. This ensemble is easy to handle, however it makes direct comparison with experimental results difficult since laboratory experiments are usually carried out at constant pressure.

In this work, all the simulations are performed under constant pressure $P$ and temperature $T$. This corresponds to the canonical ensemble $(N, P, T)$ in statisti- 
cal mechanics. In this ensemble, the total energy is allowed to fluctuate by the interaction with a piston and through the thermal contact with a heat bath. The temperature $T$ is related classically to the kinetic energy. We use this relation to realize the isothermal condition by scaling the velocities $\mathbf{v}_{i}$ of the particles

$$
\mathbf{v}_{i} \longrightarrow \mathbf{v}_{i} \sqrt{3 N k_{B} T / \sum_{i=1}^{N} m_{i} \mathbf{v}_{i}^{2}},
$$

in every time step $\Delta t$ of the simulation. This simple velocity-scaling method, due to Woodcock [44], represents an approximation to the gaussian constraint method [45], which realizes the correct canonical distribution in the coordinate space. The error of this approximation is of $\Delta t$, if the scaling is carried out at every time step.

The pressure $P$ is expressed as the average of the kinetic energy and the virial

$$
P=\frac{1}{3 V} \sum_{i=1}^{N}\left(m_{i} \mathbf{v}_{i}^{2}-\sum_{j>i}^{N} \frac{\partial \Phi\left(r_{i j}\right)}{\partial r_{i j}} r_{i j}\right)+P_{e x}
$$

where $\mathbf{v}_{i}$ is the velocity of the particle $i, \mathbf{r}_{\mathbf{i j}}$ the vector joining particle $i$ to particle $j$, and $\mathbf{F}_{\mathbf{i j}}=-\partial \Phi\left(r_{i j}\right) / \partial \mathbf{r}_{\mathbf{i} \mathbf{j}}$ the force exerted by particle $j$ on particle $i$. $P_{e x}$ is the external pressure, set to zero in our simulation. From Eq. (2.3) we see that the pressure can be adjusted by expanding or contracting the simulation box.

A more refined version of the constant pressure method was presented by Anderson [46]. In this approach the volume is a fluctuating degree of freedom with corresponding kinetic and potential energy term in the total Hamiltonian of an extended system. The equations of motion of the volume realize a feedback mechanism which keeps the pressure around a constant value in a natural way. In a similar way, Nosé [45] treated the constant temperature condition by adding a degree of freedom which describes the coupling to the heat bath. Parrinello and Rahman [47] extended this technique to allow a change of the shape of the simulation box. This method turned out to be a powerful tool to study the polymorphic phase changes in crystals, e.g. martensitic transformations [122].

\subsection{Periodic boundary conditions}

Two limitations are imposed to MD simulations in the realization of the thermodynamic limit. On one side, there are finite-size effects in the simulations in the sence that the numerical system with a typical particles number $N$ in the range of $10^{3}$ is necessarly limited in size. A system of this size would be considerably affected by surface effects. On the other side, there are finite-observation-time effects in the sence that the simulation time is much shorter than the time used in experimental measurements. In this work, we use the conventional periodic boundary conditions (pbc) to minimize the finite-size effects. This approach consists in a periodic repetition of the simulation box in the three directions to fill the whole space. This 
operation removes all free surfaces. By construction, each particle possesses an infinity of periodic images. To avoid that a particle interacts with its own image or with two images of the same particle, the minimal-image convention is adopted [38, 39, 40]. Only interaction with the closest images is taken into account in this scheme. The range of the interaction is assumed to be smaller than $L / 2$, where $L$ is the length of the cubic box.

In order to minimize the finite-observation-time effects, the desired physical properties are evaluated by averaging the results over different configurations and over several MD runs using different starting configurations.

\subsection{Cut-off radius and Verlet-list}

In modelling of metals, short-ranged potentials are usually used. The advantage of such potentials in MD simulations is evident: In a relatively large system, the number of particles pairs to be considered in the laborious forces calculations could be considerably reduced. In this work, we describe the interatomic potentials mainly with Stillinger-Weber [18] pair-functions which decay smoothly to zero at a distance $r_{c}$, the cut-off radius. For a given atom, the interactions are thus limited to the neighbors at distances smaller than $r_{c}$. The calculation of the different $N(N-1) / 2$ distances in every integration step consumes a significant computing time. This can be considerably reduced by making use of the Verlet-list [40]. To every atom one attributes a neighbor list which contains all particles within a Verlet-radius $r_{v}$, choosen somewhat larger than $r_{c}$. In calculating the forces acting on a given atom, only the particles belonging to its list are considered. The neighbor list is updated once every about 5 to 20 integration steps, depending on the mobility of the particles, such that the estimated maximal displacement of the particles between two updating remains smaller than the difference $r_{v}-r_{c}$.

\subsection{Samples preparation}

The simulations presented in this work have been performed with $N=1100$ atoms for the ternary system $\mathrm{Ni}_{25} \mathrm{Zr}_{60} \mathrm{Al}_{15}$ and $N=648$ atoms for the binary $\mathrm{Ni}_{35} \mathrm{Zr}_{65}$. Starting with a liquid configuration at $3000 K$, well relaxed structures have been generated at lower temperatures by cooling and equilibrating the system. The equilibration time lies between about $0.8 \times 10^{6}$ integration steps $(2 \mathrm{~ns})$ at highest temperatures and $20 \times 10^{6}$ integration steps $(50 \mathrm{~ns})$ at lowest temperatures. In order to evaluate the various physical parameters, data analysis was carried out over about the last two third of the equilibration run. Taking into account the equilibration time, the effective cooling rate is of the order of $10^{10}-10^{9} \mathrm{~K} / \mathrm{s}$, which is much larger than the cooling rates usually achieved in experiments but still smaller than those 
often used in previous MD simulations. 


\section{Chapter 3}

\section{Interatomic Potentials}

The degree of correspondance between the MD simulation models and the physical system of interest depends to a large extent on the nature of the potential energy employed in the simulations. The use of reliable potentials is therefore of a central importance for atomistic simulations.

In the simple models, the interaction between the atoms are described with pairwise interactions, the so-called pair potentials. These potentials have known deficiencies (e.g., a rigid but unrealistic correlation between cohesion and vacancy formation energy) which can be removed partly by going beyond the pair-potential picture and introducing some many-atom interactions. We give in the following a brief decription of some of these potentials [48].

\subsection{Pair-potential interactions}

There are two different approaches to describe the interatomic interactions in terms of pair potentials. The first approach is to write the total energy of an atomic arrangement as a sum over simple pair potentials

$$
E_{T o t}=-\frac{1}{2} \sum_{i \neq j} \Phi_{i j}\left(\mathbf{R}_{i}-\mathbf{R}_{j}\right),
$$

where $\Phi$ defines the pair potential and $\mathbf{R}_{i}$ the atom coordinates. Rare gases and ionic systems, for example, can be adequatly described in terms of these pair potentials. They include the Lennard-Jones potentials

$$
\Phi(r)=4 \epsilon\left(\left(\frac{\sigma}{r}\right)^{12}-\left(\frac{\sigma}{r}\right)^{6}\right),
$$

commonly used for rare gases. A variety of other pair potentials have been suggested to describe materials other than rare gases. A widely used example is the Morse potential

$$
\Phi(r)=\epsilon\left(e^{-2 \alpha\left(r-r_{0}\right)}-2 e^{-\alpha\left(r-r_{0}\right)}\right),
$$


which has three parameters to be fixed to the experimental properties of the materials in question.

The deficiency of simple pair potentials lies in their inability to reproduce many important properties of metals, e.g. the vacancy formation energy and the relation between the elastic constants (Cauchy relations).

The second approach to pair-potential description of the total energy is based on the linear response theory and is mainly used in simple metals. In nearly-freeelectron-like simple metals (nontransition metals) the valence electrons form an homogeneous electron gas and the ions can be described by weak pseudopotentials. In the second order perturbation theory the total energy of the metal has the form [49]

$$
E_{T o t}=-\frac{1}{2} \sum_{i \neq j} \Phi_{i j}\left(\mathbf{R}_{i}-\mathbf{R}_{j}\right)+E_{V o l},
$$

where $E_{V o l}$ is an energy term independent of the structure of the metal but dependent on the total volume of the system. In this approach, the pair pseudopotential $\Phi$ has a very different meaning than the expression of Eq. 3.1 since only part of the total energy comes from the sum over the pair potentials (in Al, for example, the pair-potential sum gives only a few percent of the cohesion energy). Generally, the interatomic potentials based on the pseudopotential approach work fairly well for those properties of the metals where the density fluctuations are small. In the case of surfaces and open volume defects, such as vacancies, these potentials are, however, inappropriate since the density changes are so large that they cannot be described by the linear response theory.

\subsection{Many-atom interactions}

For metallic systems, an alternative to the pair-potential picture is provided by the effective medium theory (EMT) [51] or the related embedded atom method (EAM) [52]. This approach is based on the ideas of the density-functional theory [50] (and amenable to MD-simulation modelling). Here the total energy of the metal can be written as

$$
E_{T o t}=\sum_{i} F\left(n_{i}\right)+\frac{1}{2} \sum_{i \neq j} \Phi_{i j}\left(\mathbf{R}_{i}-\mathbf{R}_{j}\right),
$$

where $n_{i}$ is an effective electron density at the site of the atom $\mathrm{i}$ and is approximated by the superposition of atomic density tails from the other atoms

$$
n_{i}=\sum_{i} n_{a t, i}\left(\left|\mathbf{R}_{i}-\mathbf{R}_{j}\right|\right) .
$$

$F\left(n_{i}\right)$ is a nonlinear function (the "embedding energy") describing the interaction of the atom $i$ with its electronic surrounding, and $\Phi$ a pair potential. The embeddingenergy term $F\left(n_{i}\right)$ is supposed to describe the complex many-atom interactions. 
Moreover, it is an explicit function of atomic positions so that variations in local atom density can be easily accounted for. $F, n_{i}$ and $\Phi$ can be derived theoretically or determined empirically by choosing proper parametrization functions and fitting the parameters to some experimental data.

The first empirical method, in the form of Eq. (3.5), was the EAM model introduced by Daw and Baskes [52]. In their formulation, the pair potential $\Phi$ is a purely repulsive term accounting for ion-ion and orthogonalization repulsion at short range. Since this original work, several other methods, equivalent to the EAM, have been proposed. These methods all take the form of Eq. 3.5 with differences due to specific parametrizations of $F$ and $n_{i}$. Finnis and Sinclair [53] proposed that the $\mathrm{d}$-d hybridization in the second-moment approximation of the tight-binding model could also be described by Eq. (3.5). The energy function $F$ is found then to be negative and can be thought to be proportional to the negative square root of the coordination number.

The advantage of the total energy expression of Eq. (3.5) is that it is essentially as efficient in computer simulations as the pair-potential expressions of Eq. (3.1) or Eq. (3.4). Moreover, EAM potentials have been found to describe fairly well bulk and surface properties of transition metals. Similar good results have been obtained using the tight-binding model of Finnis and Sinclair [53].

Moriarty [54] has extended the pseudopotential theory to higher order of perturbation to derive three-body forces and higher many-body interactions to the elemental transition metals. This method has been applied by Rössler [55] to reproduce successfully the structural properties of a range of transition-metals melts. However, as this approach is based on a uniform electron gas as starting point, it is not expected to be adequate, even in higher orders, for studying inhomogeneous defect environments; e.g. the structure of surfaces where the electron density goes to zero.

\subsection{Hausleitner-Hafner (HH) interaction poten- tials}

Hausleitner and Hafner [56] presented a hybridized nearly-free-electron (NFE) tightbinding-bond (TBB) approach to interatomic forces in disordered (liquid and glassy) transition-metal alloys. The basic assumption of this model is to divide the total energy into contributions from the localized strongly bonded d-electrons and the much more mobile s-electrons

$$
E_{T o t}=E_{s}+E_{d} \text {. }
$$

The s-electrons contribution is treated in a NFE approximation. The pseudopotential perturbation theory here is used to write $E_{s}$ in terms of a volume-energy term 
and a sum over pair potentials, Eq. (3.4). The d-electrons contribution is written within the TBB approximation in terms of a repulsive pairwise interaction containing the electrostatic, exchange-correlation and the nonorthogonality contributions and a covalent bond energy resulting from the formation of $\mathrm{d}$ band

$$
E_{d}=E_{d, r e p}+E_{d, b o n d} .
$$

Hybridization between s and d states is taken into account by setting the numbers of s and d electrons equal to the values resulting from a self-consistent band-structure calculation for the pure crystalline transition metals.

The repulsive energy $E_{d, r e p}$ is assumed, after Wills and Harrison [58, to be given by a sum over pair potentials with a power-law dependance $E_{d, r e p} \sim \frac{1}{R^{8}}$. $E_{d, \text { bond }}$ can be written in a two-centre orthogonal tight-binding approximation as

$$
E_{d, b o n d} \sim \sum_{i, j} h\left(\left|\mathbf{R}_{i}-\mathbf{R}_{j}\right|\right) \theta_{i j}
$$

where $h$ is the transfert integral for $\mathrm{d}$ orbitals centered at sites $i$ and $j . \theta_{i j}$ is the bond order parameter which is defined as the difference between the number of electrons in the bonding and the antibonding states formed by the orbitals at sites $i$ and $j$. Eq. (3.9) represents only formally a pair interaction, the quantummechanical many-body character of the covalent bond enters via the bond order $\theta_{i j}$ which depends on the local atomic environment.

The hybridized NFE-TBB approach has been applied by Hausleitner et al. [57] and by Rössler [55, 59] in atomistic modelling of binary (Fe, Co, Ni) $(100-\mathrm{x}) \mathrm{Zr}_{\mathrm{x}}$ glasses via MD simulations. The approach is able to explain successfully the significant details of structural, of electronic and of magnetic properties of these amorphous structures.

\subsection{Model used in this work}

We describe in this subsection the interatomic potentials employed in this work to study the NiZrAl system.

First we adopt a global form of the total energy similar to that one derivated in the NFE-TBB approach; i.e., effective pairwise interactions augmented by a volumedependent free-electron term $E_{V o l}$. We use for $E_{V o l}$ the energy expression of a homogeneous electron gas perturbated to first order by the ionic lattice as known from the theory of cohesion of simple metals [60].

Concerning the effective pair potentials, a hybrid of different approaches has been adopted. For the $\mathrm{Ni}-\mathrm{Zr}$ subset we use the pair potentials as derived by Hausleitner and Hafner for the amorphous binary alloy $\mathrm{Ni}_{35} \mathrm{Zr}_{65}$ in the framework of the NFE-TBB scheme [56]. This concentration has been choosen because it is close to the relative concentration of $\mathrm{Ni}$ and $\mathrm{Zr}$ in the $\mathrm{Ni}_{25} \mathrm{Zr}_{60} \mathrm{Al}_{15}$ system modelled 
in this work. Hausleitner and Hafner showed, moreover, that in this concentration range the pair potentials exhibit only a weak concentration dependence, so that slight concentration deviation from that of $\mathrm{Ni}_{35} \mathrm{Zr}_{65}$ alloy could be modelled with the same pair potentials without significant effects on the obtained results.

As proposed by Teichler [11, the HH-potential is fitted by a Stillinger-Weber form

$$
\Phi_{i j}(r)=A\left(\left(\alpha r-a_{1}\right)^{-n}-1\right) \exp \left(\frac{1}{\alpha r-a_{2}}\right) \quad\left(r<a_{2} / \alpha\right) .
$$

The parameters are adapted to the minimum position, depth, width and zero of the pair potentials. The analytical form of Eq. 3.10 has the advantage to need less computation time than the original potentials and is therefore more appropriate for long-time runs. The SW form has, moreover, the advantage to go smoothly to zero at a reasonable cutoff distance and that the function and its derivatives have no discontinuities in $r$. The use of the original potentials would require to cut them abruptly at a given distance because of their relatively long-range nature.

The resulting SW fitting-parameters for the different atomic pairs of the $\mathrm{Ni}-\mathrm{Zr}$ subset are given in tab. (3.1), the corresponding potentials are shown in Fig. (3.1).

The pair interaction $\Phi_{A l A l}$ is described in our model by the pair potential derived for pur $\mathrm{Al}$ within the framework of the second order pseudopotential theory [61]. The use of such a first principle potential requires usually tabulation at each atomic volume. Alternatively, we use hier the following analytical form

$$
\Phi(r)=2 \frac{Z^{2}}{r} \sum_{n=1}^{3} A_{n} \cos \left(k_{n} r+\alpha_{n}\right) e^{-\kappa_{n} r},
$$

suggested by Pettifor et al. [17], which is well suited for use in MD simulations. The parameter values are given in tab.(3.2). As this potential is of a long-range nature (due to the asymptotic Friedel oscilllations), we impose a cutoff radius at a distance of $5.5 \AA$.

At this stage, the remaining cross interaction potentials $\Phi_{N i A l}$ and $\Phi_{Z r A l}$ need to be determined. We assume these potentials to have the empirical form of StillingerWeber, Eq. (3.10). The parameters (five for each potential function) are then fitted to the experimental values of the cohesive energy, the bulk modulus and the lattice constant of the cristalline structure $\mathrm{Ni}_{3} \mathrm{Al}$ (fcc) and $\mathrm{Zr}_{3} \mathrm{Al}(\mathrm{fcc})$. The contributions of nearest-neighbors and second nearest-neighbors are taken into account in the fitting procedure. Additionnaly, we imposed the equilibrium condition, which involves the first derivative of the cohesive energy with respect to volume. The physical quantities used for the fit for the two cristalline systems are listed in tab.(B.1) together with the values resulting from the fitting. The last square fit was performed by employing the software minimization package NAG [62] . The SW-parameters for the pair 
potentials $\Phi_{N i A l}$ and $\Phi_{Z r A l}$ are given in tab.(3.1). The corresponding curves are provided in Fig. (3.1)

An important feature of the pair potentials in Fig. (3.1) is their non-addivity character. Moreover, in each subset (Ni-Zr,Ni-Al and $\mathrm{Zr}-\mathrm{Al}$ ) the interaction between the pair of unlike atoms is found to be stronger than the mean interaction in likeatom pairs

$$
\Phi_{A B}(r)<\frac{1}{2}\left|\Phi_{A A}(r)+\Phi_{B B}(r)\right|,
$$

for $r$ around the potential-minimum distances. This means a compound formation tendency or a heterocoordination preference (preference for the formation of unlikeatom pairs) in the three binary subsets [61. This trend is experimentally well established for the three binary compounds as reflected by a pronounced negativity of their respective heats of mixing and a strong tendency to short-range order in their structure [63, 64, 65]. Moreover, the heterocoordination seems to be stronger for the binary systems $\mathrm{Ni}-\mathrm{Al}$ and $\mathrm{Ni}-\mathrm{Zr}$ than for Zr-Al. This feature implies that there may be a competing mechanism in the chemical short-range order in the ternary alloy $\mathrm{Ni}-\mathrm{Zr}-\mathrm{Al}$ as it will be discussed in section (5.5).

Table 3.1: Stillinger-Weber potential-parameters (Eq. (3.10))) used for the atom pairs indicated in the table.

\begin{tabular}{cccccc}
\hline \hline & $A[\mathrm{eV}]$ & $\alpha\left[\AA^{-1}\right]$ & $a_{1}$ & $a_{2}$ & $\mathrm{n}$ \\
\hline $\mathrm{Ni}-\mathrm{Ni}$ & 1.150 & 0.393 & 0 & 1.527 & 5 \\
$\mathrm{Ni}-\mathrm{Zr}$ & 3.350 & 0.365 & -0.128 & 1.672 & 5 \\
$\mathrm{Zr}-\mathrm{Zr}$ & 5.166 & 0.337 & 0 & 1.854 & 3 \\
$\mathrm{Ni}-\mathrm{Al}$ & 3.060 & 0.429 & 0 & 1.716 & 3 \\
$\mathrm{Zr}-\mathrm{Al}$ & 1.305 & 0.339 & -0.068 & 1.830 & 12 \\
\hline \hline
\end{tabular}


Table 3.2: Pair-potential parameters (Eq. (3.11) for pur Al after Pettifor et al. [17].

\begin{tabular}{ccccc}
\hline \hline $\mathrm{n}$ & $A_{n}[\mathrm{eV}]$ & $k_{n}[\AA]$ & $\kappa_{n}[\AA]$ & $\alpha_{n} / \pi$ \\
\hline 1 & 57.317 & 0.546 & 2.776 & -0.441 \\
2 & 9.176 & 2.254 & 2.443 & 0.832 \\
3 & 0.216 & 3.353 & 0.977 & 0.431 \\
\hline \hline
\end{tabular}




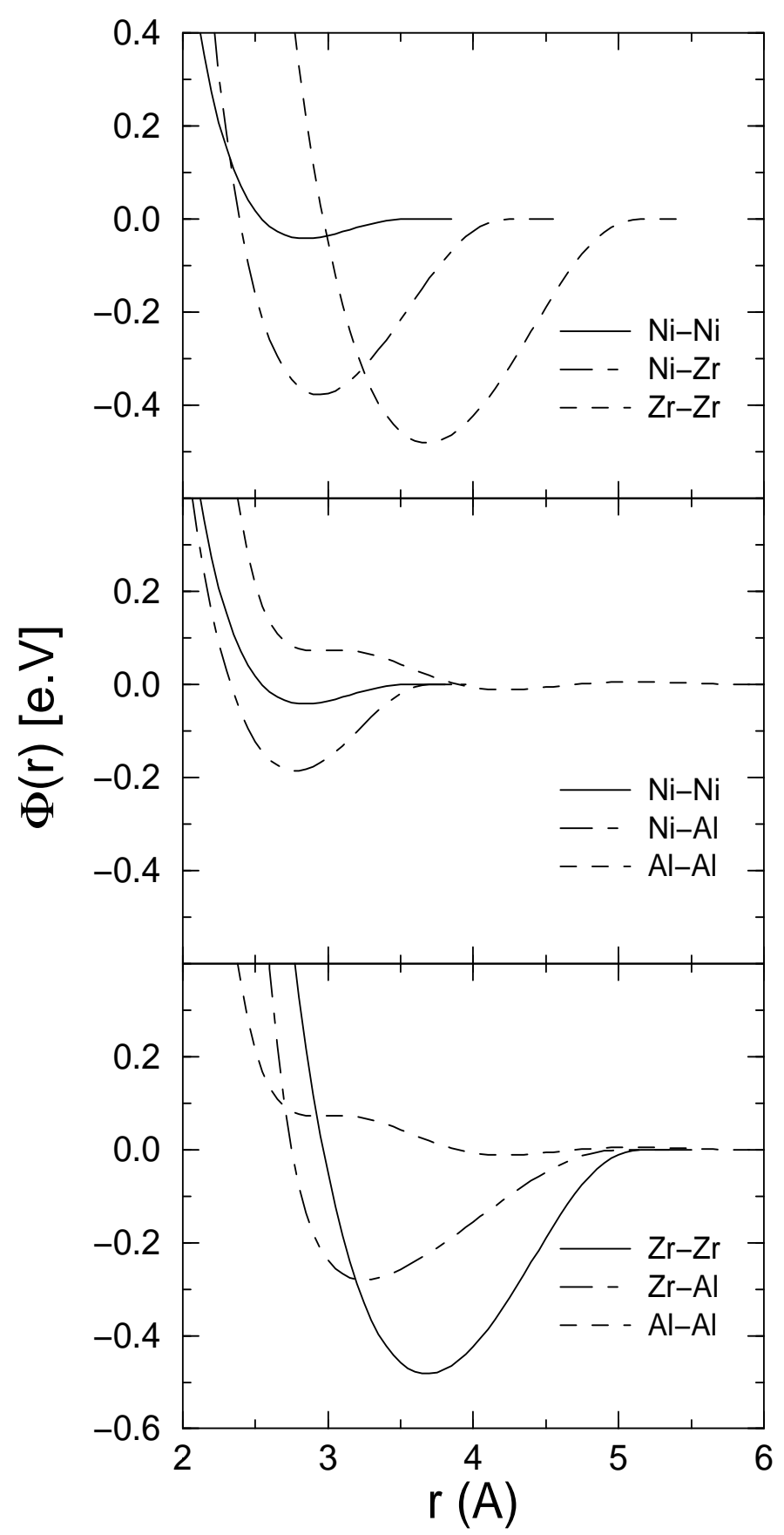

Figure 3.1: Pair potentials used in this work. For clarity, the pair potentials of the three binary subsystems $\mathrm{Ni}-\mathrm{Zr}, \mathrm{Ni}-\mathrm{Al}$ and $\mathrm{Zr}-\mathrm{Al}$ are presented separately. 


\section{Chapter 4}

\section{Thermodynamic properties}

In this section, we characterize the systems in terms of simulated thermodynamic quantities. We plot, therefore, in Fig. (4.1) and Fig. (4.2) the temperature dependence of the enthalpy per atom reduced by the energy of the harmonic oscillator, $H-3 k_{B} T$, and the volume of the cell $V$. Experimentally and from MD simulation, these quantities are found to undergo significant changes in the glass transition region. The changes manifest themselves in our simulations at the temperature $T \approx 1025 K$ for the ternary system and at $T \approx 1200 K$ for the binary one. These temperatures we identify as the caloric glass temperatures for the corresponding model systems.

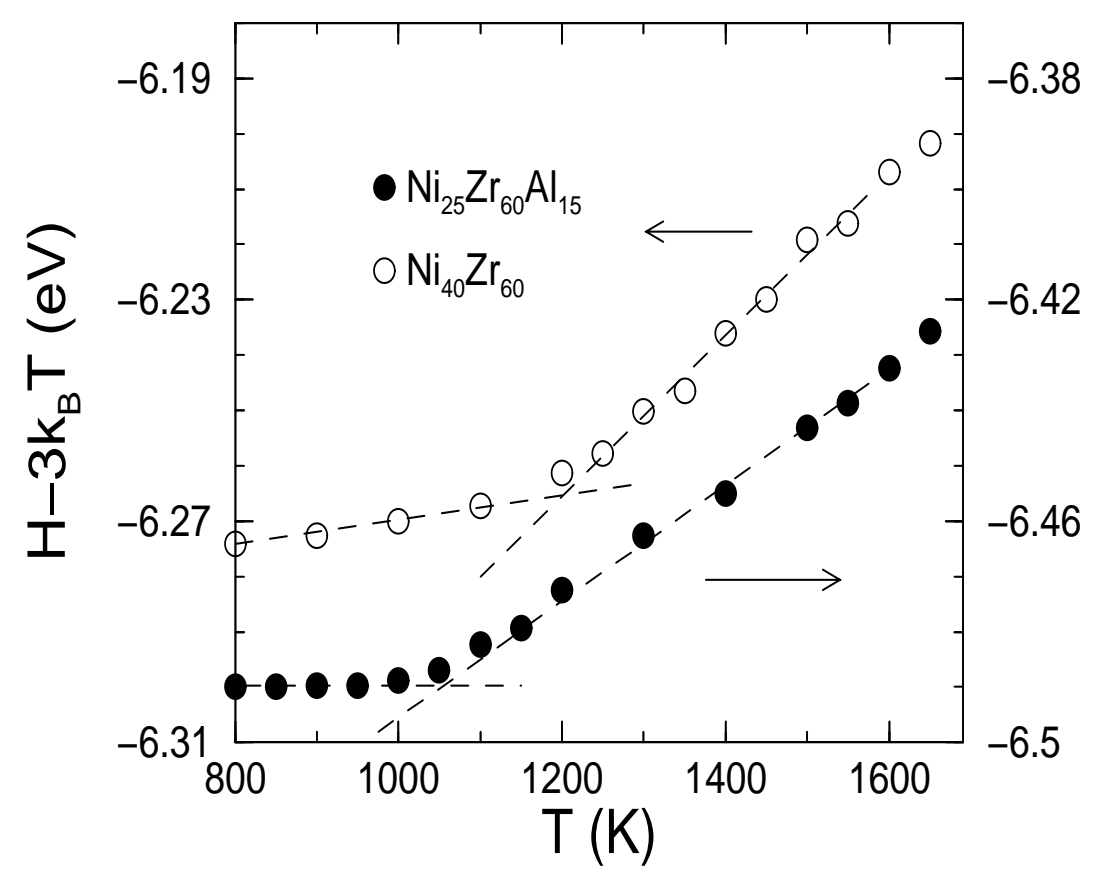

Figure 4.1: Enthalpy $H-3 k_{B} T$ per particle versus temperature $\mathrm{T}$ for the ternary $\mathrm{Ni}_{25} \mathrm{Zr}_{60} \mathrm{Al}_{15}$ and binary $\mathrm{Ni}_{40} \mathrm{Zr}_{60}$ alloys.

It is assumed that the glass transition (GT) occurs when the typical relaxation time of the system exceeds the time scale of probing the system. Moreover, it has 
been established experimentally and by MD simulations [123, 124] that the higher the cooling rate, the higher the glass temperature $T_{g}$. This confers a kinetic aspect to the glass transition.

Since in our case the cooling rates used for both systems are comparable, a higher $T_{g}$ for the binary system suggests, thus, that the time scale of the dynamic processes, for a given temperature in the undercooled state, is larger in the binary system than in the ternary one. This statement will be confirmed from the dynamic analysis in chapter 2. The shift of $T_{g}$ towards lower temperatures by alloying Al to Ni-Zr alloy can be attributed here to the higher binding strength of $\mathrm{Zr}-\mathrm{Ni}$ relatively to $\mathrm{Zr}-\mathrm{Al}$.

By differentiating the enthalpy $H$ with respect to the temperature, we obtain $C_{p}$, the specific heat at constant pressure, which provides more details on the variation of the energy of the system with temperature. $C_{p}$ can be alternatively evaluated from the time average fluctuations of the enthalpy at a given temperature([66])

$$
<(\delta H)^{2}>=<H^{2}>-<H>^{2}=k_{B} T^{2} C_{p} .
$$

The validity of this formula supposes that the system is in thermodynamic equilibrium. This second method is particularly useful when the enthalpy data are spoilt by noise or are not sufficiently reliable to allow the evaluation of the derivative.

The values of $C_{p}$ at temperatures above $T_{g}$ are calculated here by using the fluctuation formula Eq. (4.1). For temperatures below $T_{g}$, this formula turns out to give underestimated values of $C_{p}$, which is probably due to the fact that for $T<T_{g}$ the simulation time scale is too short to take care of all possible fluctuations of the system. Therefore, we evaluated $C_{p}$ in this temperature range from the direct differentiation of the enthalpy. The results are ploted on Fig. (4.3). Additionally we show the experimental results $C_{p, \exp }$ of Inoue et al. [4] from the differential scanning calorimetry (DSC) measurements for $\mathrm{Ni}_{25} \mathrm{Zr}_{60} \mathrm{Al}_{15}$ amorphous alloy with a heating rate of $0.67 \mathrm{~K} / \mathrm{s}$.

We see that $C_{p}$ increases slowly upon cooling from high temperatures down to the GT regions, where it drops abruptly. This pronounced drop is conventionally associated with the glass transition. $C_{p, \exp }$ exhibits a similar behavior. One observes a continuous increase under heating from low temperatures up to the GT region, followed by a decrease in the supercooled region bevor it drops rapidly due to the onset of cristallization. The direct comparison of $C_{p, \exp }$ and calculated $C_{p}$ is not possible owing to the large distance between the GT regions in the experiment and the MD simulation and the onset of cristallization before the high temperature region has been reached. At very low temperatures, the experimental and the calculated values of $C_{p}$ tend both to the classical Dulong-petit value expected for a harmonic solid $\left(C_{p} \approx 25 J K^{-1} 1 g^{-1}\right)$.

Note that the drop of calculated $C_{p}$ starts about $200 \mathrm{~K}$ above the value of $T_{g}$ determined from the bend of the enthalpy or of the volume. This indicates that $T_{g}$, 
evaluated from the change of slope, is an extrapolated quantity which characterizes a process (the GT) actually smeared out over a relatively wide temperature range. The drop of $C_{p, e x p}$ is, however, much sharper, i.e., the GT occurs across a narrower temperature range (of about $50 \mathrm{~K}$ ) in the experiments. The much larger smearing of the GT in our simulations is a consequence of the high transition temperature $T_{g}$, or equivalently, the high cooling rate used as argued by Angell et al. [68]: The temperature interval across which the relaxation time changes by a given factor decreases with decreasing temperature. This is due to the strong dependence of the relaxation time on temperature, e.g. Arrhenius or Vogel-Fulcher behavior (s. section (6.1)). In view of the higher $T_{g}$ for the binary system and the larger smearing of its GT, a comparison between $C_{p}$ data of the binary and the ternary system provides an additional support to Angell's argumentation.

At the end of this chapter we shall add a remark concerning the temperature dependence of the volume at high temperatures. For the ternary system as well as for the binary one, the volume exhibits a conspicuous kink well above the glass temperature $T_{g}$; around $1500 K$ for $\mathrm{Ni}_{25} \mathrm{Zr}_{60} \mathrm{Al}_{15}$ and around $1700 \mathrm{~K}$ for $\mathrm{Ni}_{40} \mathrm{Zr}_{60}$. This somewhat surprising feature cannot be attributed to a lack of equilibration time since in that temperature region the systems have typical relaxation times in the range of few picoseconds, whereas both systems have been equilibrated for at least one nanosecond before we start the evaluations. This effect will be related to other dynamical features exhibited by these systems in the same temperature range (s. section (7.2)). We note that Ohsaka et al. [69] also observed an anomaly in the specific volume of $\mathrm{NiZr}_{2}$ liquid alloy above its melting point. The measurements have been achieved by using levitation methods. 


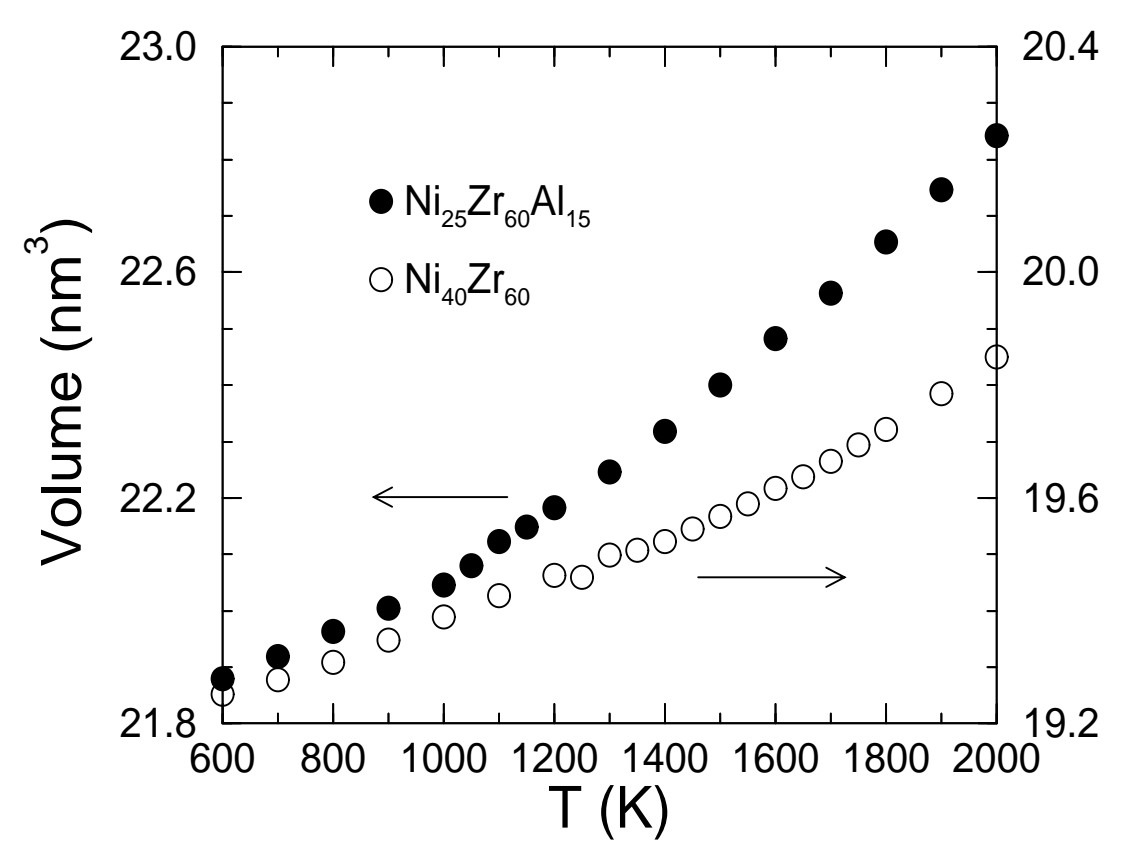

Figure 4.2: Volume $\mathrm{V}$ of the simulation cell versus temperature $\mathrm{T}$ for the ternary $\mathrm{Ni}_{25} \mathrm{Zr}_{60} \mathrm{Al}_{15}$ and binary $\mathrm{Ni}_{40} \mathrm{Zr}_{60}$ alloys.

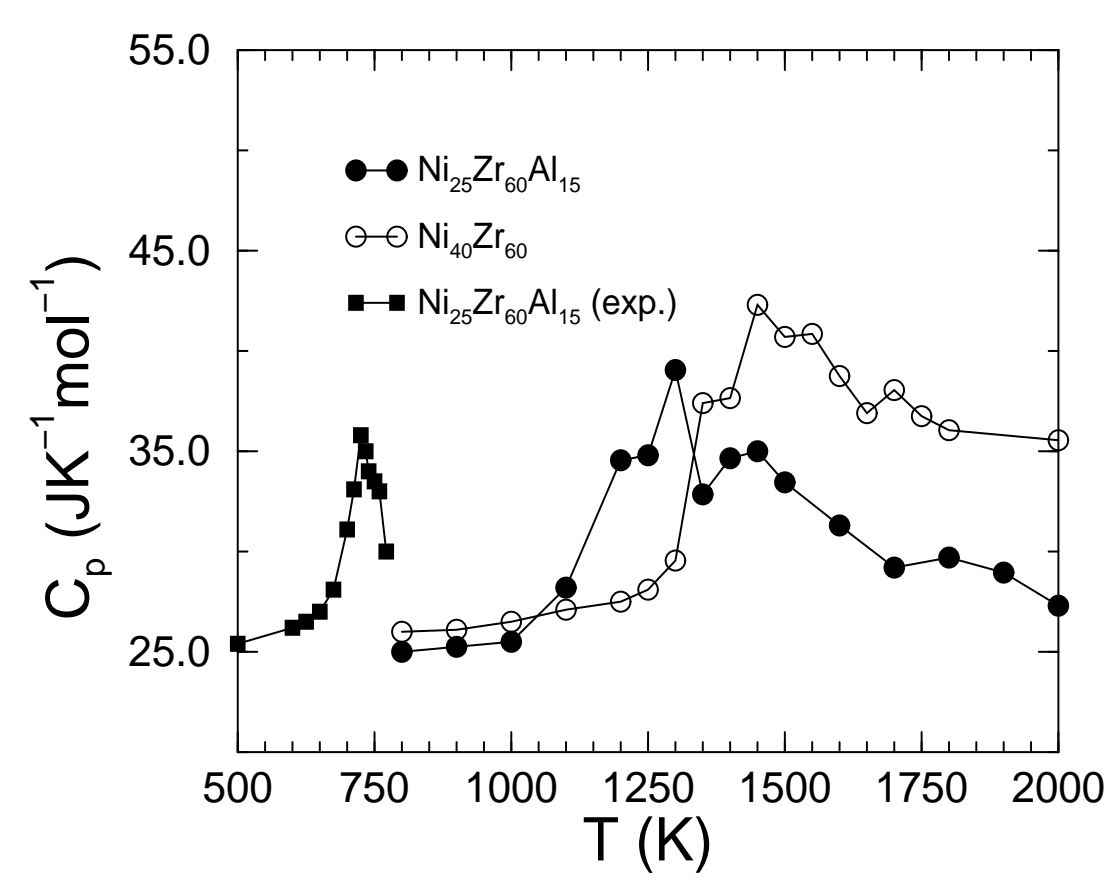

Figure 4.3: Specific heat $C p$ versus temperature $\mathrm{T}$ for the ternary $\mathrm{Ni}_{25} \mathrm{Zr}_{60} \mathrm{Al}_{15}$ and binary $\mathrm{Ni}_{40} \mathrm{Zr}_{60}$ alloys. The experimental results (full squares) are measurements of Inoue et al. 4]. 


\section{Chapter 5}

\section{Structural Properties}

In this section an analysis of the atomic structure is presented for the ternary system $\mathrm{Ni}_{25} \mathrm{Zr}_{60} \mathrm{Al}_{15}$. A comparison with the structure of the binary $\mathrm{Ni}_{40} \mathrm{Zr}_{60}$ will be made in order to understand which structural changes take place in the system when some of the $\mathrm{Ni}$ atoms are substituted with Al. For the structural characterization of binary $\mathrm{Ni}_{40} \mathrm{Zr}_{60}$, we use the results of the detailed investigation performed by Hausleitner et al. [57] concerning the atomic structure of amorphous binary Ni-Zr alloys using MD simulations and the NFE-TBB model, an investigation which contributed much to the fundamental understanding of the structure of transition-metal amorphous alloys.

\subsection{Pair distribution function}

The atomic arrangement in amorphous materials is conveniently described in terms of the pair distribution function (PDF) $g(r)$ [70, 71]. For an homogeneous system with an averaged number density $\rho_{0}, \rho_{0} g(r)$ gives the probability density that a particle is separated by a distance $r$ from another one. The PDF is obtained by means of a three-dimensional averaging procedure and retains only one-dimensional information. It contains the basic quantitative information which can be gained from diffraction experiments, like x-ray or neutron scattering.

There are some elementary conclusions, which can be drawn upon and from $g(r)$. Since the interatomic distances cannot be smaller than the atomic core diameters, we have $\lim _{r \rightarrow 0} g(r)=0$. For very large separations, $g(r)$ approaches unity and the system behaves as a structureless continum, $\lim _{r \rightarrow \infty} g(r)=1$.

The average number of neighbors of a given atom up to a distance $\mathrm{R}$ is given by

$$
4 \pi \rho_{0} \int_{0}^{R} r^{2} g(r) d r .
$$

In particular, we define the coordination number $z$, which gives the number of "nearest-neighbors", as the number of neighbors up to the first minimum of $g(r)$. 
An experimentally accessible quantity which is closely related to $g(r)$ is the coherent scattering intensity $I^{c o h}$. For one-component system the relation holds

$$
I^{c o h}(k)=N f^{2}+N f^{2} \rho_{0} \int 4 \pi r^{2}[g(r)-1] \frac{\sin (k r)}{k r} d r,
$$

where $k$ is the scattering wave vector, $f$ the atomic scattering factor, and $N$ the number of atoms in the sample. The static structure factor is defined by

$$
S(k)=\left(I^{c o h}(k) / N-f^{2}\right) / f^{2}=1+\rho_{0} \int 4 \pi r^{2}[g(r)-1] \frac{\sin (k r)}{k r} d r .
$$

Eq. 6.10 can be generalized to a two-component system as follows

$$
I^{c o h}(k)=N\left[c_{1} c_{2}\left(f_{1}-f_{2}\right)^{2}+\sum_{i, j=1}^{2} c_{i} c_{j} f_{i} f_{j} S_{i j}^{F Z}(k)\right],
$$

where $c_{i}$ and $f_{i}$ are the concentration and the atomic scattering factor, respectively, of component $i . S_{i j}^{F Z}(k)$ is the Faber-Ziman partial structure factor related to the partial PDF $g_{i j}(r)$ through

$$
S_{i j}^{F Z}(k)=1+\rho_{0} \int 4 \pi r^{2}\left[g_{i j}(r)-1\right] \frac{\sin (k r)}{k r} d r .
$$

Bhatia and Thornton have adopted a different approach in order to sub-divide the coherently scattered intensity

$$
I^{c o h}(k) / N=<f>^{2} S_{N N}(k)+\left(f_{1}-f_{2}\right)^{2} S_{c c}(k)+2<f>\left(f_{1}-f_{2}\right)^{2} S_{N c}(k),
$$

where $<>$ denote the average over the two components. $S_{N N}(k), S_{c c}(k)$ and $S_{N c}(k)$ are called the number-number, concentration-concentration and numberconcentration structure factors, respectively. They are given in terms of the partial PDFs as follows

$$
\begin{aligned}
S_{N N}(k) & =1+\rho_{0} \int 4 \pi r^{2}\left[g_{N N}(r)-1\right] \frac{\sin (k r)}{k r} d r \\
S_{N c}(k) & =\rho_{0} \int 4 \pi r^{2} g_{N c}(r) \frac{\sin (k r)}{k r} d r \\
S_{c c}(k) & =c_{1} c_{2}\left[1+\rho_{0} \int 4 \pi r^{2} g_{c c}(r) \frac{\sin (k r)}{k r} d r\right],
\end{aligned}
$$

where

$$
\begin{aligned}
g_{N N}(r) & =c_{1}^{2} g_{11}(r)+c_{2}^{2} g_{22}(r)+2 c_{1} c_{2} g_{12}(r), \\
g_{N c}(r) & =c_{1} c_{2}\left[c_{1} g_{11}(r)-c_{2} g_{22}(r)-\left(c_{1}-c_{2}\right) g_{12}(r)\right] \\
g_{c c}(r) & =c_{1} c_{2}\left[g_{11}(r)+g_{22}(r)-2 g_{12}(r)\right] .
\end{aligned}
$$


From Eq. (5.6) it follows that $S_{N N}(k)$ and $g_{N N}(r)$ represent an average structure without regard to the type of atom concerned. Thus the number-number correlation functions correspond to the topological distribution of atoms of components 1 and 2 . On the other hand, the concentration-concentration correlation functions $S_{c c}(k)$ and $g_{c c}(r)$ are related to the ordering or clustering tendency in the alloy, which makes them suitable for describing the chemical order in the binary alloy.

\section{$5.2 \quad$ Structural models of glasses}

Bernal [72] was the first who proposed a structural model for monoatomic liquids based upon a dense random packing of hard spheres (DRPHS), which was extended later to amorphous metallic solids. This model was found to reproduce even the second peak splitting in the radial distribution function, which is observed as a structural feature common to many metallic glasses. In view of this success, Polk [73] extended the DRPHS model to include the binary amorphous system. He suggested that metalloid atoms could occupy the Bernal holes in the DRP structure of the metal atoms. Polk's model turned out to be rather successful for transition metal-metalloid (TM) glasses. Provided the metalloid atoms preferentially occupy trigonal prismatic holes of the DRP formed by the transition-metal atoms, the local environment of the atoms is similar to that observed in the crystalline phases. However, in the DRP structure, the number of holes having an appropriate size to accomodate all of the metalloid atoms was found too small in the typical glass-forming range concentration of these alloys.

An alternative model was proposed by Gaskell [74], called the stereochemically defined model. This model suggets that the glass is made up of local structural units which may also be present in the crystalline structure. For example, crystalline borides, phosphorides, silicides, etc., of those transition metals which readily form glasses, have structures based on trigonal prismatic coordination of the metalloid by the transition-metal atoms. This structure persists over a wide range of compositions and over a range of radii ratios which differ widely from the ideal ratio of an undistorted trigonal prism, which suggets that this type of packing is particularly stable. According to the stereochemically defined model, in modelling the structure of the glassy phase of these TM alloys, one should proceed by a random packing of such trigonal-prismatic units rather than of individual atoms (see Fig. (5.1). Differences in structure with concentration are described by variations in the way these structural units are arranged.

More recently Hausleitner et al. [57] have established the validity of the stereochemically defined model for a series of intertransition-metal glasses using atomistic simulations based on the quantum mechanically derived NFE-TBB model (see chapter 3). They demonstrated a clear trend from trigonal prismatic to polytetrahedral local order in the serie $\mathrm{Ni}-\mathrm{Y}, \mathrm{Ni}-\mathrm{Zr}$ and $\mathrm{Ni}-\mathrm{Nb}$ and within a given system with in- 
creasing Ni content, a topological trend which is also found in the corresponding crystalline phases.

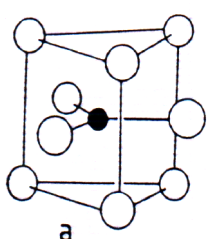

a

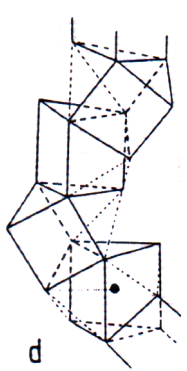

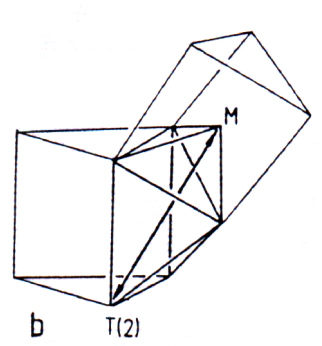

b

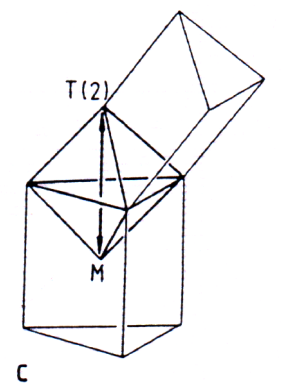

Figure 5.1: (a) Trigonal prism with capping atoms. (b) Two trigonal prisms (TP) with a common edge linked as in the cementite structure.

(c) TPs with the edge-sharing arrangement observed in $\mathrm{Fe}_{3} \mathrm{P}$ and $\mathrm{Ti}_{3} \mathrm{P}$ lattices. (d) Disordered chain of TPs produced by random edge-sharing [125].

\subsection{Comparison with experimental results}

The atomic structure of $\mathrm{Ni}_{25} \mathrm{Zr}_{60} \mathrm{Al}_{15}$ amorphous alloy was investigated exprimentally by Matsubara et al. [75], who used anomalous X-ray scattering (AXS) in combination with the ordinary X-ray diffraction to determine the ordinary and the environmental radial distribution functions (RDF). The results of these measurement are reproduced in Fig. (5.2). To check the validity of our model, we show in this figure also the calculated RDF from our MD simulation.

The curves at the top of Fig. (5.2) correspond to the ordinary RDFs which represents the radial distribution around the average atom, independent of the atom species. It is the weighted sum of the six partial pair correlation functions $g_{i j}(r)$

$$
4 \pi r^{2} \rho(r)=4 \pi r^{2} \sum_{i, j} \sum_{=Z r, N i, A l} \frac{c_{i} f_{i} f_{j}}{<f>^{2}} g_{i j}(r),
$$

with $<f>=\sum_{i} c_{i} f_{i} . \quad c_{i}$ and $f_{i}$ are the atomic concentration and the atomic scattering factor of the element $i$ respectively. 


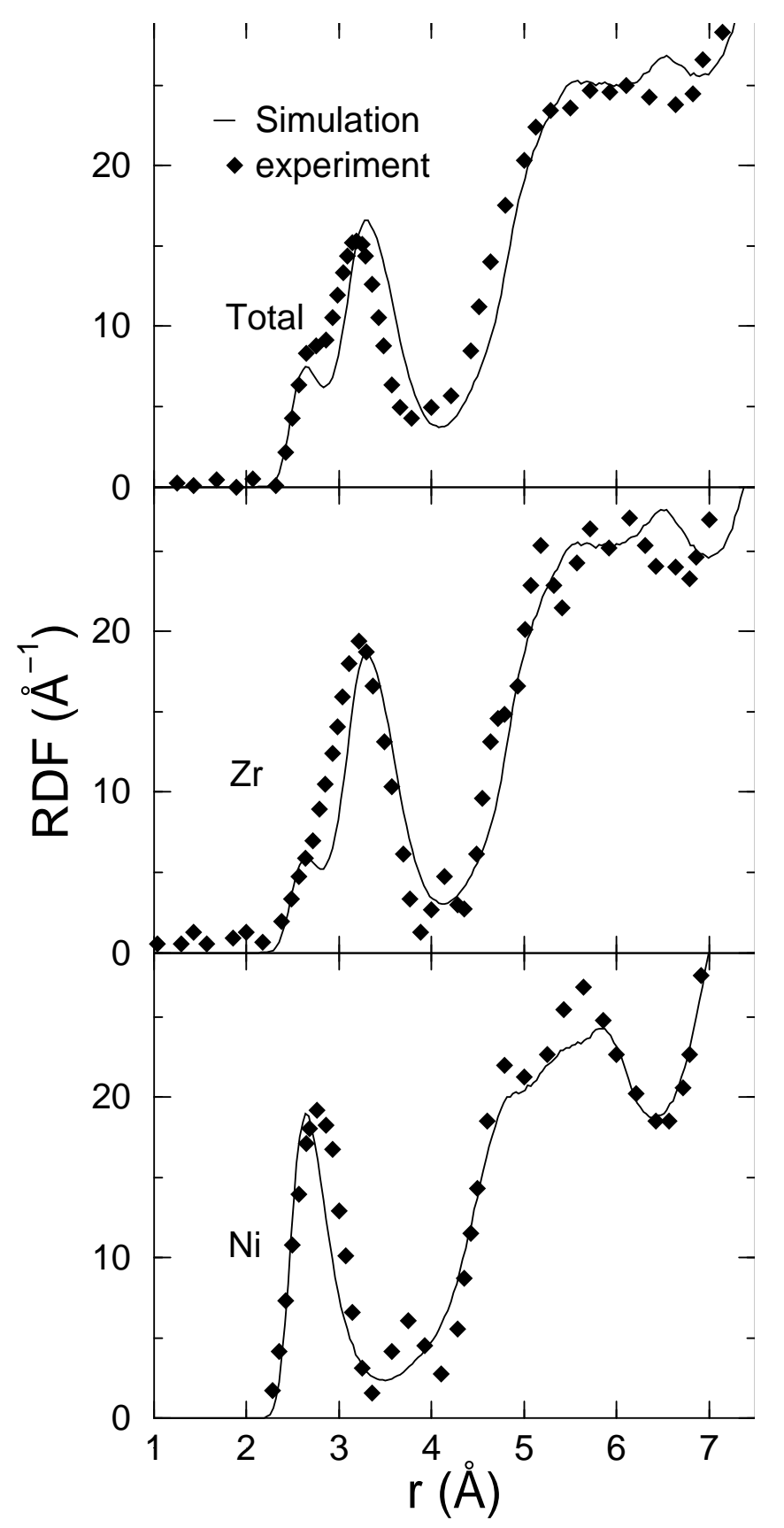

Figure 5.2: The ordinary (top) and environmental RDFs for Zr (centre) and $\mathrm{Ni}$ (bottom) of $\mathrm{Ni}_{25} \mathrm{Zr}_{60} \mathrm{Al}_{15}$ amorphous alloy at $300 \mathrm{~K}$. Calculated (solid line) and measured (filled diamond) data are compared. The experimental results are from Matsubara et al. [75]. 
The curves at the centre and the bottom of Fig. (5.2) correspond to the environmental RDFs for $\mathrm{Zr}$ and $\mathrm{Ni}$, respectively. The experimental curves have been determined by the AXS method. The environmental RDF provides the weighted average over all atomic pairs around a given element A

$$
\begin{gathered}
4 \pi r^{2} \rho_{A}(r)=4 \pi r^{2} \sum_{i=Z r, N i, A l} \frac{\Re\left[f_{i}\left(E_{1}\right)+f_{i}\left(E_{2}\right)\right]}{W\left(E_{1}, E_{2}\right)} g_{A i}(r), \\
W\left(E_{1}, E_{2}\right)=\sum_{i} c_{i} \Re\left[f_{i}\left(E_{1}\right)+f_{i}\left(E_{2}\right)\right]
\end{gathered}
$$

where $f_{i}$ is the total X-ray atomic scattering factor, a complex number which contains an anomalous dispersion term in addition to the usual atomic scattering factor. The measurements have been carried out at two energies $E_{1}$ and $E_{2}$ below the absorption edge of the considered atom. $\Re$ denotes the real part of the values in the brackets. For more details we refer to reference [75].

Fig. (5.2) demonstrates that qualitatively as well as quantatively the experimental and the calculated curves agree well. The height and the location of the peaks are well reproduced. The peaks positions, as given in tab.(5.1), are found to be within $4 \%$ of the values obtained bei Matsubara et al.. However, the splitting of the first peak in the calculated $\mathrm{RDF}$ of $\mathrm{Zr}$ is somewhat exagerated relatively to the measured one, where the latter shows only a weak hump at the same location (note that this hump becomes more and more important with annealing and cristallization as shown in [75]). The hump represents the Ni-Zr correlation whereas the main peak results from $\mathrm{Zr}-\mathrm{Zr}$ correlations. The discrepancy is due to the fact that our model underestimates the Ni-Zr interatomic distance and simultaneously overestimates the Zr-Zr one, as can be seen from tab.(5.1).

\subsection{Bond-angle distribution}

To take a first insight in the topological short-range order (SRO) present in the structure of $\mathrm{Ni}_{25} \mathrm{Zr}_{60} \mathrm{Al}_{15}$, we consider in this section the distribution function of the bond angles around each atom species. This triple-correlation function is defined as an average of bond angles between a reference atom and the pairs of atoms within a radius $R_{\max }$, chosen here as the first minimum of the total radial correlation function $g_{\text {tot }}(r)\left(R_{\max } \approx 4.1 \AA\right)$. The results of this analysis are displayed in Fig. (5.3).

The distributions of the angles formed by bonds centered at the $\mathrm{Zr}$ and Ni sites present the same features as those calculated by Hausleitner et al. [57] for the binary systems $\mathrm{Ni}_{50} \mathrm{Zr}_{50}$ and $\mathrm{Ni}_{33} \mathrm{Zr}_{67}$. In [57] it is pointed out that the peaks in these angular correlations are compatible with a local trigonal prismatic order similar to that in the cristalline $\mathrm{CrB}$ structure. Patterns of other trigonal prismatic structures, like $\mathrm{Fe}_{3} \mathrm{C}$, are also detected. In CrB-type compounds, like NiZr, the trigonal prisms 
form layers with $\mathrm{B}(\mathrm{Ni})$ atoms centering the prisms. The $\mathrm{B}(\mathrm{Ni})$ atoms being arranged in zigzag chains within this configuration.

Interpreting the here deduced bond angle distributions in the light of the picture advanced by Hausleitner et al., the bond angles centered at $\mathrm{Zr}$ sites correspond to those on the triangular and square faces of a prism $\left(\theta \approx 60^{\circ} ;, \theta \approx 90-100^{\circ}\right)$ and to the rotation of two edge-sharing prisms $\left(\theta \approx 145^{\circ}\right)$ (like in a $\mathrm{Fe}_{3} \mathrm{C}$ structure). The bond angles centered at Ni sites (fig), $\theta \approx 75^{\circ}$ and $\theta \approx 135^{\circ}$, correspond to angular correlations between a $\mathrm{Ni}$ atom centering the prism and two other atoms on the vertices. The vertices being occupied in this model by $\mathrm{Zr}$ atom predominatly. The pronounced peak at $\theta \approx 110^{\circ}$ in the $\mathrm{Ni}-\mathrm{Ni}-\mathrm{Ni}$ angular correlation is very close to the chain angle of $\theta=110^{\circ}$ in the $\mathrm{CrB}$ structure.

The distribution of the bond angles at $\mathrm{Al}$ sites shows a different pattern than those for $\mathrm{Zr}$ or $\mathrm{Ni}$ sites. It exhibits well-defined peaks at $\theta \approx 60^{\circ}, \theta \approx 120^{\circ}$ and $\theta \approx 180^{\circ}$, which are very close to the icosahedral bond angles $\left(\theta \approx 63.5^{\circ}, \theta \approx 116.5^{\circ}\right.$ and $\theta \approx 180^{\circ}$ ). This suggests that $\mathrm{Al}$ atoms occupy sites with a predominatly icosahedral symmetry, in contrast to $\mathrm{Zr}$ and $\mathrm{Ni}$ atoms which occupy sites with a trigonal prismatic symmetry. Conclusions which can be drawn from this observation will be discussed in the next section.

\subsection{Radial distribution functions}

\subsubsection{Partial PDFs}

Fig. (5.4) shows the calculated partial pair correlation functions $g_{i j}(r)$ in the liquid phase $(T=2000 K)$ and in the glassy state $(T=300 K)$. For purpose of clarity we show the correlation functions associated with each pair of atoms separately.

$g_{N i N i}(r)$ exhibits only a very weak nearest-neighbour peak, which becomes smaller with decreasing temperature. The first peak in $g_{N i Z r}(r)$ is very intense, even more than in the $\mathrm{Zr}-\mathrm{Zr}$ distribution. This manifests the tendency for $\mathrm{Ni}$ atoms to be preferentially surrounded by $\mathrm{Zr}$ as nearest-neighbors. Such a short-range order between $\mathrm{Ni}$ and $\mathrm{Zr}$ atoms has been already observed experimentally [76, 64, and confirmed theoretically [57, 77] in binary Ni-Zr alloys, and is attributed to the pronounced non-addivity character of the pair potentials as argued by Hausleitner et al. [57] (see section 3.4 ). In the subsystem $\mathrm{Zr}-\mathrm{Al}$, the pronounced first peak and first minimum in $g_{A l A l}(r)$ deserves particular attention. It is rather unexpected in the light of the small $\mathrm{Al}$ concentration used here and the good mixing behavior of the binary Zr-Al system.

As discussed in Section 3.4, the three binary systems Ni-Zi, Ni-Al and $\mathrm{Zr}-\mathrm{Al}$ manifest good compound-forming behaviour. It is obvious that the ultimate structure of the ternary system $\mathrm{Ni}_{25} \mathrm{Zr}_{60} \mathrm{Al}_{15}$ is determined by the competition of the mixing ability of the three binary subsystems. The bonding strength of the two mi- 

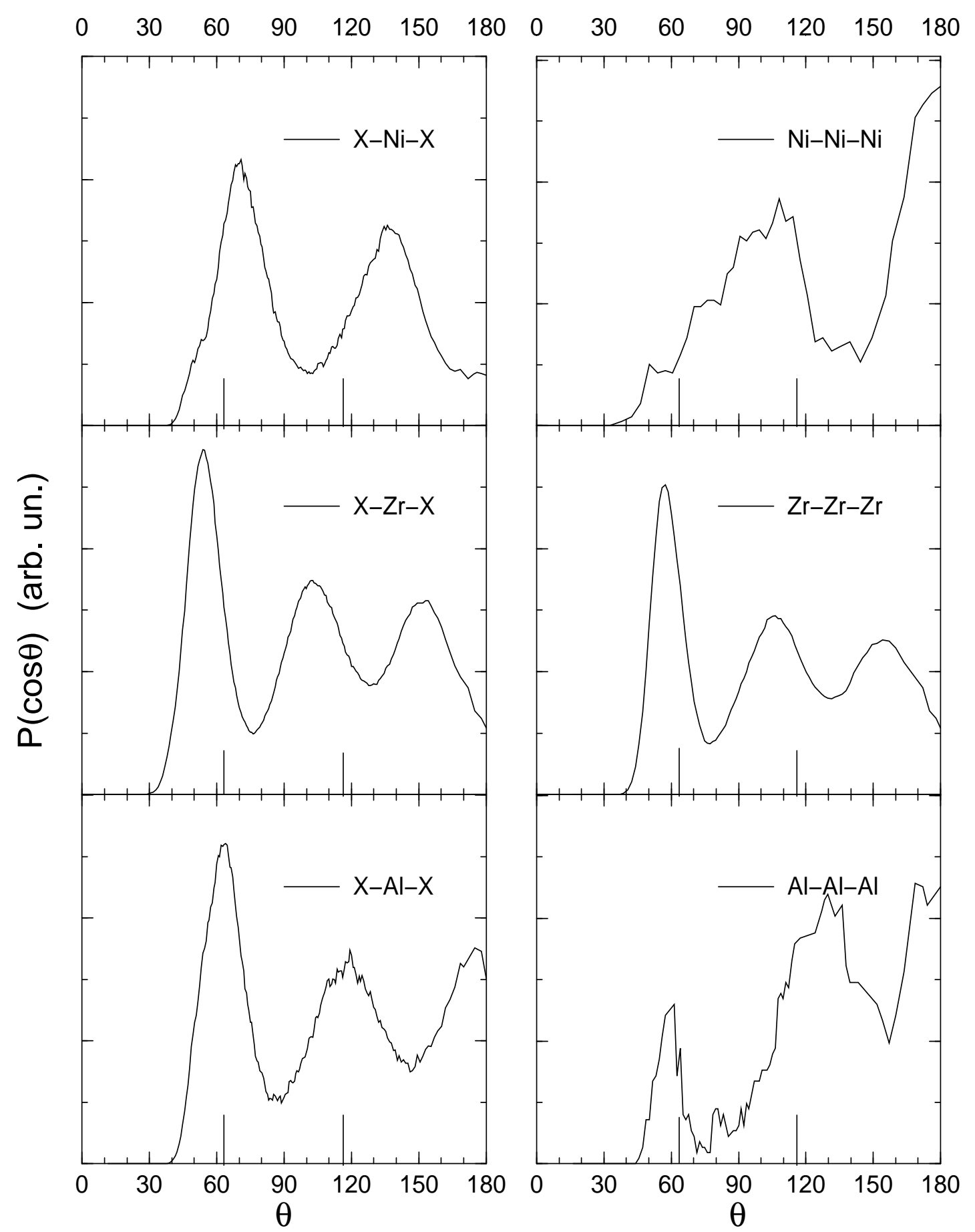

Figure 5.3: Partial bond-angle distributions in amorphous $\mathrm{Ni}_{25} \mathrm{Zr}_{60} \mathrm{Al}_{15}$ alloy. $\mathrm{X}$ means that all atom species are considered. The vertical lines indicate the bond angles in a regular icosahedron $\left(\theta=63.5^{\circ}, 116.5^{\circ}, 180^{\circ}\right)$. 


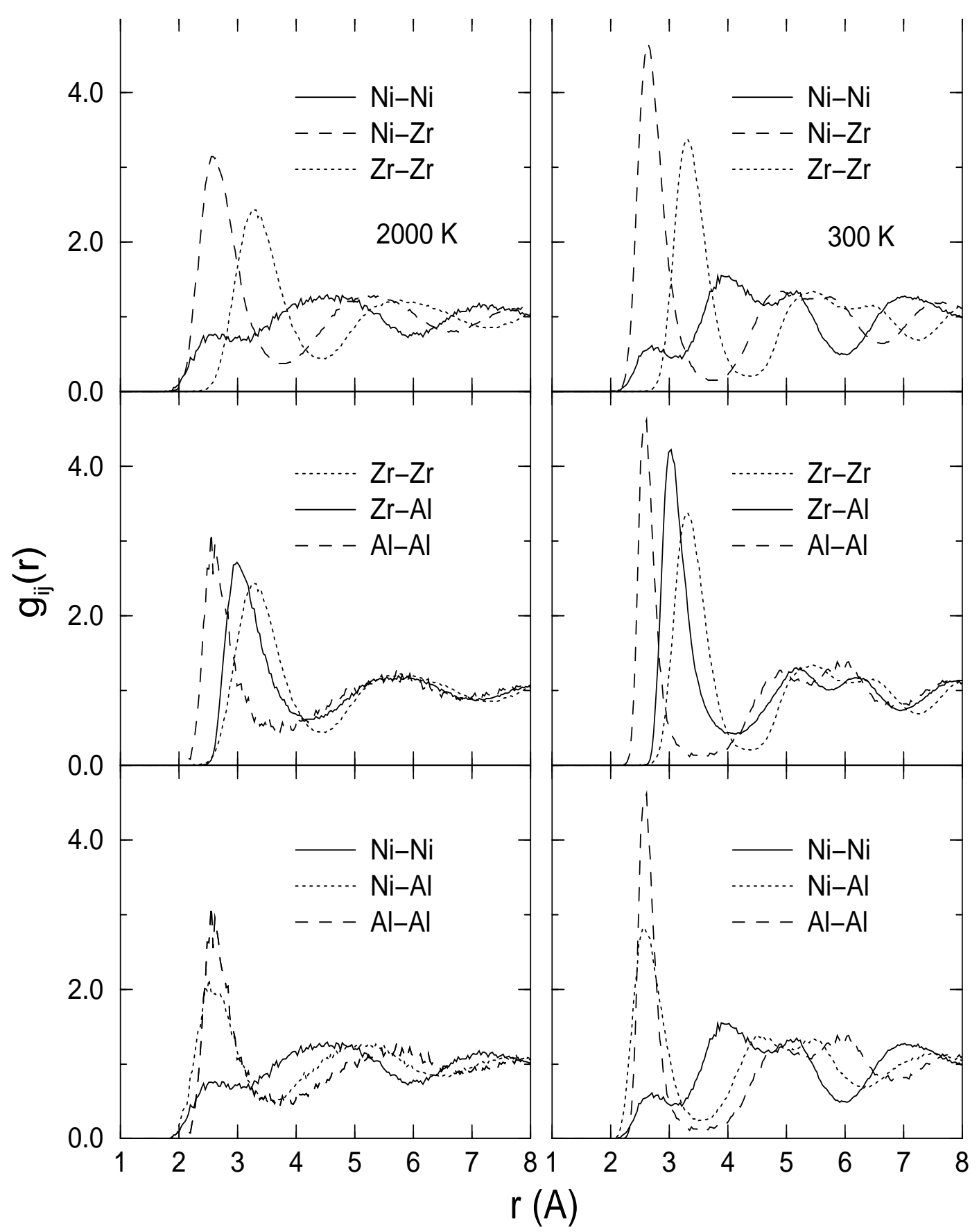

Figure 5.4: Partial pair correlation functions $g_{i j}(r)$ of $\mathrm{Ni}_{25} \mathrm{Zr}_{60} \mathrm{Al}_{15}$ at $T=$ $2000 K$ and $T=300 K$. For clarity, the $g_{i j}(r)$ s associated with the three subsets $\mathrm{Ni}-\mathrm{Zr}, \mathrm{Ni}-\mathrm{Al}$ and $\mathrm{Zr}-\mathrm{Al}$ are represented separately. 
nority species $\mathrm{Ni}$ and $\mathrm{Al}$ to the majority species $\mathrm{Zr}$ is of particular importance here. Since the compound-forming tendency of Ni-Zr seems to be stronger than that of $\mathrm{Al}-\mathrm{Zr}$, as seen in section 3.4, it is expected that Al-Zr heterocoordination preference will be depressed by the stronger Ni-Zr preference. From that, an Al-Al clustering tendency may occur. This can explain the pronounced first peak in the Al-Al distribution function. Moreover, the atom-size effect may be an additionnal cause for this clustering feature. By their smaller size, $\mathrm{Ni}$ atoms are more appropriate than $\mathrm{Al}$ atoms to match in the Bernal holes of the $\mathrm{Zr}$ matrix. The total demixing of $\mathrm{Al}$ atoms is, nevertheless, hindered by the moderate $\mathrm{Al}-\mathrm{Zr}$ and strong $\mathrm{Ni}-\mathrm{Al}$ heterocoordination, as show the pronounced nearest-neighbors peaks in $g_{N i A l}(r)$ and $g_{Z r A l}(r)$. The weak clustering tendency of $\mathrm{Al}$ atoms can be clearly observed on the snapshot of Fig. (5.5). A tendency to form chain-like structures of Al may be identified, in addition.

Table 5.1: nearest-neighbor distances $d_{i j}$ and coordination numbers $z_{i j}$ in amorphous $\mathrm{Ni}_{25} \mathrm{Zr}_{60} \mathrm{Al}_{15}$. The experimental results are from measurements of Matsubara et al. [75].

\begin{tabular}{lcccc}
\hline \hline & $d_{i j}[\AA]($ calc. $)$ & $d_{i j}[\AA]($ exp. $)$ & $z_{i j}$ (calc.) & $z_{i j}(\exp )$. \\
\hline $\mathrm{Ni}-\mathrm{Ni}$ & 2.65 & & 0.50 & \\
$\mathrm{Zr}-\mathrm{Ni}$ & 2.63 & 2.67 & 3.02 & 2.2 \\
$\mathrm{Ni}-\mathrm{Zr}$ & 2.63 & 2.67 & 7.25 & \\
$\mathrm{Zr}-\mathrm{Zr}$ & 3.30 & 3.17 & 9.93 & 10.3 \\
$\mathrm{Ni}-\mathrm{Al}$ & 2.60 & & 1.20 & \\
$\mathrm{Al}-\mathrm{Ni}$ & 2.60 & & 2.00 & \\
$\mathrm{Zr}-\mathrm{Al}$ & 3.02 & & 2.25 & 0.0 \\
$\mathrm{Al}-\mathrm{Zr}$ & 3.02 & & 8.99 & \\
$\mathrm{Al}-\mathrm{Al}$ & 2.59 & & 1.06 & \\
\hline \hline
\end{tabular}

The above given structural analysis suggests that the structure of the ternary alloy consists of a network of Ni-Zr units, where isolated $\mathrm{Ni}$ atoms are surrounded by $\mathrm{Zr}$ atoms, interpenetrated by $\mathrm{Al}$ groupings. According to the angle-distribution analysis in the last section, the Ni-Zr units have a trigonal prismatic topology, similar to that in the binary $\mathrm{Ni}-\mathrm{Zr}$ alloys, where a $\mathrm{Ni}$ atom centers the prism and the vertecis are occupied by Zr atoms.

\subsubsection{Structural comparison with binary $\mathrm{Ni}_{40} \mathrm{Zr}_{60}$}

In Fig. (5.6) we compare the PDFs of the binary compound $\mathrm{Ni}_{40} \mathrm{Zr}_{60}$ with those of the ternary $\mathrm{Ni}_{25} \mathrm{Zr}_{60} \mathrm{Al}_{15}$. The latter is obtained by substituting partially $\mathrm{Ni}$ with 


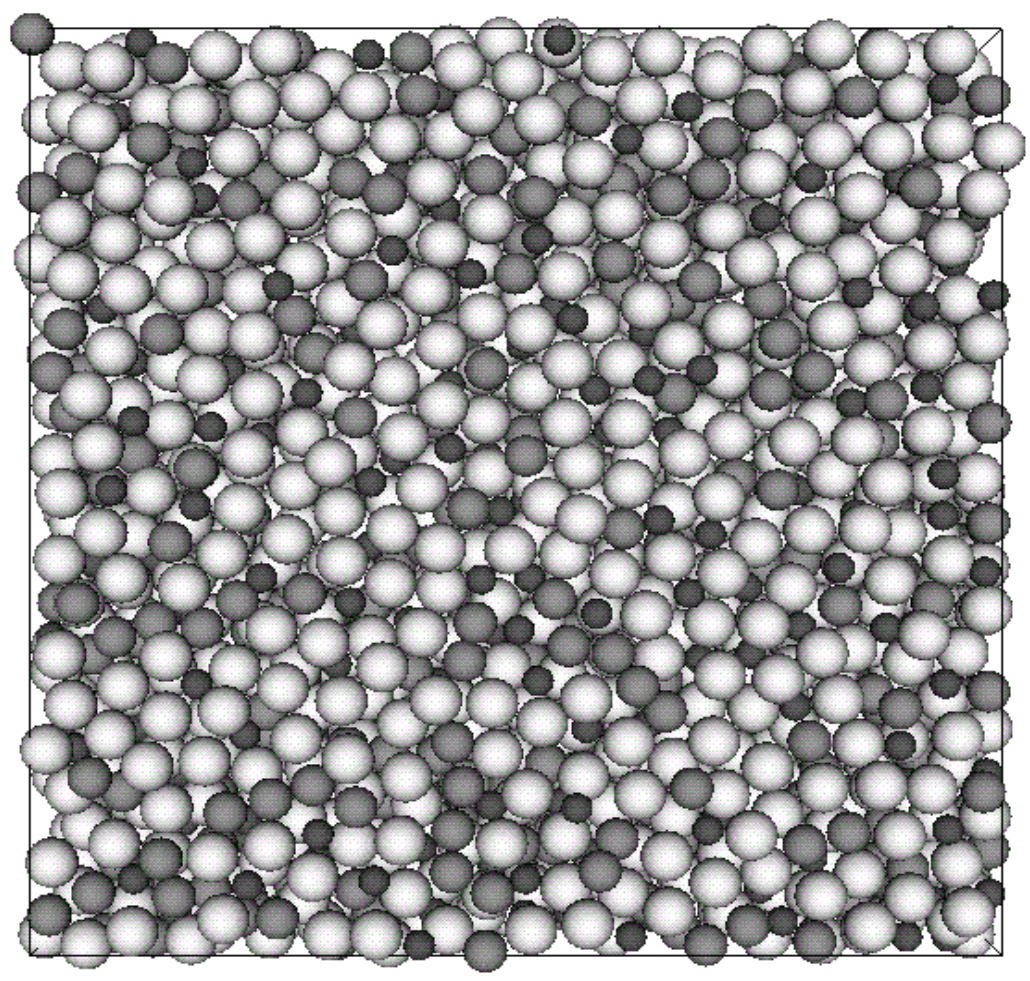

Figure 5.5: Cross section of an amorphous $\mathrm{Ni}_{20} \mathrm{Zr}_{60} \mathrm{Al}_{20}$ configuration at 800 $K$. The biggest spheres (light) are the Zr atoms, the smallest ones (dark) are the Ni atoms, and the middle spheres (grey) are the $\mathrm{Al}$ atoms. The sample contains 9216 particles. The box has a side length of $\approx 60 \AA$. In order to compare the structural behavior of $\mathrm{Ni}$ and $\mathrm{Al}$ atoms, we have chosen the same atom number for the two species. Notice that a $\mathrm{Ni}$ atom rarely has another $\mathrm{Ni}$ atom as nearest-neighbor. Whereas a weak clustering tendency of $\mathrm{Al}$ atoms can be clearly observed. Notice also the tendency to form Chain-like structures of $\mathrm{Al}$. 


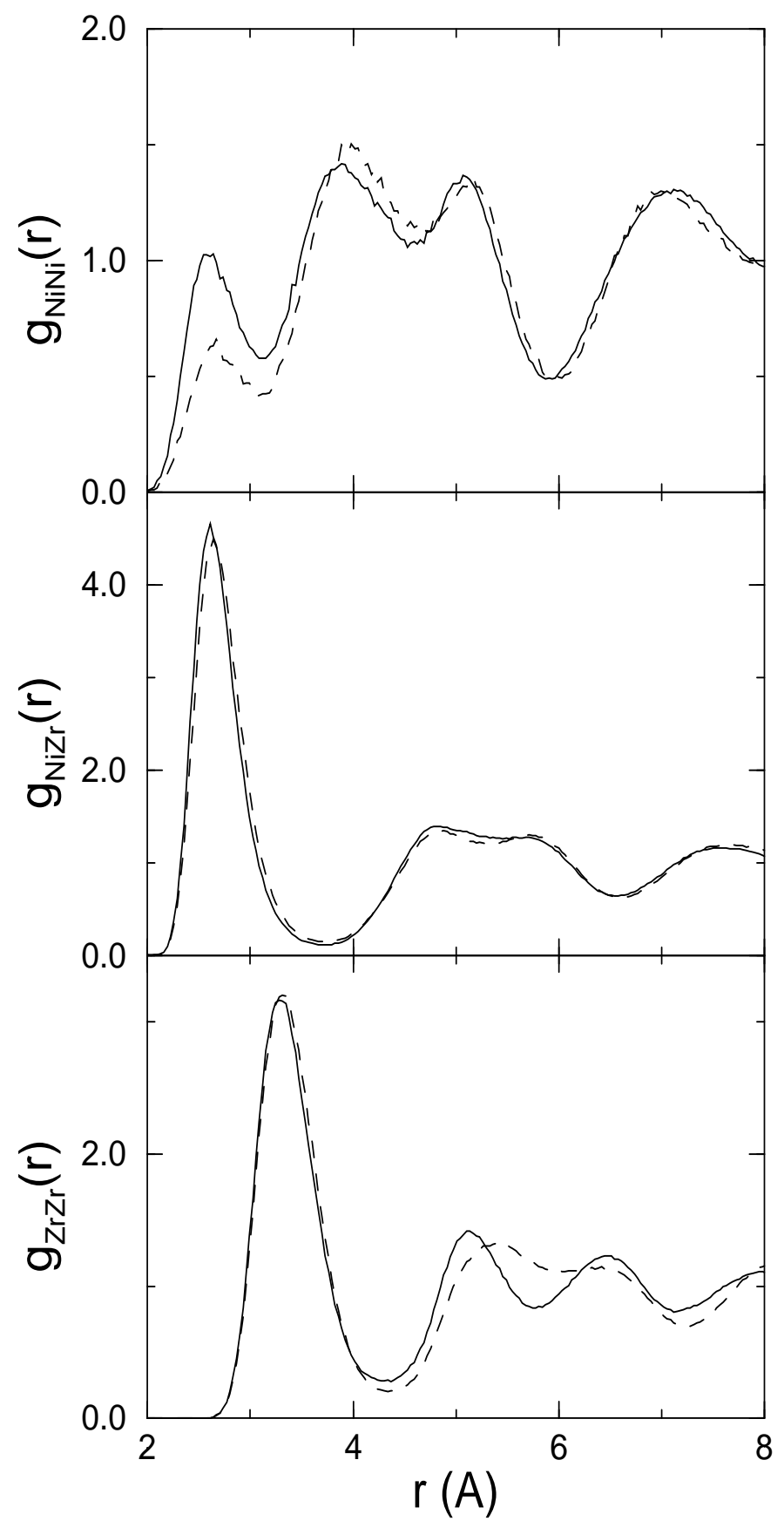

Figure 5.6: Partial pair correlation functions $g_{i j}(r)$ of the $\mathrm{Ni}_{25} \mathrm{Zr}_{60} \mathrm{Al}_{15}$ (dashed line) and $\mathrm{Ni}_{40} \mathrm{Zr}_{60}$ (solid line) amorphous alloys at $T=$ $300 \mathrm{~K}$. 
$\mathrm{Al}$ atoms, the $\mathrm{Zr}$ concentration being kept unchanged. We observe that $g_{N i N i}(r)$ exhibits a decrease of the nearest-neighbor peak, which is plausible because with decreasing $\mathrm{Ni}$ concentration the probability for a $\mathrm{Ni}-\mathrm{Ni}$ contact becomes smaller. The second peak, which marks the average distance between two cells centered by $\mathrm{Ni}$ atoms (within the suggested trigonal prismatic topology) has however grown slightly. By reducing the $\mathrm{Ni}$ concentration relatively to $\mathrm{Zr}$ atoms we create a surplus of $\mathrm{Zr}$ atoms which undergo a chemical bonding with $\mathrm{Al}$ atoms. The underlying topology of this bonding is of icosahedral type according to section 5.4. This can explain the change in the second shoulder of $g_{Z r Z r}(r)$ with $\mathrm{Al}$ alloying. More conspicuous is the invariance of the $\mathrm{Ni}-\mathrm{Zr}$ correlation up to a distance of more than twice the nearest-neighbor distance. These observations provide support to the above suggested picture of a ternary structure consisting of Ni-Zr units similar to those in the binary NiZr compound and surrounded by $\mathrm{Al}$ groupings.

\subsubsection{On the thermal stability of amorphous $\mathrm{Ni}_{25} \mathrm{Zr}_{60} \mathrm{Al}_{15}$}

The tendency to clustering of $\mathrm{Al}$ atoms may be related to the observations of Matsubara et al. [75], who found that the fraction of $\mathrm{Al}$ atoms around $\mathrm{Zr}$ in a as-quenched sample of amorphous $\mathrm{Ni}_{25} \mathrm{Zr}_{60} \mathrm{Al}_{15}$ was extremely reduced and that this fraction exhibits a drastic increase in the crystallized sample. Furthermore, it was found that the fraction of $\mathrm{Ni}$ atoms around $\mathrm{Zr}$ is almost unchanged with crystallization while the fraction of $\mathrm{Zr}$ around $\mathrm{Zr}$ atoms decreases markedly. The authors argued that the $\mathrm{Al}$ atoms must be rearranged around $\mathrm{Zr}$ for crystallization, which may retard the crystallization process in this system and explain thereby the effectiveness of $\mathrm{Al}$ addition in Ni-Zr binary system to thermally stabilize the Ni-Zr-Al amorphous alloys. Matsubara et al. showed that other multicomponent amorphous alloys with an extremely large supercooled liquid region exhibit similar feature, i.e. a pronounced change in the atomic arrangement between the amorphous and the crystallized state.

\subsection{Static Structure Factors and Short Range Or- der}

In this section we present the Faber-Ziman (FZ) and the Bathia-Thornton (BT) static structure factors. The partial FZ structure factor is defined as the Fourier transform of the partial PDF, analogous to Eq. (5.5). In the lack of a systematic definition of the BT structure factors for a ternary system, we restrict ourselves to the three binary subsystems $\mathrm{Ni}-\mathrm{Zr}, \mathrm{Zr}-\mathrm{Al}$, and $\mathrm{Ni}-\mathrm{Al}$, for which we determine the corresponding BT structure factors according to Eq. 6.17). The relative atomic concentrations are used in the corresponding formula. We attempt with this approach, albeit not exact, to obtain informations about possible concentration or 


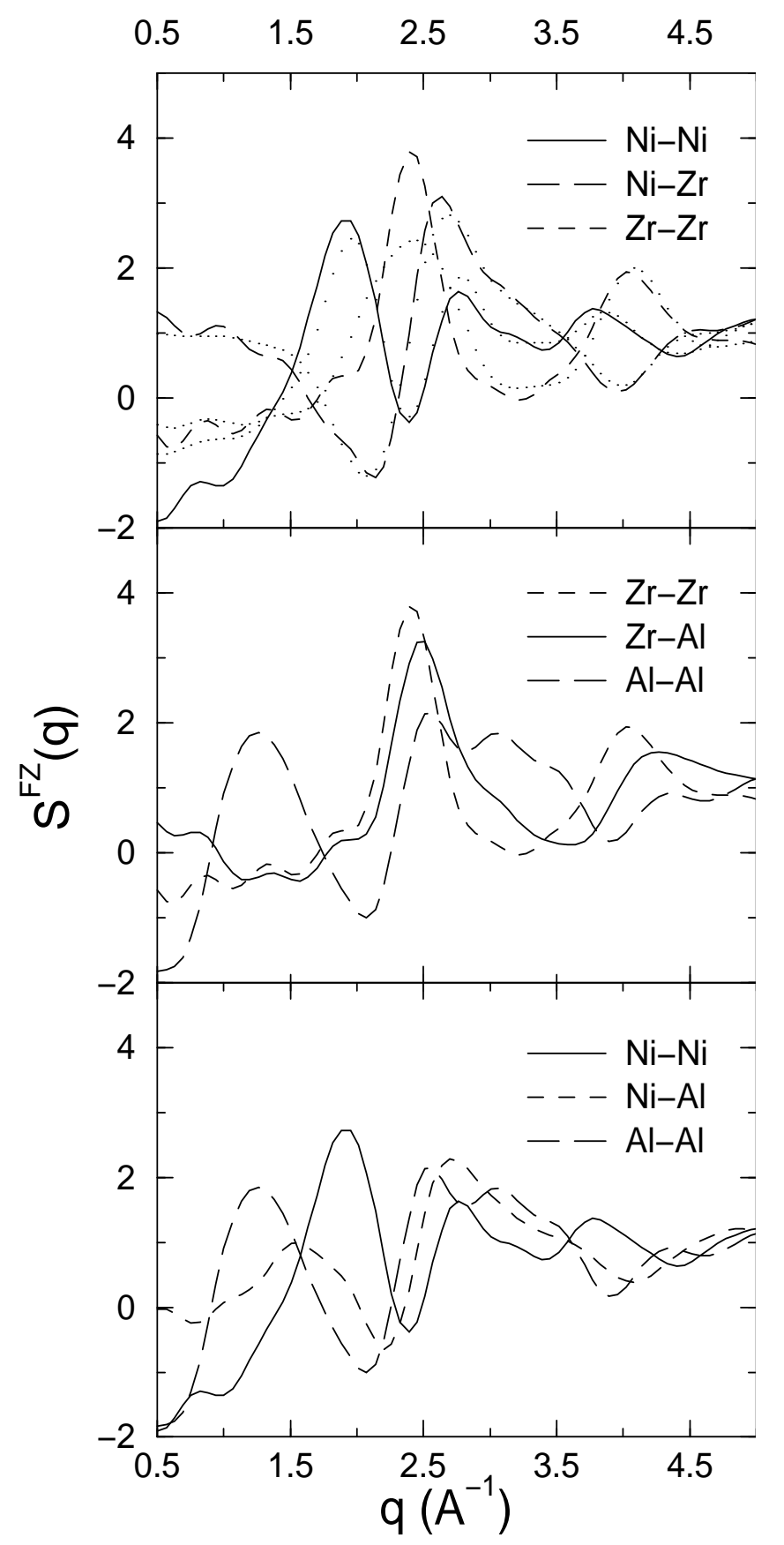

Figure 5.7: Faber-Ziman structure factors $S_{i j}^{F Z}(q)$ of $\mathrm{Ni}_{25} \mathrm{Zr}_{60} \mathrm{Al}_{15}$ at $T=$ $300 K$. For clarity, the $S_{i j}^{F Z}(q)$ s associated with the three subsets $\mathrm{Ni}-\mathrm{Zr}, \mathrm{Ni}-\mathrm{Al}$, and $\mathrm{Zr}-\mathrm{Al}$ are represented. For comparison, the FZ structure factors of the binary $\mathrm{Ni}_{40} \mathrm{Zr}_{60}$ alloy are given in dotted lines in the upper figure. 


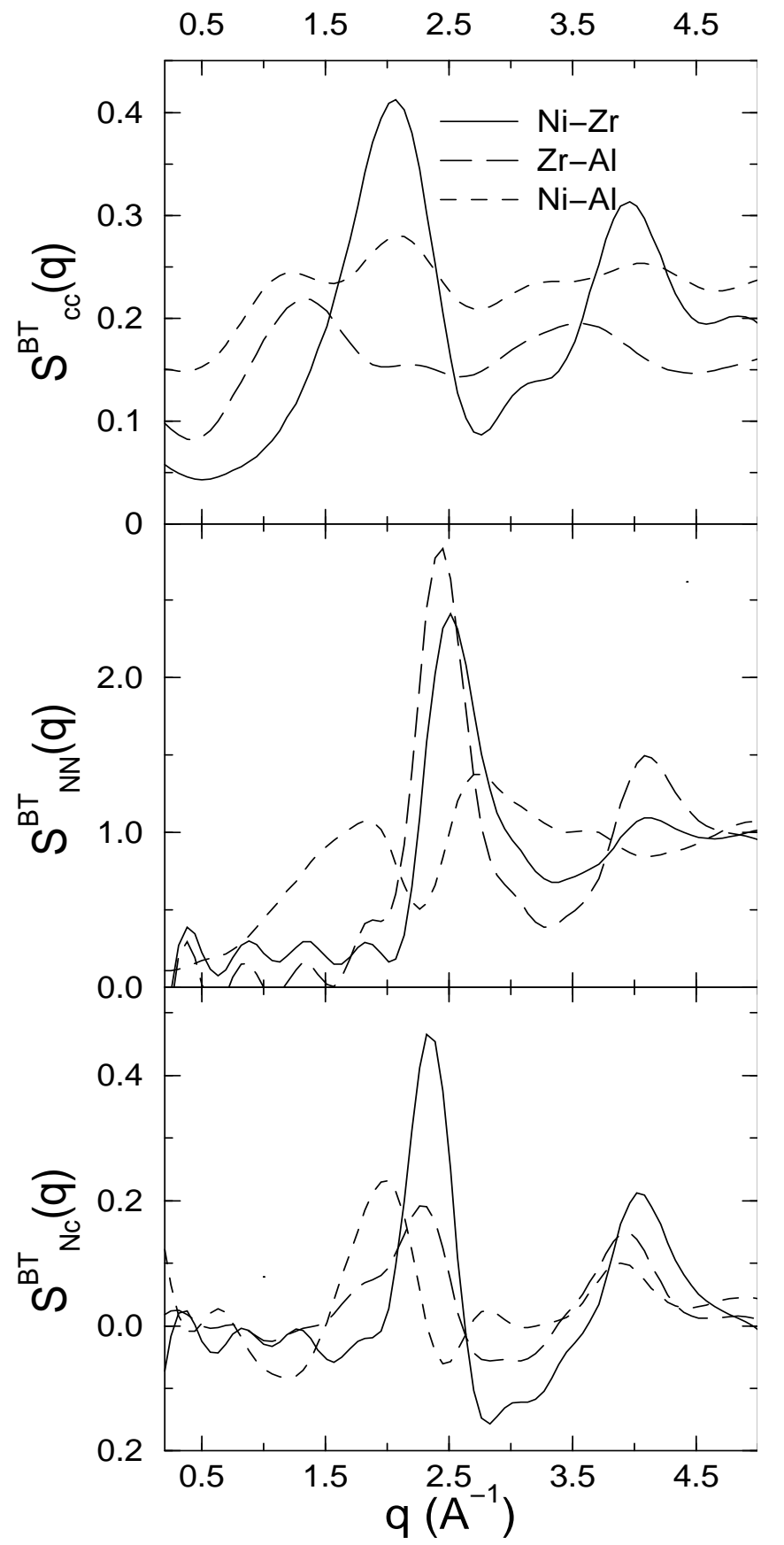

Figure 5.8: Bathia-Thornton structure factors $S^{B T}(q)$ of $\mathrm{Ni}_{25} \mathrm{Zr}_{60} \mathrm{Al}_{15}$ alloy at $T=300 K$. The $S^{B T}(q)$ s are calculated for the three subsets $\mathrm{Ni}-\mathrm{Zr}$ (solid line), Ni-Al (dashed line), and $\mathrm{Zr}-\mathrm{Al}$ (long-dashed) after Eq. (6.17) established for binary systems. 
density fluctuations in the ternary system, i.e. chemical or topological short range order, respectively.

In Fig. (5.7) we show the partial FZ structure factors $S_{i j}^{F Z}(q)$ for the subsystem Ni-Zr. We display additionally the results of the binary amorphous system $\mathrm{Ni}_{40} \mathrm{Zr}_{60}$ with dotted lines. On the whole, the two sets of $S_{i j}^{F Z}(q)$ are very similar, except that the main peak of $S_{Z r Z r}^{F Z}(q)$ is broader in the case of the binary system. $S_{N i N i}^{F Z}(q)$ differs from the two other structure factors by the appearance of a well defined prepeak at the left of the "main" peak, corresponding to the nearest-neighbor peak in $g_{N i N i}(r)$. The prepeak at the wave vector $q \approx 1.9 \AA^{-1}$ seems to result from a correlation on a length scale of about $3.3 \AA$, after the conventional relation, $l=2 \pi / q$, used to convert wave vectors in lengths. The main peak, at the wave vector $q \approx 2.8 \AA^{-1}$, corresponds to a length of $l \approx 2.3 \AA$. This later value is however clearly smaller than the location of the first peak in $g_{N i N i}(r)$ (about $2.7 \AA$ ), which shows that the conversion relation above gives underestimated length values (by about 20\%). This can be further confirmed in the case of Ni-Zr and Zr-Zr correlations. Taking into account this correction, we obtain in the case of the prepeak a correlation length of about $3.9 \AA$. It seems plausible to attribute this length to the first split of the second peak in $g_{N i N i}(r)$. Furthermore the slight hump at the left end of the prepeak, which is independent of the truncation value used for the Fourier transform, correlates well with the second split. Note that there is no prepeak in the structure factor $S_{Z r Z r}^{F Z}(q)$.

The appearance of a prepeak in $S_{N i N i}^{F Z}(q)$ reflects the existence of chemical shortrange order (CSRO) in the atomic configuration of the binary as well as the ternary system, in other words the preference for $\mathrm{Ni}$ atoms to be surrounded by Zr atoms as discussed in the last section. The chemical nature of this SRO can be seen in the BT structure factors shown in Fig. (5.8). A peak at the same location appears only in the concentration-concentration structure factor $S_{c c}^{B T}(q)$ and not in the numberdensity structure factor $S_{N N}^{B T}$, nor in the cross term $S_{N c}^{B T}(q)$.

In the subsystem $\mathrm{Zr}-\mathrm{Al}$, the $\mathrm{FZ}$ structure factor $S_{A l A l}^{F Z}(q)$ also exhibits a well defined prepeak at a wave vector $q \approx 1.3 \AA^{-1}$, a clear signature of a structural order on a length scale of about $5.8 \AA$ (corrected value). The peak related to the Al-Al nearest-neighbor distance is around $q \approx 2.6 \AA^{-1}$, we note also the unusual width of this peak. The BT structure factor $S_{c c}^{B T}(q)$ in Fig. 5.8 exhibits correlations at the same wave vectors. Thus, the prepeak in this case describes a concentration fluctuation, as well.

In the concentration-concentration structure factor $S_{c c}^{B T}(q)$ of the Ni-Al subsystem, both prepeaks are seen as expected. Note that the little shoulder at the lefthand side is not an artefact of the Fourier transform, it persists to appear independently of the truncation value used (the maximum value is about $30 \AA) . S_{N N}^{B T}(q)$ has also a large asymmetric prepeak, corresponding to the $\mathrm{Ni}-\mathrm{Ni}$ correlation, with a shoulder which extends down to the position of the $\mathrm{Al}-\mathrm{Al}$ prepeak. It goes without saying that the relative potentials in the $\mathrm{Ni}-\mathrm{Al}$ subsytem play only a small role in the struc- 
tural order detected in this subsystem. More decisive is the relation (interaction) of $\mathrm{Ni}$ and $\mathrm{Al}$ atoms to the majority species $\mathrm{Zr}$.

Thus, both prepeaks which emerge from the q-space structural analysis are associated with concentration fluctuations in the amorphous ternary system. The question how to describe these fluctuations in terms of atomic arrangements can be answered in the light of the results of the two last sections. In the picture of the trigonal-prismatic structural ordering (of the subsystem Ni-Zr), the location of the prepeak in the $\mathrm{Ni}-\mathrm{Ni}$ correlation may be related to an average distance between neighboring trigonal prisms, where the $\mathrm{Ni}$ atoms occupy the centres of these prisms. The $\mathrm{Al}$ atoms are then constrained to group in the space between these Ni-Zr units, this can explain the structural inhomogeneity with the characteristic periodicity of about $5.8 \AA$ in the $\mathrm{Al}-\mathrm{Al}$ correlation. This is approximatly the distance between two $\mathrm{Al}$ groupings separated by a $\mathrm{Ni}-\mathrm{Zr}$ unit.

It should be, however, remarked that the absence of a prepeak in the FZ structure factor $S_{Z r Z r}^{F Z}(q)$ and the density-density structure factor $S_{N N}^{B T}$ seems to be in contrast with the suggested structural picture, which suppose clearly a certain topological structural ordering in the amorphous atomic pattern. This may be due to the fact that only a small fraction of the $\mathrm{Zr}$ atoms are involved in the trigonal prismatic local ordering because of the low Ni concentration. MD simulation [57] and experiments [64, 76] on binary $\mathrm{Ni}-\mathrm{Zr}$ alloys also show that the amorphous $\mathrm{Ni}_{33} \mathrm{Zr}_{67}$ does not exhibit a prepeak in $S_{Z r Z r}^{F Z}(q)$ and $S_{N N}^{B T}(q)$ structure factors, while amorphous $\mathrm{Ni}_{50} \mathrm{Zr}_{50}$ and $\mathrm{Ni}_{65} \mathrm{Zr}_{35}$ do. The fact that the two prepeaks appear in $S_{N N}^{B T}(q)$ and $S_{c c}^{B T}(q)$ of the subsystem $\mathrm{Ni}-\mathrm{Al}$, where the relative $\mathrm{Ni}$ and $\mathrm{Al}$ concentrations are comparable, provides support to this supposition.

\subsection{Temperature dependence of the prepeaks}

The temperature dependence of the prepeaks from the glassy state up to $T=5000 \mathrm{~K}$ is displayed on Fig. 5.10). The intensity of both prepeaks increases continously upon cooling, marking thereby the increase of the structural ordering with decreasing temperature. A noticeable aspect in this ordering effect is the unusual strong decrease of the first peak of $g_{N i N i}(r)$ in Fig. (5.9) upon cooling, the second peak getting thereby higher and more structured. The vanishing of the Ni nearest-neighbors manifests clearly the fact that, on cooling, the $\mathrm{Ni}$ atoms are confined at the center of their specific structural units, having ultimately only $\mathrm{Zr}$ or $\mathrm{Al}$ nearest-neighbors.

Another conspicuous feature which emerge from Fig. (5.10) is that the prepeaks are observed even at temperatures as high as $T=5000 \mathrm{~K}$, which indicates that the structural organization develops already at very high temperatures. This contrasts with the conventional conception that the atoms in the melt are randomly distributed. The persistence of a well-defined SRO even far obove the melting temperature seems to be a common feature of glass formers with bonding forces of 


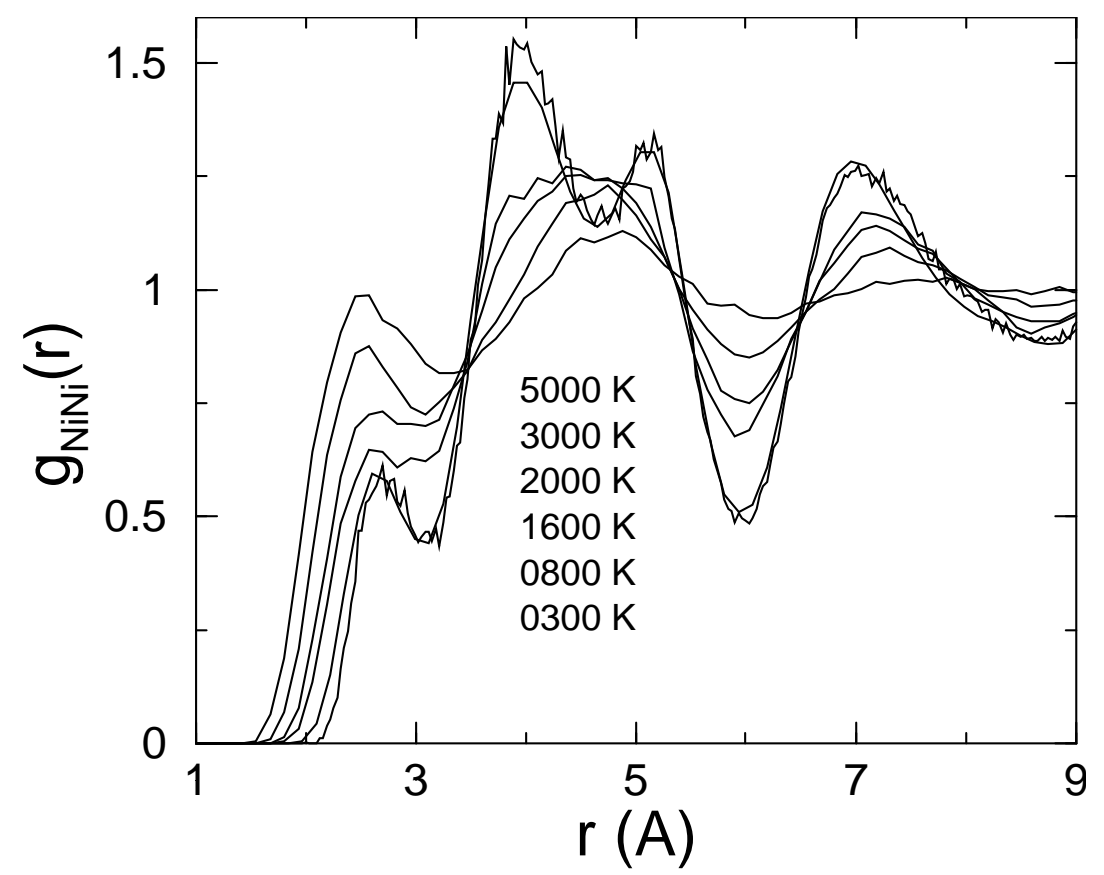

Figure 5.9: Temperature dependence of the PDF $g_{N i N i}(r)$. The indicated temperatures correspond to the sequence of the curves from the top to the bottom as given at the first peak around $2.7 \AA$.

covalent nature, like network-forming and transition-metals glassy systems [78]. 


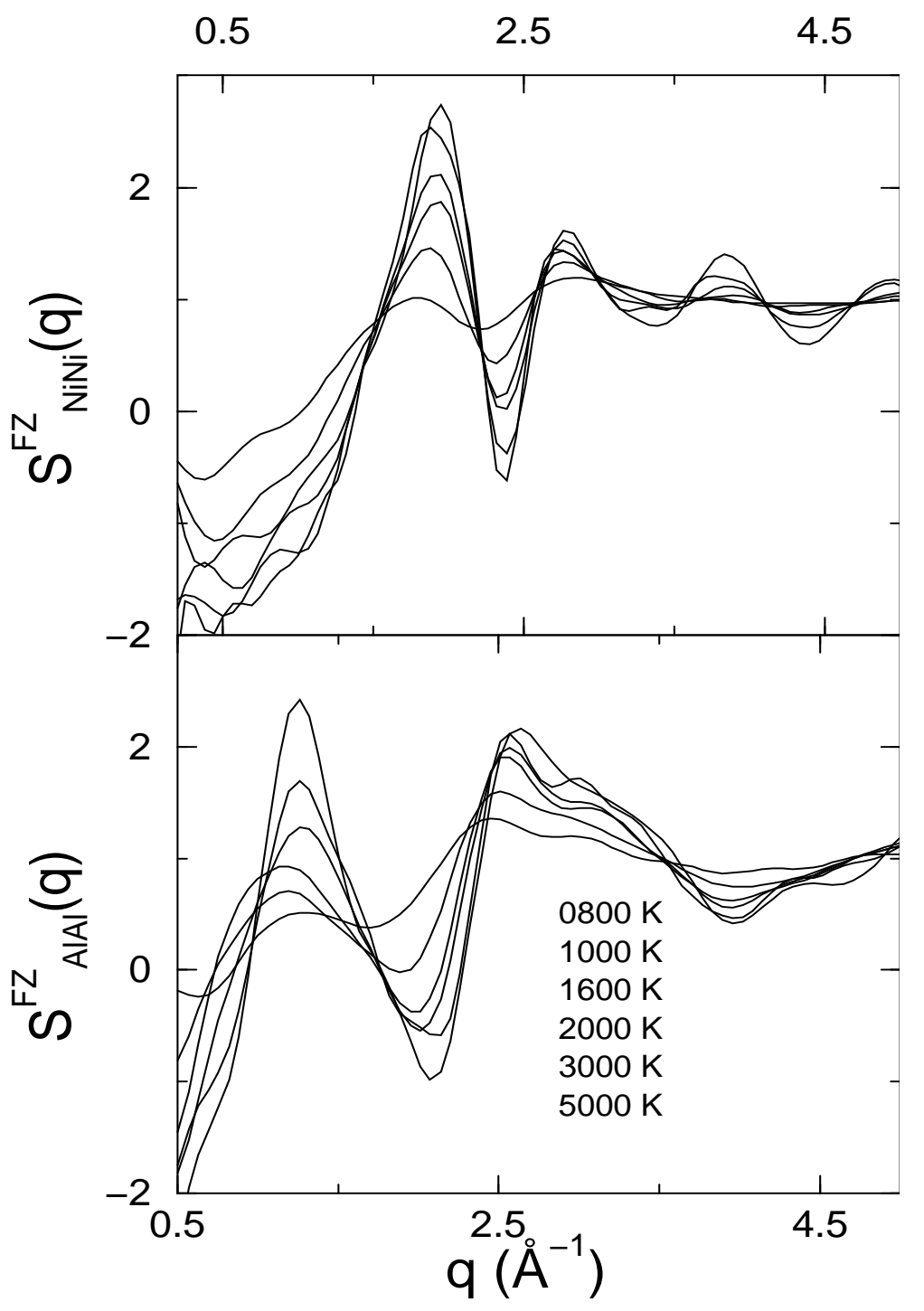

Figure 5.10: Temperature dependence of the prepeaks associated with the $\mathrm{Ni}-\mathrm{Ni}$ and $\mathrm{Al}-\mathrm{Al}$ correlations. The indicated temperatures correspond to the sequence of the curves from the top to the bottom as given at the prepeak (first peak) position. 


\section{Chapter 6}

\section{Dynamic properties}

Dynamic properties of the simulated $\mathrm{Ni}_{25} \mathrm{Zr}_{60} \mathrm{Al}_{15}$ system are presented in this chapter. The investigations concern the self-diffusion, the van Hove correlation functions, the intermediate scattering function, and the shear viscosity. A comparison with the binary $\mathrm{Ni}_{35} \mathrm{Zr}_{65}$ alloy is provided in the last section.

\subsection{Diffusion}

In MD simulations diffusion is studied by monitoring the mean square displacement (MSD) of a tagged particle from its initial position as a function of time. The diffusion constants $D_{\alpha}(\alpha=\mathrm{Ni}, \mathrm{Zr}, \mathrm{Al})$ are calculated from the long time behavior of the MSD where it exhibits a linear increase according to Einstein's law

$$
D_{\alpha}(T)=\frac{1}{6} \lim _{t \rightarrow \infty} \partial_{t}\left\langle\left(\mathbf{r}_{i}\left(t_{0}+t\right)-\mathbf{r}_{i}\left(t_{0}\right)\right)^{2}\right\rangle_{i, t_{0}} .
$$

The averaging is carried out over initial times $t_{0}$ and over all particles of the same species to get a good statistical accuracy.

In Fig. (6.1) we show the time dependence of the MSD for different temperatures in double logarithmic scale. Since the MSD presents similar features for the three species, we choose to show as illustration the results for one species (Ni) only.

At higher temperatures, i.e in the liquid state, the linear behavior is reached after a microscopic time, typically a few Einstein-vibration periods $(\approx 1 \mathrm{ps})$. Before colliding with their neighbors, the particles follow a free ballistic motion which explains the quadratic dependence on time of the MSD $\left(<r^{2}>\propto t^{2}\right)$ at very short times. Upon entering in the supercooled liquid region, i.e approaching the GT, a transient regime builds up between the ballistic and the diffusive motions. This manifests itself in a plateau at intermediate times, becoming larger with decreasing temperature and extending over several time decades at the lowest temperatures.

The plateau reflects that, during this time, each moving particle is temporary trapped in the cage formed by the surrounding neighbors. As the temperature is lowered, the cage becomes more and more rigid (due to increased viscosity) and only 


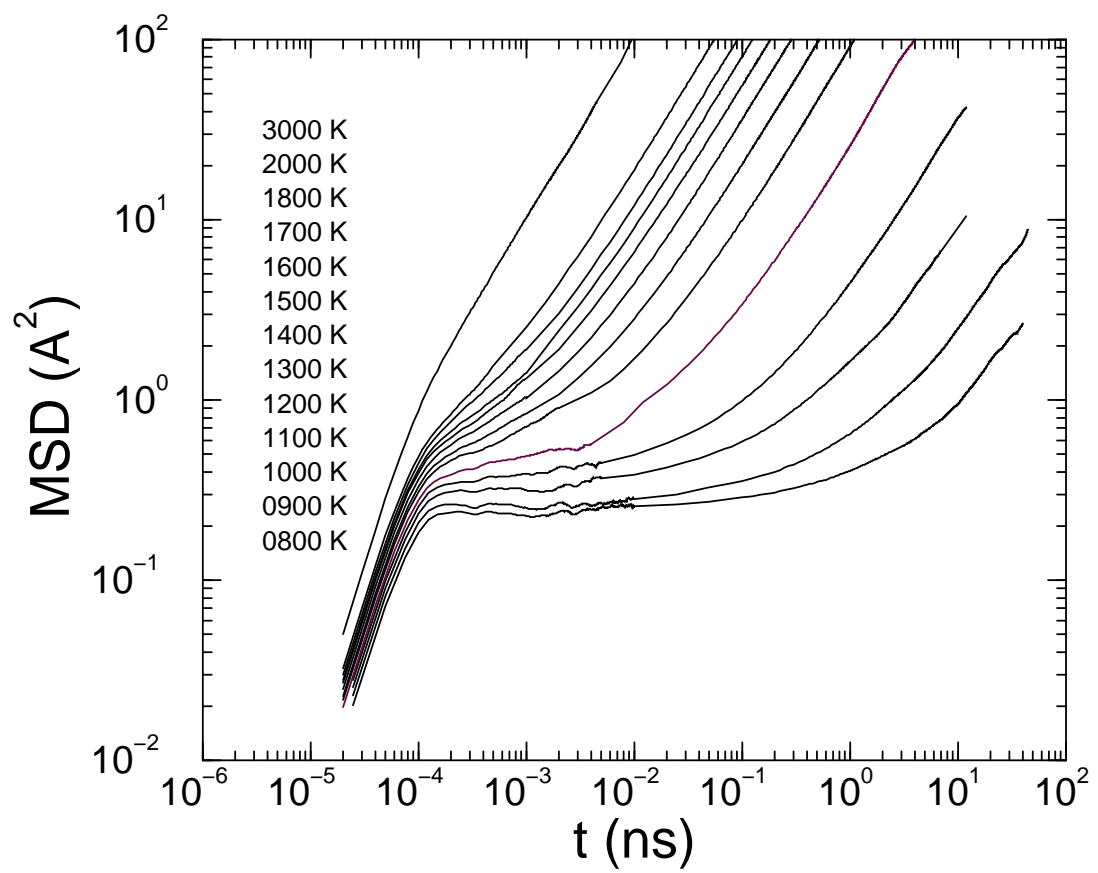

Figure 6.1: Time dependence of the mean squared displacement of $\mathrm{Ni}$ in $\mathrm{Ni}_{25} \mathrm{Zr}_{60} \mathrm{Al}_{15}$ for different temperatures. The ballistic time regime $<r^{2}>\propto t^{2}$ and the diffusive one $<r^{2}>\propto t$ are clearly resolved.

for larger times this cage starts to break up. Then the particles begin to diffuse and the MSD increases anew. The temperature $T=800 \mathrm{~K}$ is the lowest one where it was possible to reach the linear regime with our computer ressources. Below this temperature the particles remain practically confined in their cages and no diffusion could be resolved within the observation time.

In Fig. 6.2), we display the temperature dependence of the diffusion constants for the three species as gained from the MSD according to Eq. 6.1). This plot covers a very large temperature range up to $5000 \mathrm{~K}$ and 8 orders of magnitude of diffusivity. The first conspicuous observation from this plot is that the diffusion constants scale with the atom size upon the whole temperature range. At very high temperatures, we observe the relations $D_{N i} \approx D_{A l}$ and $D_{Z r} \approx 2 D_{N i}$. This scaling is reminiscent of the size and mass dependence of the diffusivity for a gas of hard spheres, whose size $\sigma$ is small in comparison to their mean free path, so that their movement is governed by uncorrelated binary collisions [82]

$$
D \propto \frac{1}{\sigma^{2} \sqrt{m}} .
$$

The fact that $D_{N i}$ and $D_{A l}$ are similar seems to be a compensation effect of the larger diameter $\sigma_{A l}$ but smaller mass $m_{A l}$. The ratio of $D_{N i}$ and $D_{Z r}$ at very high temperatures seems to be also in agreement with Eq. 6.2.

The three diffusion curves exhibit a change in the slope at the temperature $T \approx 1000 K$ which we identified in chapter 4 as the caloric glass temperature $T_{g}$. 


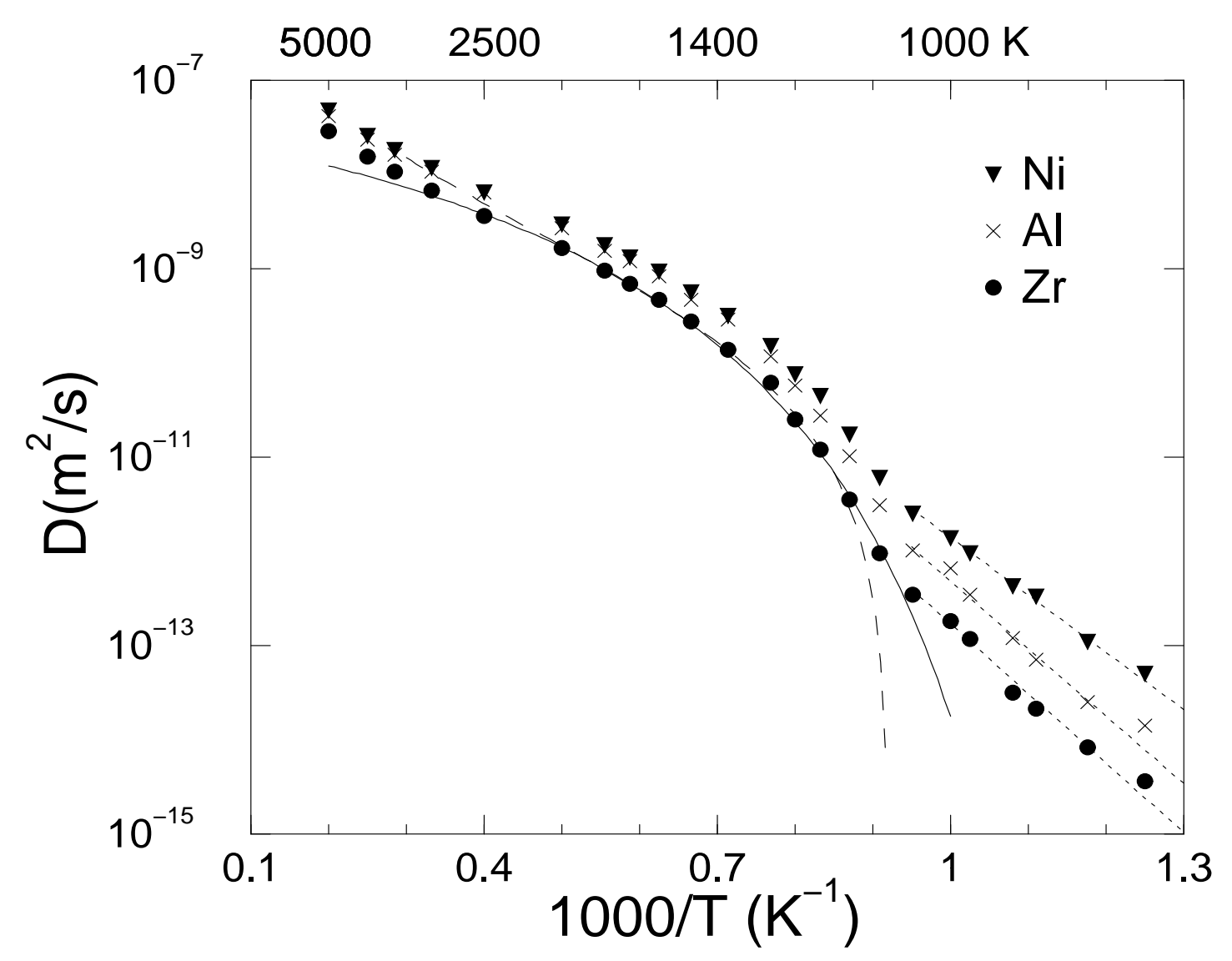

Figure 6.2: Arrhenius plot of the diffusion constants in $\mathrm{Ni}_{25} \mathrm{Zr}_{60} \mathrm{Al}_{15}$. The solid line represents a fit to the high-temperature data with the Vogel-Fulcher law. The dashed line is a fit with the power law predicted by the MCT. In order not to encomber the figure, we show these fits for the $\mathrm{Zr}$ data only. The dotted lines are Arrhenius fits to the data at low temperatures. 
Note, that while at higher temperatures the three diffusion constants run parallel to each other and show a very slow temperature dependence, in the highly undercooled region and specially upon approaching $T_{g}$, the ratios of the different $D_{\alpha} s$ grow up continuously and a faster decreasing of the diffusivity with temperature is enregistered for each species. Between $2500 K$ and $1400 K$ we observe a variation of approximately one decade against one decade between $1200 \mathrm{~K}$ and $1100 \mathrm{~K}$.

It is worth noticing that this drastic change upon crossing the GT region, refered to in the literature as the "slowing down", contrasts clearly with the smooth variation of the thermodynamic properties (e.g enthalpy and volume) and of the structural quantities (e.g. PDF $g_{i j}(r)$ ) in the same region as shown in the previous chapters. This ambivalent behaviour seems to be a fundamental feature common to all amorphous materials [83].

\subsubsection{Self-diffusion in the liquid state}

We consider now the temperature dependence of the diffusion constants. At a first sight, we can say that $T_{g}$ separates the temperature scale in two regions where the $D_{\alpha} s$ exhibit different T-dependences: a curved behaviour above $T_{g}$ and a linear one below, when represented in an Arrhenius plot. Above $T_{g}$ we have used, on the one hand, a power law fitting

$$
D \propto\left|T-T_{c}\right|^{\gamma}
$$

in order to test this central prediction of the MCT [24]. On the other hand, we have fitted the data with the Vogel-Fulcher law

$$
D \propto \exp \left(-B /\left(T-T_{0}\right)\right)
$$

which is known phenomenologically to describe quite well the temperature dependance of some transport coefficients in the supercooled region of melts.

As shown by the dashed line in Fig. (6.2), the power law seems to fit very well the diffusion data over a relatively wide temperature range of about $1000 \mathrm{~K}$ and over about 3 orders of magnitude in $D_{\alpha}$. The critical temperature $T_{c}$ and the exponent $\gamma$, listed in tab.6.1), are apparently independent (within a precision of $2 \%$ ) of the particle species, which is in agreement with the predictions of the MCT. The slight scaling of $T_{c}$ with the species size is not fortuitous but may be an effect resulting from the existence of hopping processes which affect differently the three species. We will come back to the subject of the hopping processes in more details in the next sections.

It is known that by using extremely high cooling rates in MD simulations, the calorimetric GT temperature $T_{g}$ may be raised to such an extent that it lies above $T_{c}$, eclipsing thereby the kinetik GT [84]. We find hier a $T_{c}$ which is slightly above 
$T_{g}$. This indicates that the cooling rate we applied in this work is sufficiently low to allow a meaningful study of the kinetik GT in the sense of the MCT.

Table 6.1: Parameters of the power law (after MCT), Vogel-Fulcher and Arrhenius fits for the diffusion constants. The power law and VF relation fit the diffusivity data above $T_{g}$, while the Arrhenius fit is for data below $T_{g}$.

\begin{tabular}{ccccccc}
\hline \hline & \multicolumn{2}{c}{ MCT } & \multicolumn{2}{c}{ VF } & \multicolumn{2}{c}{ Arrhenius } \\
\hline & $T_{c}[K]$ & $\gamma$ & $B[K]$ & $T_{0}[K]$ & $D_{0}\left[10^{-6} m^{2} / s\right]$ & $\mathrm{Q}[\mathrm{eV}]$ \\
\hline $\mathrm{Ni}$ & 1045 & 2.45 & 3500 & 700 & 1.7 & 1.12 \\
$\mathrm{Zr}$ & 1085 & 2.41 & 3500 & 755 & 6.5 & 1.36 \\
$\mathrm{Al}$ & 1062 & 2.40 & 3490 & 730 & 21 & 1.31 \\
\hline \hline
\end{tabular}

The VF law gives a good description of the diffusivity, covering even one decade more of the $D_{\alpha} s$ and a larger temperature range than the power law. The fitting parameters are given in tab. 6.1). The "critical" temperature $T_{0}$ seems to scale with the mobility of the diffusing atom species. However, if $T_{0}$ could be identified with the so-called Kauzmann temperature [85, 86], then it must be the same for all atom species in the system.

For comparison, we note that the values of $T_{c}$ and $T_{0}$ we obtain here are very close to $T_{c}$ and the Kauzmann temperature $T_{K}$ obtained by Teichler [12, 15] from MD simulations of $\mathrm{Ni}_{50} \mathrm{Zr}_{50}$ based on Hausleitner-Hafner potentials: $T_{c}=1120 \mathrm{~K}$, $T_{K}=750 \mathrm{~K} . T_{c}$ was deduced from analyzing the self-part of the intermediate scattering function. $T_{K}$ was determined from thermodynamic considerations as the isentropic Kauzmann temperature.

\subsubsection{Self-diffusion in the glassy state}

We consider now the temperature dependence of the diffusivity below the glass temperature. As we know, $T_{g}$ marks the temperature region where the system falls out of equilibrium, i.e the relaxation time of the system around and below $T_{g}$ exceeds the time scale of the simulations, so that it is no longer possible to equilibrate the system toward a metastable state. On the scale of the simulation, the system may be perceived as a disordered nearly frozen structure, 'nearly' because residual diffusion events and aging still take place.

We achieved a fit to the $D_{\alpha} s$ below $T_{g}$ by an Arrhenius law

$$
D=D_{0} \exp \left(-Q / k_{B} T\right)
$$


which gives a good description of the data in this temperature range. The activation energy $\mathrm{Q}$ and the prefactor $D_{0}$ for each species are given in tab.6.1).

A common dynamic feature exhibited by nearly all amorphous alloys, is an Arrhenius law with constant activation energy $Q$ for the diffusivity in the relaxed glassy state [21]. This gives raise to the assumption that the diffusion in glasses is more related to the thermally activated diffusion in crystalline solids than to atomic dynamics in liquids. Our simulation is apparently able to reproduce this Arrhenius behavior in the temperature range of what we have called the glassy state on the time scale of the simulation. This fact is not obvious when we remember that there are approximately ten orders of magnitude between the time scale of a real experiment (minutes) and that of a simulation (10-100 ns). The Arrhenius behavior below $T_{g}$ has been reproduced as well in MD simulations by Teichler [30] for $\mathrm{Ni}_{50} \mathrm{Zr}_{50}$. He obtained an activation energy for Ni comparable to our $\left(Q_{N i} \approx 1.2 \mathrm{eV}\right)$. The experimental value [87] lies in the same range $Q_{N i}^{e x p} \approx 1.3 \mathrm{eV}$. The value of $Q_{Z r}$ obtained by Teichler lies however something higher $(\approx 2.0 \mathrm{~K})$ than the value from our model. Corresponding experimental results for Zr diffusion are not yet available.

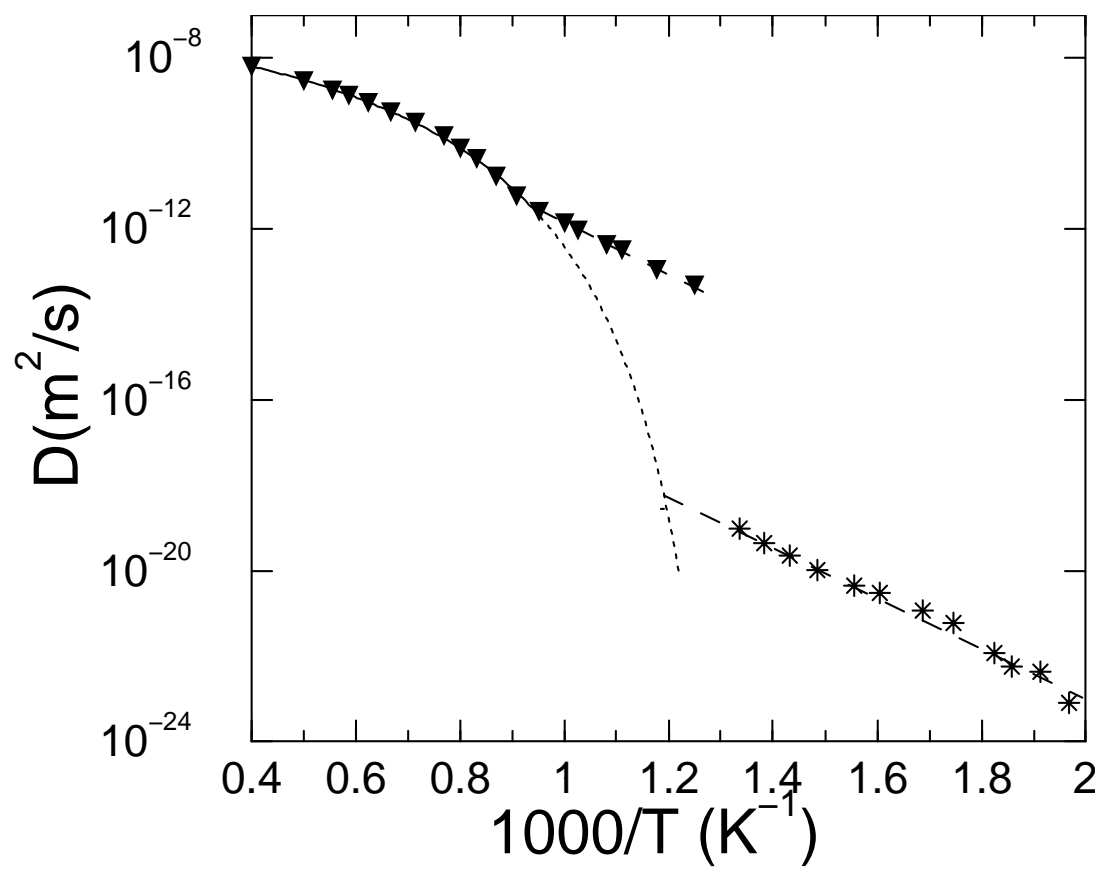

Figure 6.3: Arrhenius plot of the diffusion constant of $\mathrm{Ni}$ in $\mathrm{Ni}_{25} \mathrm{Zr}_{60} \mathrm{Al}_{15}$ as calculated from our MD simulation (triangles) and the experimental values for Co tracer diffusion in amorphous $\mathrm{Ni}_{23} \mathrm{Zr}_{62} \mathrm{Al}_{15}$ (asterisks) as measured by Hahn et al. [88. The dotted line is an extrapolation of our data for the melt to lower temperatures with the Vogel-Fulcher law. 


\subsubsection{Comparison with experimental results}

In Chapter 5 we have seen that our model gives a realistic description of the structural properties of amorphous $\mathrm{Ni}_{25} \mathrm{Zr}_{60} \mathrm{Al}_{15}$. It would be very instructive to know whether this model is able to describe also the dynamical properties, like diffusion, in a reasonable way. Unfortunately, no experimental data about the temperature dependence of self-diffusion in this system or in a comparable concentration are known to us. Very recently, Hahn and Flege [88] carried out various tracer diffusion measurements in amorphous NiZrAl alloys, among them Cobalt tracer diffusion in amorphous $\mathrm{Ni}_{23} \mathrm{Zr}_{62} \mathrm{Al}_{15}$. Due to the chemical and size similarity of $\mathrm{Ni}$ and $\mathrm{Co}$ atoms, it is expected that $\mathrm{Ni}$ and Co have similar mobilities. Moreover, in most known cases, these two atom species show diffusion coefficients close to each other. For this reason we compare in Fig. (6.3) our calculated $D_{N i}$ with $D_{C o}$ measured by Flege and Hahn. The large gap between the temperature range of our calculations and that of the experimental results makes difficult a direct comparison of the two data sets. The extrapolation of our results below $T_{g}$ to lower temperatures leads to diffusivity values which are four decades larger than the experimental ones. This extrapolation, however, has to be considered with caution because we compare two data sets corresponding to two different cooling rates. Our inevitably large cooling rate may be the reason for the discrepancy.

Extrapolation of our high temperature equilibrium data to lower temperatures along the Vogel-Fulcher curve, considered as independent on the cooling rate, and construction of its intersection with the Arrhenius extrapolation of the results of Hahn and Flege gives an estimation of the GT temperature $T_{g}$ as measured at cooling rates typical for laboratories. With this construction we obtain a $T_{g}$ of 840 $\mathrm{K}$, which overestimates the experimental GT temperature by $15 \%\left(T_{g, \exp }=720 \mathrm{~K}\right.$ 4]) only.

\subsection{Van Hove Correlation function}

\subsubsection{Definition 89}

The correlation between the densities at two points separated by $\mathbf{r}$ and $\mathrm{t}$ in space and time, respectively, in a fluid is defined as

$$
G\left(\mathbf{r}, \mathbf{r}^{\prime}, t\right)=\frac{1}{N}<\rho\left(\mathbf{r}+\mathbf{r}^{\prime}, t\right) \rho\left(\mathbf{r}^{\prime}, 0\right)>,
$$

where $\mathbf{r}^{\prime}$ is an arbitrary origin point, and $\rho(\mathbf{r}, t)$ the number density, at point $\mathbf{r}$ and time $t$, which can be defined microscopically as

$$
\rho(\mathbf{r}, t)=\sum_{i=1}^{N} \delta\left[\mathbf{r}-\mathbf{r}_{i}^{\prime}(t)\right]
$$


For homogeneous fluids, a non-local correlation function depends only on the relative coordinates and not on the choice of the origin. This allows in our case to average over $\mathbf{r}^{\prime}$ giving

$$
\begin{aligned}
G(\mathbf{r}, t) & =\frac{1}{N}\left\langle\sum_{i=1}^{N} \sum_{j=1}^{N} \int \delta\left[\mathbf{r}^{\prime}+\mathbf{r}-\mathbf{r}_{i}(t)\right] \delta\left[\mathbf{r}^{\prime}-\mathbf{r}_{j}(0)\right]\right\rangle \\
& =\frac{1}{N}\left\langle\sum_{i=1}^{N} \sum_{j=1}^{N} \delta\left[\mathbf{r}+\mathbf{r}_{j}(0)-\mathbf{r}_{i}(t)\right]\right\rangle
\end{aligned}
$$

The function $G(\mathbf{r}, t)$, introduced first by van Hove, separates naturally into two terms, usually called the "self" $(i=j)$ and "distinct" $(i \neq j)$ parts

$$
G(\mathbf{r}, t)=G^{s}(\mathbf{r}, t)+G^{d}(\mathbf{r}, t) .
$$

$G(\mathbf{r}, t) d \mathbf{r}$ gives the probability to find a particle $i$ in a volume element $d \mathbf{r}$ around the point $\mathbf{r}$ at time $t$ given that there was a particle $j$ at the origin at time $t=0$; the division into self and distinct parts corresponds to the two cases where $i$ and $j$ are the same particles or different ones, respectively. $G^{s}$ probes the dynamic of a single particle in terms of its displacement from an initial position whereas $G^{d}$ describes the collective character of the dynamics. For isotropic fluids, $G^{s}$ and $G^{d}$ depend only on the scalar quantity $r$.

In the long-wavelength, low-frequency limit (hydrodynamic limit) the Van Hove self correlation function writes

$$
G^{s}(r, t)=\frac{1}{(4 \pi D t)^{3 / 2}} \exp \left(-\frac{r^{2}}{4 D t}\right)
$$

where $D$ is the self-diffusion constant.

Rather than considering the density-density correlation in real space, it is often more convenient to deal with its representation in $\mathbf{k}$-space $F(\mathbf{k}, t)$, which is called the intermediate scattering function. The spectrum of $F(\mathbf{k}, t)$, defined as

$$
S(\mathbf{k}, \omega)=\frac{1}{2 \pi} \int_{-\infty}^{+\infty} F(\mathbf{k}, t) \exp (i \omega t)
$$

is the dynamic structure factor, a central quantity in inelastic neutron-scattering measurements.

\subsubsection{Van Hove self correlation function}

Fig. 6.4 shows the function $4 \pi r^{2} G_{\alpha}^{s}(r, t)$ for different times t at temperatures $T=1800 K$ and $T=1300 K$. At $T=1800 K$, which lies well above $T_{c}$, we see that the three species exhibit a hydrodynamic behavior, i.e. they show a single 

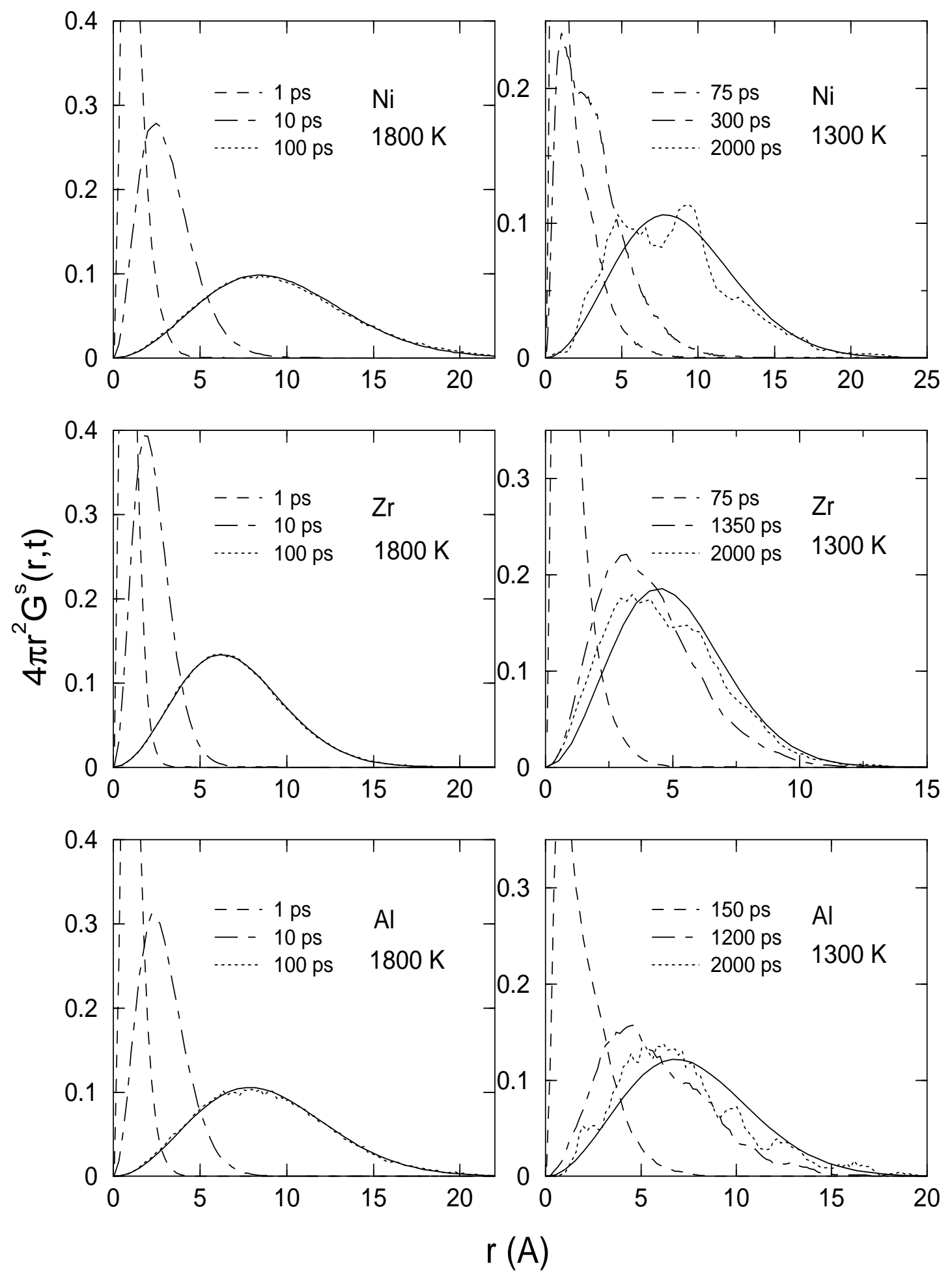

Figure 6.4: Space and time dependence of the self-part of the van Hove correlation function $4 \pi r^{2} G_{s}^{\alpha}(r, t)$ at $T=1800 \mathrm{~K}$ and $T=1300 \mathrm{~K}$. The solid lines represent the hydrodynamic limit (Eq. (6.10). 


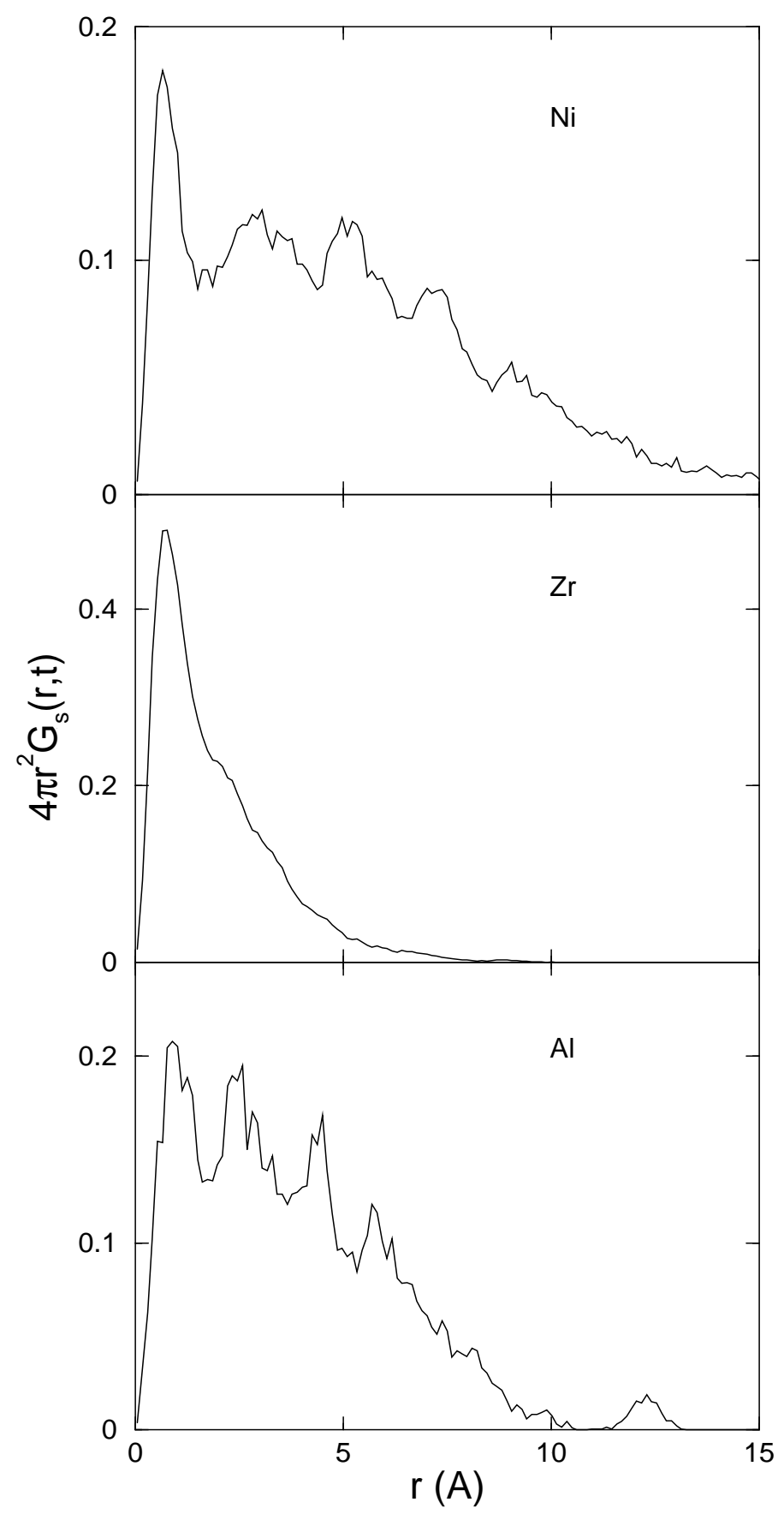

Figure 6.5: Space dependence of the self-part of the van Hove correlation function $4 \pi r^{2} G_{s}^{\alpha}(r, t)$ at $T=1100 K$ and $t=10 \mathrm{~ns}$. 


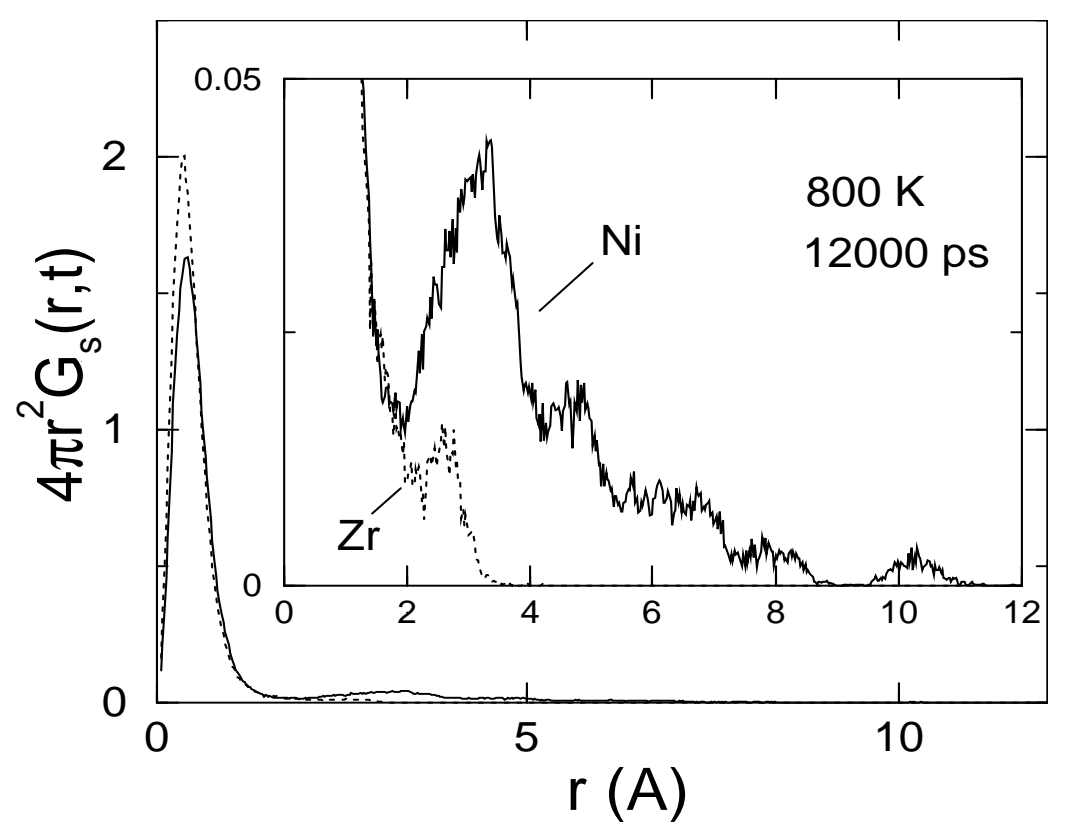

Figure 6.6: self-part of the van Hove correlation function $4 \pi r^{2} G_{s}^{\alpha}(r, t)$ at $T=$ $800 \mathrm{~K}$ after $t=12 \mathrm{~ns}$ for $\mathrm{Ni}$ (solid line) and $\mathrm{Zr}$ (dotted line). The inset is a 50 times enlarged picture of the curves at large distances.

gaussian-like peak broadening and shifting toward larger distances with increasing time. Thus the material transport proceeds at sufficiently higher temperatures via a continuous flow-like process as it is known for normal liquids. We have observed, moreover, that the hydrodynamic limit (Eq. (6.10) ) is effectively reached within a time comparable with the relaxation time of the system, i.e. the time after which density or shear stress fluctuations vanish (see sections 6.4 and 6.5).

At $T=1300 K$, the van Hove functions still exhibit a gaussian-like peak which broadens and moves to larger distances. The hydrodynamic limit is far from being reached even after a relatively long observation time of $2 n s$, which is larger than the relaxation time of the system at this temperature. More remaquable is the appearance of secondary peaks, especially in the case of $\mathrm{Ni}$ and $\mathrm{Al}$ atoms. Such a feature, reported first by Roux et al. [28] in their MD simulation of soft spheres, has been observed in many computer experiments [29, 30, and is conventionally attributed to the presence of thermally activated hopping processes.

As the temperature is further lowered, the hopping processes become more and more important as exhibited in Fig. 6.5 for $T=1100 K$, i.e. in close vicinity of $T_{c}$. The emergence of several distinct peaks at distances corresponding to multiples of the interparticle spacing now marks clearly the shape of $G_{\alpha}^{s}(r, t)$. This feature is much more pronounced for $\mathrm{Ni}$ and $\mathrm{Al}$ than for the larger $\mathrm{Zr}$ atoms. The immobility of the main peak at a fixed location reflects the presence of vibrating atoms trapped in their cages for long times, as discussed in the previous section. The intensity 
decrease of this peak with time is related to the rate of atoms which succeed to break their cages.

Fig. (6.6), taken at a temperature well below $T_{c}(T=900 \mathrm{~K})$, illustrates clearly how the diffusion in the glassy state is carried by a minority of mobile particles embedded in a rather frozen matrix. This is concluded from the extremely small contribution that develops at the foot of the vibrational peak but extends to distances of several nearest-neighbor distances. This coexistence of fast and slow mobility indicates the heterogeneous character of the diffusion in this temperature range. We note here that evidence for structural and dynamical heterogeneities in undercooled liquids and glasses recently has been found in MD simulations and experiments. We will discuss this subject again in section 7.3 below.

\subsubsection{Van Hove distinct correlation function}

We have seen in the previous chapter that the strucure of amorphous $\mathrm{Ni}_{25} \mathrm{Zr}_{60} \mathrm{Al}_{15}$ is far from being random and that a chemical and topological ordering is manifestely present even at high temperatures. Intuitively, we expect therefore that these well defined structural correlations reflect themselves in the dynamics as well, in form of correlated motions of the different particles. This means that if, e.g., a Ni atom with a trigonal prismatic local order makes a jump leaving a hole behind, then it is expected that this hole will be filled at late times by another Ni atom from the vicinity to preserve the established order. Motivated by this idea, we have calculated the distinct part of the Van Hove correlation function $G_{d}(r, t)$ between different particles of the same species. The above mentioned correlation feature is clearly demonstrated in the $G^{d}(r, t) s$ of Ni-Ni and Al-Al pairs at $T=1100 K$ (Fig. (6.7)), where for $t>0$ a strong peak has developed and grown with time at the origin $(r=0)$. This shows that the position of a moving original particle is occupied with high probability by another $\mathrm{Ni}$ or $\mathrm{Al}$, respectively, at later times. The correlation seems to be more pronounced for $\mathrm{Al}$ than for $\mathrm{Ni}$. This can be explained by the fact that a $\mathrm{Ni}$ atom is principally surrounded by the two others species $\mathrm{Zr}$ or Al, so that the opportunity to hop to a site previously occupied by a $\mathrm{Ni}$ atom will be restricted. Whereas for $\mathrm{Al}$ atoms the situation is different because of their tendency to form groups. Surprisingly, such a correlated motion is almost inexistent for Zr atoms, where the $G^{d}(r, t)$ displays a behavior similar to that of normal liquids at sufficient high temperatures. This observation provides support to a conclusion drawn already from the analysis of $G^{s}(r, t)$ in the last section, namely that the hopping processes are not the dominant mechanism in the diffusion of $\mathrm{Zr}$ atoms. Even in the region of the kinetik GT or below, the Zr matrix moves essentially via continuous viscous motion.

We see that the peak at the origin is observable at $t=8 \mathrm{~ns}$ (and even beyond), that means at times where the particles show a diffusion behavior and density or 


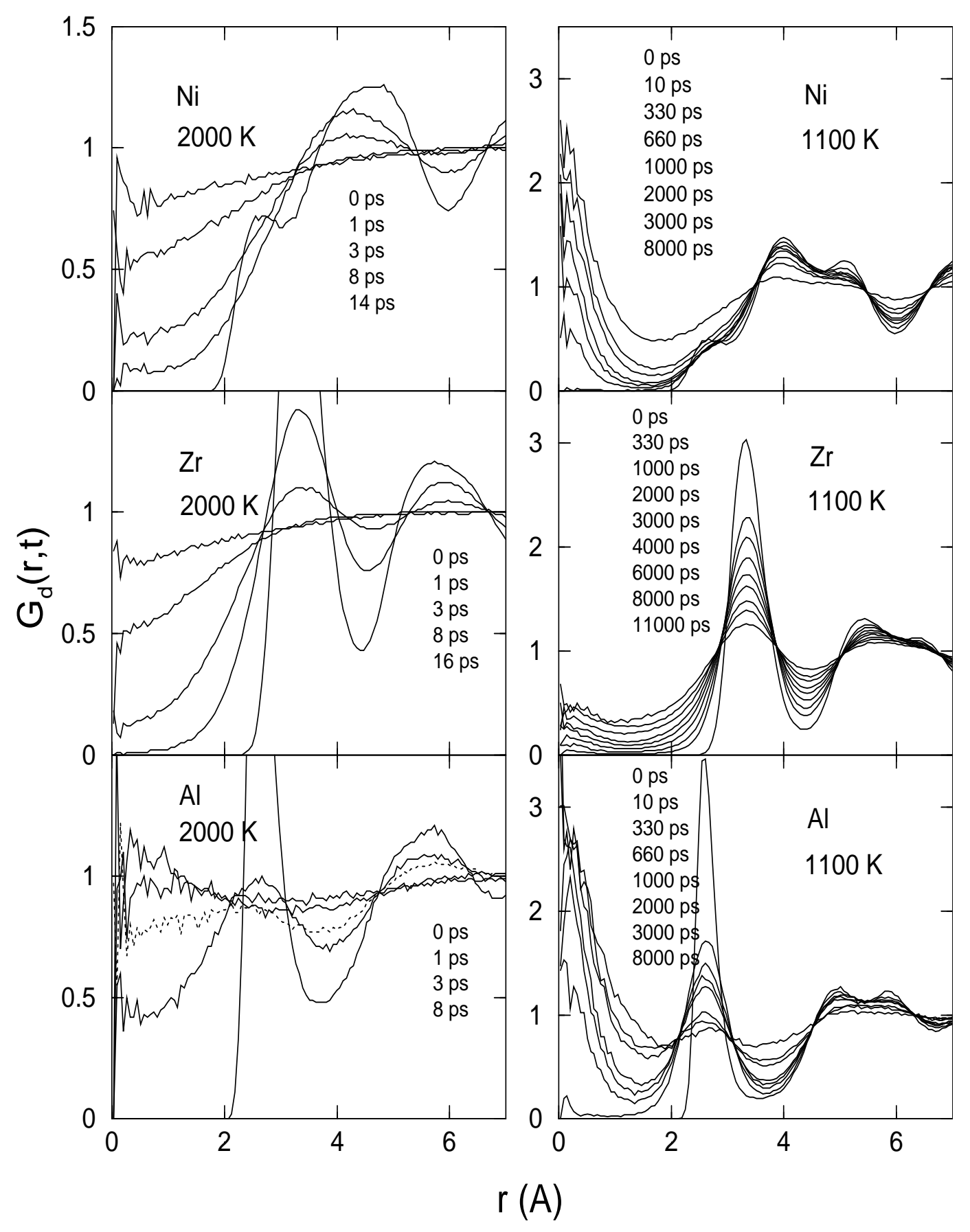

Figure 6.7: Space and time dependence of the distinct part of the van Hove correlation function $G_{\alpha}^{d}(r, t)$ at $T=2000 \mathrm{~K}$ and $T=1100 \mathrm{~K}$. 
shear stress fluctuations have almost vanished. This demonstrates that even after these relatively long times, the sites structure correlates strongly with the one at $t=0$. Another remarkable feature is the appearance, although weak, of the correlation peaks at $r=0$ even at the relatively high temperature of $T=2000 \mathrm{~K}$. This observation is in agreement with the existence of the prepeaks in the same temperature range, confirming once again that the structural organization develops already in the liquid state.

\subsection{Hopping and flow motion}

The analysis of the Van Hove correlation function has shown that hopping processes begin to take place actually at temperatures well above the kinetic GT, $T_{c}$. Upon crossing $T_{c}$, the hopping diffusion becomes more and more important but the viscous flow is still omnipresent even in the glassy state below $T_{g}$. This can be inferred from a slight but continuous broadening of the vibrational peak and a continuous shift with time of the hopping peaks to larger distances, which shows that the whole structure undergoes flow motion. In this section, we attempt to quantify the relative contribution of both diffusion modes, hopping and flow, in the total material transport and to establish how it varies upon crossing $T_{c}$.

We have to define first what we shall call a hopping event. For this we need a distance $\delta l$ and a specific time $\delta t$ within which the distance is crossed. We choose $\delta l$ as the location of the first minimum of $G^{s}(r, t)$ at sufficiently low temperatures $(T=800 \mathrm{~K})$ (Fig. (6.6)) to minimize as much as possible the contribution of the flow motion, this corresponds to $\delta l \approx 2.1 \AA$. A hopping is a thermal activated event which occurs on the time scale of the atomic vibration, we choose therefore $\delta t$ in the range of an Einstein vibration period $\delta l \approx 1 \mathrm{ps}$. This value is in agreement with the finding of Sieber [90] who showed, in a MD study on $\mathrm{Co}_{67} \mathrm{Zr}_{33}$, that in thermal activated hopping the saddle point was crossed within about 1 ps. Obviously, the distinction between flow and hopping diffusion makes sense only if the time scales of the last process is negligible compared to that of the former. This is indeed the case in the temperature range $(T<1300 K)$, where this analysis is carried out. At $T=1300 \mathrm{~K}$, for instance, where the diffusion seems to be dominated by a homogeneous viscous flow as displayed by the Van Hove correlation function in Fig. (6.4), the average time needed by a particle to cross the distance $\delta l$ is about $100 \mathrm{ps}$, i.e. two orders of magnitude larger than the time scale of a thermal activated jump.

To separate flow from hopping diffusion during an observation time $t$, we divide $t$ into elementary intervals of length $\delta t$ and the corresponding atomic displacements $\delta r_{i}$ are classified as hopping or flow events depending if they fulfill $\delta r_{i} \geq \delta l$ or $\delta r_{i} \leq \delta l$, respectively. The elementary displacements are then summed up to build the MSDs, from which the diffusion constants $D_{h}$ and $D_{f}$ for the hopping and flow motions, respectively, are evaluated according to Eq. (6.1). 


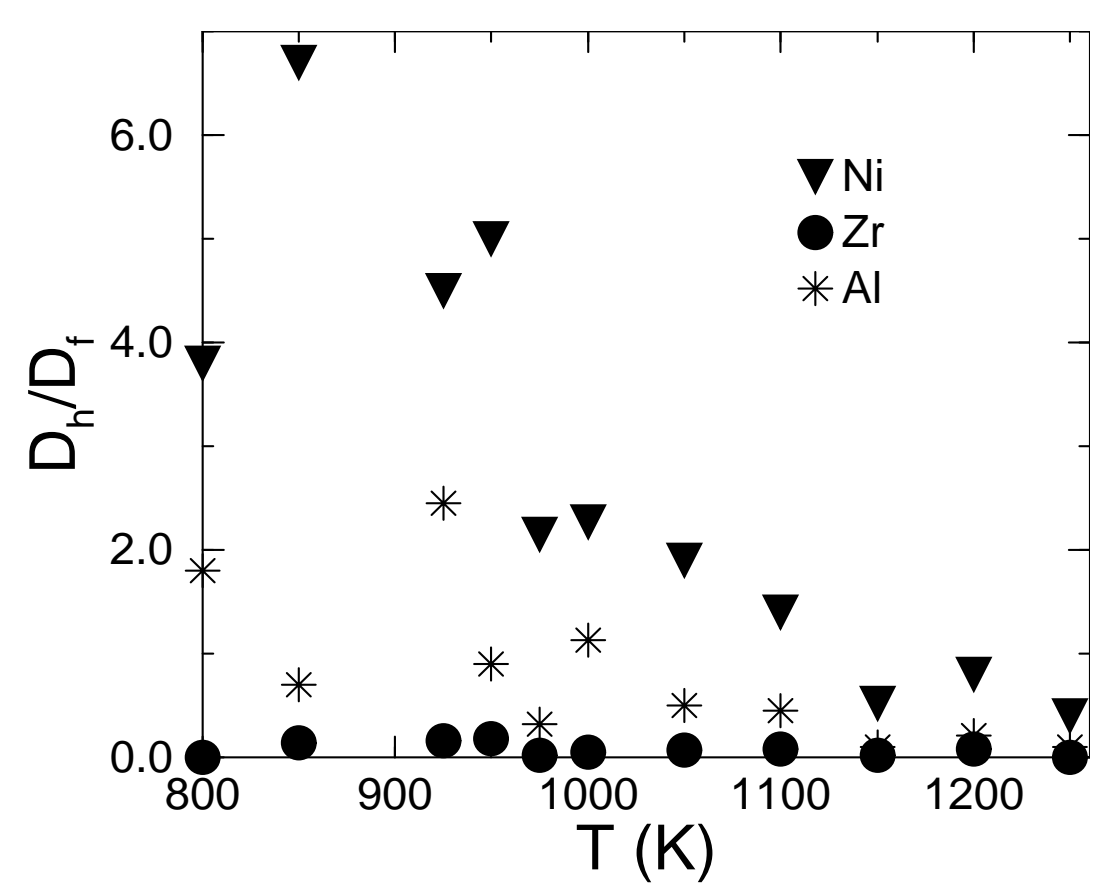

Figure 6.8: Temperature dependence of the ratio $D_{h} / D_{f}$, where $D_{h}\left(D_{f}\right.$

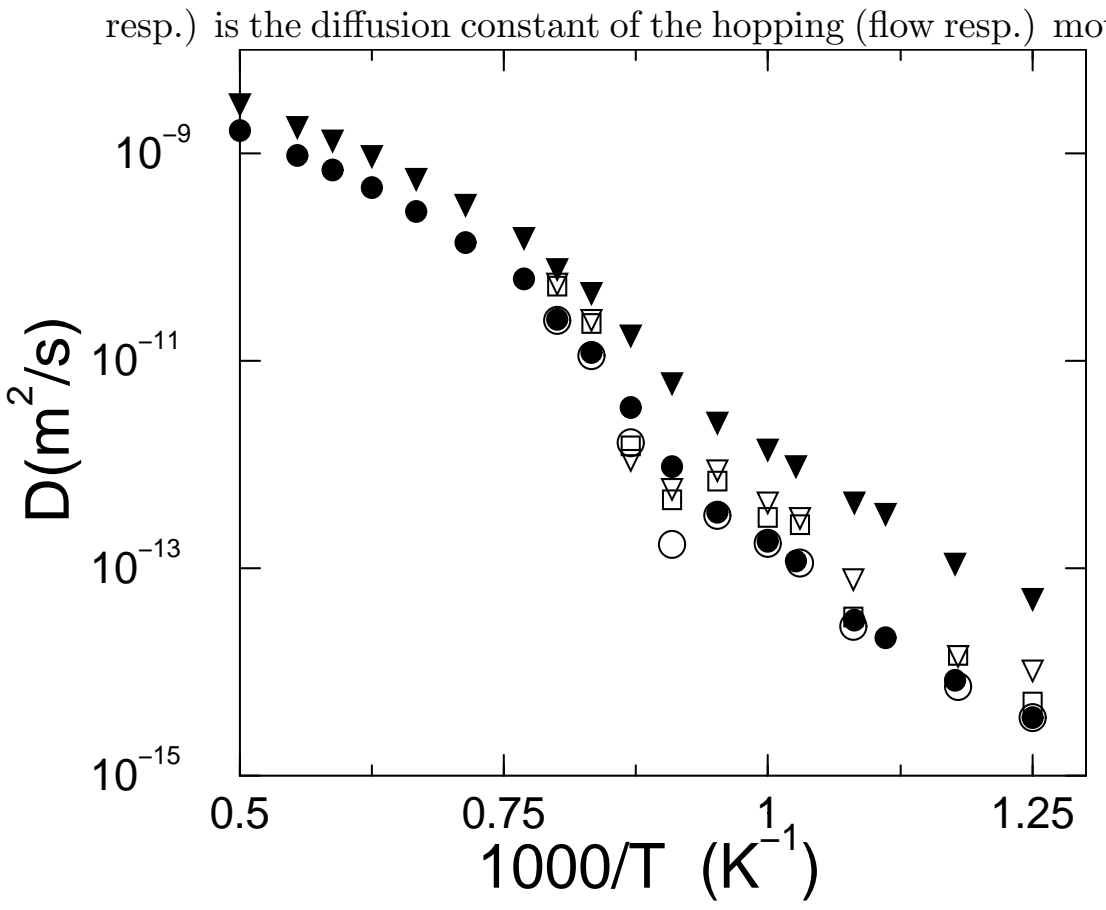

Figure 6.9: Arrhenius plot of the diffusion constants in $\mathrm{Ni}_{25} \mathrm{Zr}_{60} \mathrm{Al}_{15}$. The full symbols represent the total diffusion constants as given in Fig. (6.2), the open ones are the diffusion constants of the flow motion. 
We display in fig(6.8) the temperature dependence of the ratio $D_{h} / D_{f}$ from lower temperatures up to the temperature range $(T=1300 K)$, where a distinction between a flow and a hopping event after our convention becomes impossible. We see that the behavior of the three species is manifestly different. While for Ni with decreasing temperature the hopping diffusion gets more and more effective compared to flow diffusion, the flow remains for Zr atoms the main diffusion mode over the whole temperature range investigated. A slight increase of the ratio $D_{h} / D_{f}$ can be observed for $\mathrm{Zr}$ down to $T_{c}$, neverthless below $T_{c}$ only flow can be detected, in essence. Al atoms exhibit a behavior similar to that of $\mathrm{Ni}$ albeit less pronounced. An extrapolation to further lower temperatures seems to be obvious for $\mathrm{Ni}$ and $\mathrm{Al}$, where the curves suggest a continuous increase of $D_{h}$ relatively to $D_{f}$. For Zr atoms, the behavior of the curve below $T_{c}$ suggests that the $\mathrm{Zr}$ diffusion is essentially carried by a flow motion even at lower temperatures.

In Fig. 6.9 we display the Arrhenius plot of the flow diffusion constants $D_{h}$ for the three species and additionally the total diffusion coefficients $D_{N i}$ and $D_{Z r}$, while $D_{A l}$ has been omitted to not overburden the figure. We see that considering only the flow diffusivity, the parallel run of the three total diffusion constants at higher temperatures is restored for the lower temperatures. This suggests that the flow-like motion, at lower temperatures, proceeds in the same manner as the viscous flow in the liquid state at higher temperatures, i.e. a collective motion where many particles are involved, independently of their nature. The activation energy for this diffusion mode is thus the same for the three species. Gaukel et al. [91, 92] arrived at a similar conclusion in a MD simulation of $\mathrm{Cu}_{33} \mathrm{Zr}_{67}$ system modelled with EAM interatomic potentials.

\subsection{Intermediate scattering function}

Structural relaxations and the slowing down of the dynamics near $T_{c}$ may be conveniently characterized by analysing the time dependence of the intermediate scattering function $F(q, t)$ [89] for wave lengths near the interparticle spacing, i.e. for wave numbers $q$ near the main peak of the static structure factor. In this range of wave numbers, $F(q, t)$ and its self-part $F_{s}(q, t)$ exhibit a very similar behaviour, so that we will restrict ourselves here to examine $F_{s}(q, t)$, which can be, moreover, calculated with a higher statistical accuracy due to the additional averaging over all particles.

We compute $F_{s}(q, t)$ from the positions of the particles [89]

$$
F_{s}(\mathbf{q}, t)=\left\langle e^{i \mathbf{q} \cdot\left[\mathbf{r}_{i}\left(t_{0}+t\right)-\mathbf{r}_{i}\left(t_{0}\right)\right]}\right\rangle_{i, t_{0}}
$$

$F_{s}(q, t)$ might be, alternatively, calculated by taking the Fourier transform of the Van Hove correlation function $G^{s}(r, t)$ [89]. This method has, however, the disadvantage 


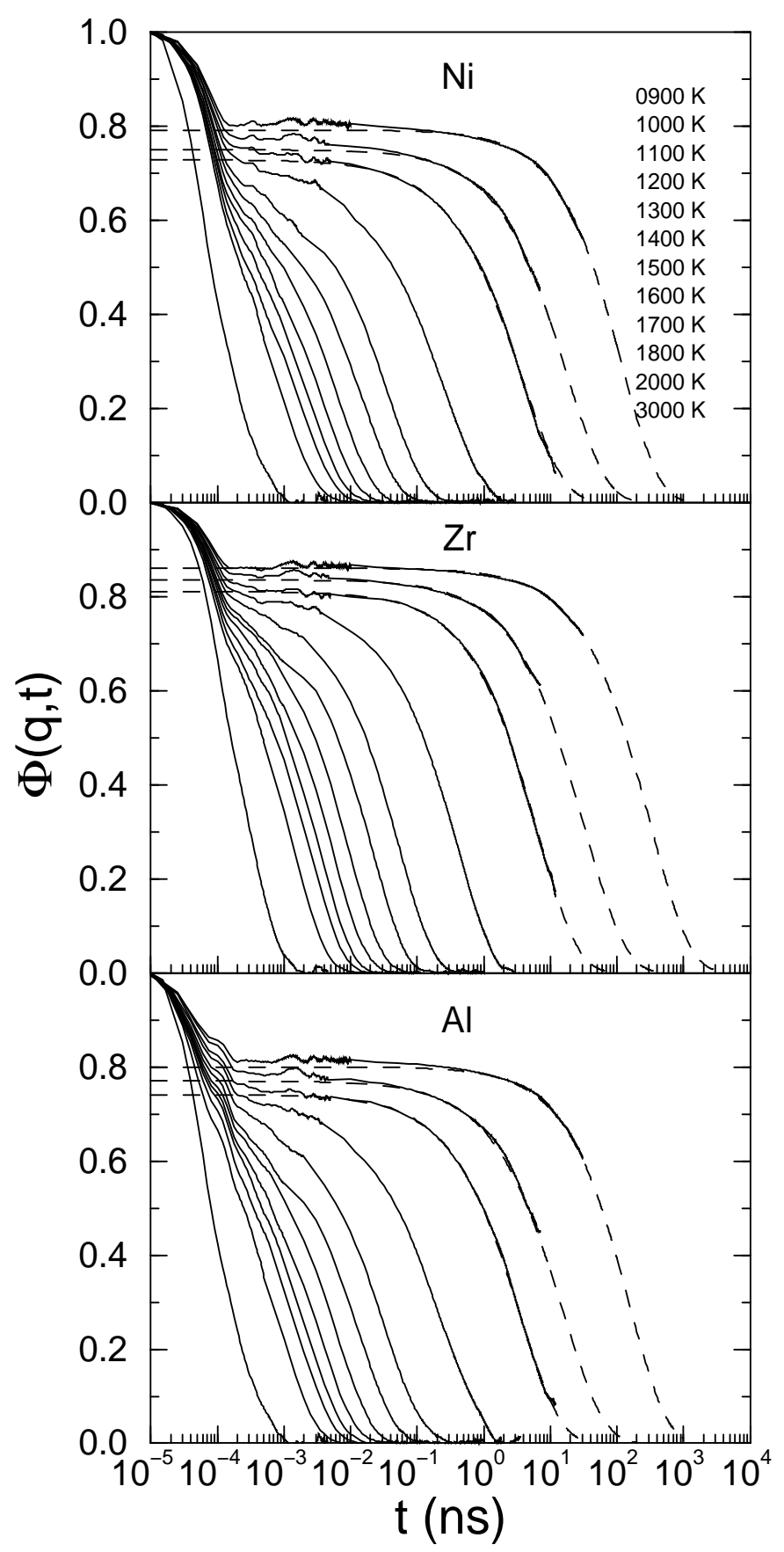

Figure 6.10: Time dependence of the self (or incoherent) intermediate scattering function $F_{s}(q, t)$ for different temperatures at the wave vector $q_{1}=2.24 \AA^{-1}$. The dotted lines are fits of the long-time decay whith a KWW law Eq. 6.13. 
to involve Fourier transform artefacts because of the finite size of our simulation box.

In Fig. 6.10 we show the time dependence (over nearly 7 decades) of the partial $F_{s}(q, t)$ for different temperatures and wave vector $q_{1}=2.24 \AA^{-1}$. This wavelength corresponds approximately to the location of the main peak in the total static structure factor.

We recognize the well known behavior of the density fluctuations decay. It takes place in one step at higher temperatures and in three steps in the undercooled liquid region, i.e., as $T_{c}$ is approached. The first step is a fast decay on the time scale of the vibrating atoms $(t<0.5 \mathrm{ps})$. This regime is associated with the vibration motion of an atom in the cage formed by its neighboring particles. The second step, the socalled $\beta$-relaxation regime, is identified by the upward curvature of the $F_{s}(q, t)$. The physical processes which take place in this regime are still a subject of debate in the research field of glasses, in particular, for metallic glasses. The $\beta$-regime is followed by a plateau (at intermediate temperatures it has the form of a shoulder) with a rapidly growing extension as the temperature approaches $T_{c}$. This behavior is very similar to that encountered in the time dependence of the MSD. It characterizes the transient trapping of a moving atom in the cage formed by its neighboring particles. The final step of the structural relaxations, the so-called $\alpha$-regime, is the region at larger times where $F_{s}(q, t)$ exhibits again a downward curvature. The $\alpha$-relaxation is associated with the final decay of the atomic arrangement. We see that for temperatures around $T_{g}$ the onset of the $\alpha$-process can be observed, but the correlator $F_{s}(q, t)$ does not decay to zero within the available observation time. This reflects that the latter is shorter than the $\alpha$-relaxation time scale. We note that a structural arrest below $T_{c}$ in the sense of the idealized MCT cannot be observed. It should manifest itself by a horizontal non decaying plateau of $F_{s}(q, t)$, not found in the temperature range investigated. This demonsrates once again that the simulated amorphous structure still is moving even below $T_{c}$ due to thermally activated hopping events not included in the idealized MCT.

The $\alpha$-relaxation is usually characterized by its relaxation time $\tau_{s}(T)$, whose value depends on the investigated variable and on the definition adopted to determine it. We will investigate here the relaxation time of the density fluctuations by using the conventional definition as the time after which the intermediate scattering function decays to $e^{-1}$ of its initial value. At those temperatures where $F_{s}(q, t)$ does not decay to this value, we deduced $\tau_{s}$ from a fitting of the long-time decay of the calculated curves whith a KWW law

$$
f(t) \propto \exp \left(-\left(t / \tau_{s} \beta\right), 1<\beta<0,\right.
$$

which has been found, by MD simulations [25, 27] and by experiments [86, 93], to describe well the long-time behaviour of the structural relaxations in undercooled 


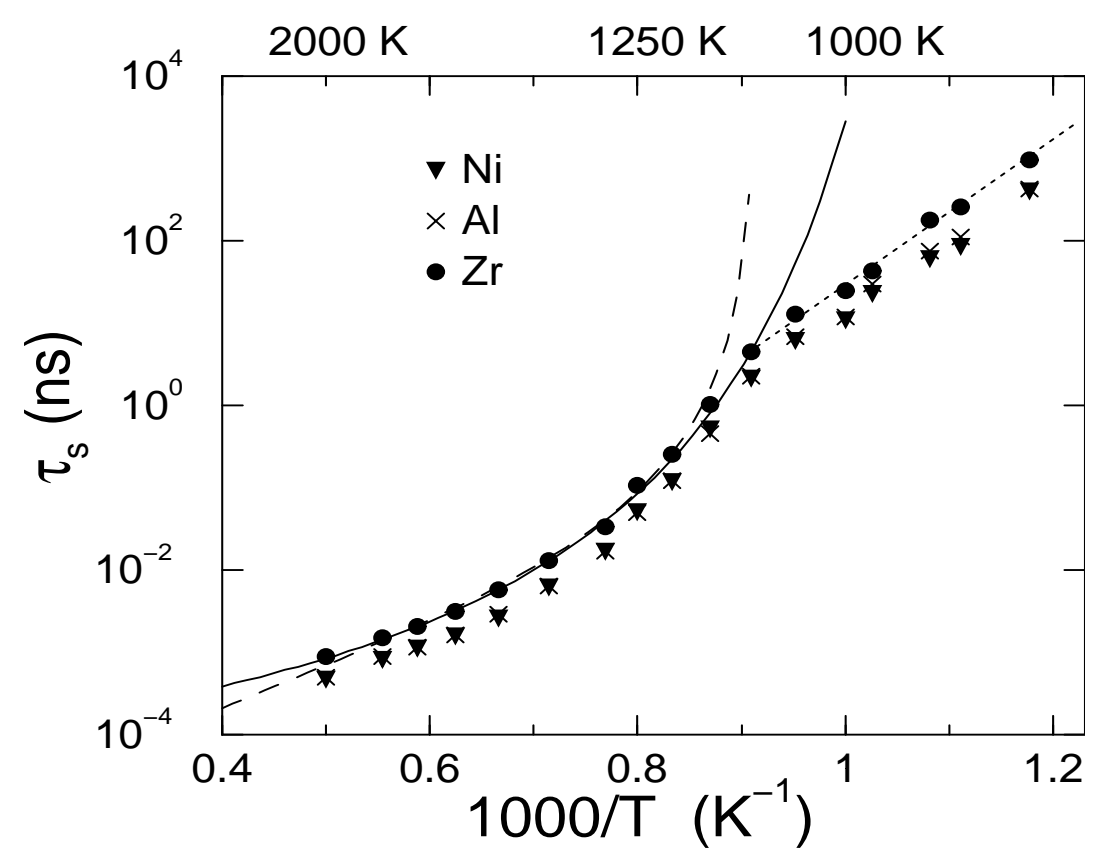

Figure 6.11: Arrhenius plot of the relaxation time $\tau_{s}$ from the decay of the intermediate scattering function. Note that $\mathrm{Ni}$ data coincide with $\mathrm{Al}$ ones on the whole temperature range investigated. Fits to Vogel-Fulcher law (solid line) and to a power law (dashed line) are illustrated for Zr data.

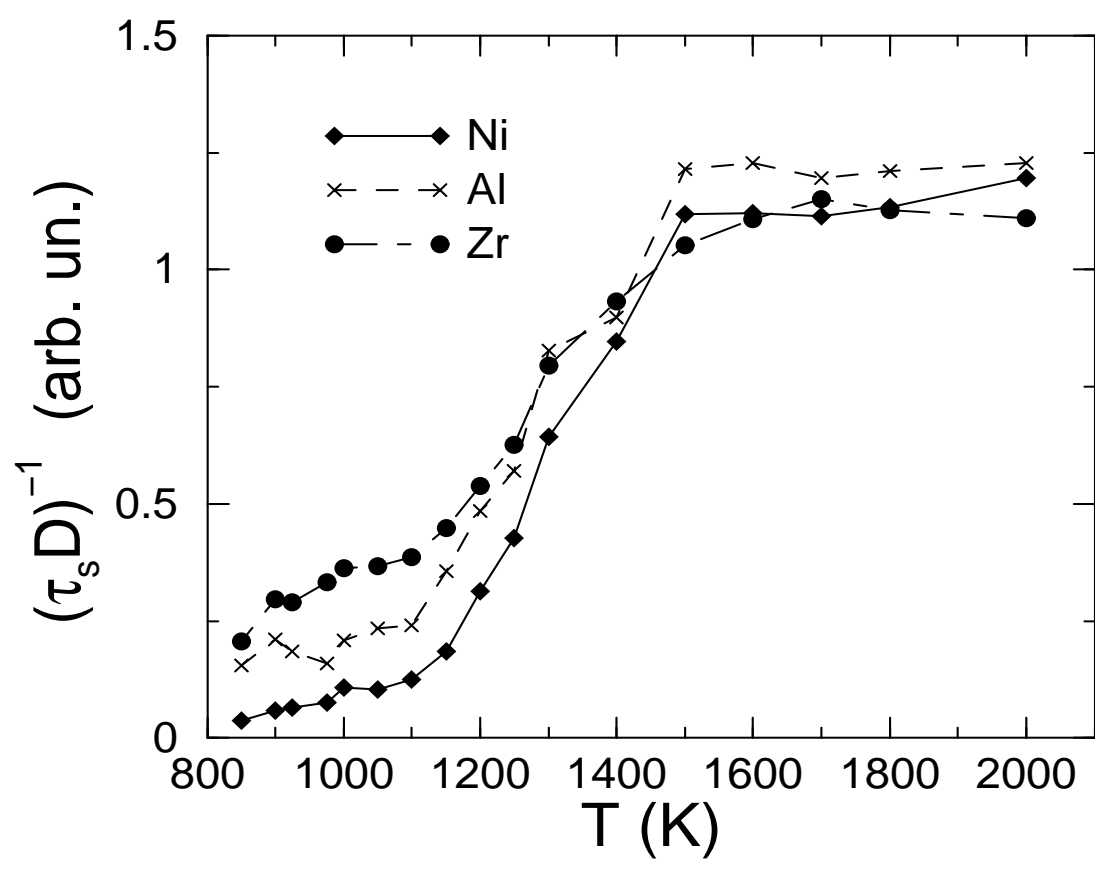

Figure 6.12: Temperature dependence of the products of the diffusion constants with the relaxation time $\tau_{s}$ 
liquids and glasses. The dashed lines in Fig. (6.10) additionally support this statement.

The temperature dependence of $\tau_{s}$ is presented in Fig. (6.11) for $\mathrm{Ni}, \mathrm{Zr}$ and $\mathrm{Al}$ at the wave vector $q_{1}$. At first sight, the behaviour of $\tau_{s}(T)$ is qualitatively very similar to that observed for the diffusion constants, especially a kink at $T_{g}$ followed by an Arrhenius behaviour at lower temperatures. A larger relaxation time for Zr atoms is consistent with their lower diffusivity due to their larger size and mass relatively to $\mathrm{Ni}$ and $\mathrm{Al}$ atoms.

The relaxation time $\tau_{s}$ exhibits, however, a particular behaviour which distinguishes it from the diffusivity, namely the parallel run of the three partial $\tau_{s}$ 's over the whole temperature range investigated. If we go back to the MSD in Fig. 6.1, we realize that $\tau_{s}$ corresponds to atomic displacement less than $2 \AA$. This means that the relaxation time $\tau_{s}$ at the main peak of the static structure factor describes structural relaxations dominated by small particles displacements only 94. And since these latter are governed by the viscous flow motion, it is therefore expected that $\tau_{s}$ at $q_{1}$ behaves similarly to the $D_{f}$ (Fig. $(6.9)$ ), i.e., the same temperature dependence and a parallel run for the three $\tau_{s}$ 's at higher as well as at lower temperatures.

In Fig. 6.11 we include a power-law and a Vogel-Fulcher fitting of the data obove $T_{g}$. The corresponding fit parameters are listed in tab.6.2. According to MCT [24], the relaxation time of the $\alpha$-relaxation in the supercooled liquids follows a power law dependence with the same exponent $\gamma$ and the same critical temperature $T_{c}$ as for the diffusion constants and the viscosity. $T_{c}$ and $\gamma$ being, moreover, independent of the wave vector $q$. In our case, $T_{c}$ from $\tau_{s}$ corresponds to that from the diffusion analysis within about $5 \%$. This is, however, not the case of $\gamma$, for which a deviation of $15 \%$ is observed.

Table 6.2: Parameters of the power law (after MCT) and Vogel-Fulcher fits for the relaxation times $\tau_{s}$ above $T_{g}$.

\begin{tabular}{ccccc}
\hline \hline & \multicolumn{2}{c}{$\mathrm{MCT}$} & \multicolumn{2}{c}{$\mathrm{VF}$} \\
\hline & $T_{c}[K]$ & $\gamma$ & $B[K]$ & $T_{0}[K]$ \\
\hline $\mathrm{Ni}$ & 1080 & 2.85 & 3050 & 827 \\
$\mathrm{Zr}$ & 1095 & 2.75 & 3100 & 830 \\
$\mathrm{Al}$ & 1080 & 2.85 & 3050 & 820 \\
\hline \hline
\end{tabular}

Fig. 6.12) displays the inverse product of the relaxation time $\tau_{s}$ and the diffusion constant $D_{\alpha}$ for each species, as function of the temperature. We observe that this product is constant at higher temperatures down to $T \approx 1500 \mathrm{~K}$, and then starts to decrease. The intuitive idea behind this plot is the following: $\tau_{s}$ describes the flowlike motion of the particles, while $D_{\alpha}$ describes flow as well as hopping diffusion. 
In the absence of hopping processes, we expect that $D_{\alpha}^{-1}$ and $\tau_{s}$ exhibit the same temperature dependence, their product must then be constant. This is indeed what we observe in the liquid state at higher temperatures. The decoupling of these two quantities at lower $\mathrm{T}$ might be taken as a hint for the onset of hopping processes. The temperature region where the decoupling occurs corresponds to that at which hopping diffusion has been identified from the qualitative analysis of the van Hove correlation functions.

\subsection{Shear stress fluctuations and shear viscosity}

Whereas the self-diffusion constant describes the single-particle motion, the viscosity represents a collective property, which involves many-particle correlations. The viscosity is the most used quantity to describe the development of rigidity in liquids. We have investigated the shear viscosity by means of the Green-Kubo relation [89]

$$
\begin{gathered}
\eta=\int_{0}^{+\infty} \eta(t) d t, \\
\eta(t)=\frac{1}{k_{B} T V}\left\langle\sigma^{x y}(t) \sigma^{x y}(0)\right\rangle .
\end{gathered}
$$

$\eta(t)$ is the stress autocorrelation function (SACF), a quantity that describes the decay of shear stress fluctuations. $\sigma^{x y}$ represents the off-diagonal elements of the macroscopic stress tensor given by

$$
\sigma^{k l}=\sum_{i=1}^{N}\left(m_{i} v_{i}^{k} v_{i}^{l}-\sum_{j>i}^{N} \frac{\partial \Phi\left(r_{i j}\right)}{\partial r_{i j}} \frac{r_{i j}^{k} r_{i j}^{l}}{r_{i j}}\right) \quad k, l=1,2,3,
$$

where $\mathbf{r}_{i}$ and $\mathbf{v}_{i}$ are the coordinates and the velocities, respectively, of the particle $\mathrm{i}, \mathbf{r}_{\mathbf{i j}}$ the vector joining particle $\mathrm{i}$ to particle $\mathbf{j}$, and $\mathbf{F}_{\mathbf{i j}}=-\partial \Phi\left(r_{i j}\right) / \partial \mathbf{r}_{\mathbf{i j}}$ the force exerted by particle $\mathrm{j}$ on particle $\mathrm{i}$.

The lack of averaging over the particles in Eq. (6.16) makes its statistical accuracy inferior to that of the diffusion constant. This has been compensated by averaging the value of the SACF over a large number of configurations (about $2 \times 10^{5}$ ) in addition to averaging over the three off-diagonal components of the stress tensor.

The Green-Kubo relation for the shear viscosity, Eq. (6.16), is related to the transverse-current autocorrelation function (TCAF) in its hydrodynamic behavior, i.e., in the long wavelength $(k \rightarrow 0)$ and low frequency $(\omega \rightarrow 0)$ limit [89]. The validity of this relationship supposes that the system is large enough to support this limiting behavior. Non-hydrodynamic behavior, with respect to the wavelength limit $(k \rightarrow 0)$, would manifest itself as finite-size effects in the calculated GreenKubo value of the shear viscosity. The direct identification of this behavior might be obtained by calculating the shear viscosity on a range of system sizes and to look 
for a size dependence. An alternative method is to determine the k-dependent shear viscosity $\eta(k)$ [95, 96] for wave vectors $\mathrm{k}$ larger than the value $k_{\text {min }}=2 \pi / L$, where $L$ corresponds to the simulation box length. $\eta(k)$ can be deduced from the long-time behavior of the TCAF in the frame of the generalized hydrodynamics formalism procedure. An extrapolation of $\eta(k)$ to the long wavelength limit $(k \rightarrow 0)$ then gives the shear viscosity $\eta$. By comparing this value with the one computed from the Green-Kubo relation, we might gain some insight into finite-size effects due to nonhydrodynamic behavior of the simulation. This treatment has, moreover, the advantage that it can be carried out within one and the same simulation. We have analyzed the $\mathrm{k}$-dependent viscosity for the binary liquid $\mathrm{Ni}_{35} \mathrm{Zr}_{65}$ system consisting of 648 particles. The results are given in appendix B. They show that the values of the shear viscosity determined by extrapolation are in agreement with those computed from the Green-Kubo relation. This provides support to the reliability of the GreenKubo relation for calculating the shear viscosity even for system with rather limited size. Unfortunately, this affirmation can be made only for higher temperatures above approximatly $1400 \mathrm{~K}$ because at temperatures below an extrapolation of $\eta(k)$ to the long wavelength limit becomes difficult due to the strong k-dependence of $\eta(k)$ in the vicinity of $k_{\text {min }}=2 \pi / L$. In order to determine possible finite-size effects in the temperature range around and eventually below $T_{c}$, the direct method, i.e. change of the system size, is required.

Fig. 6.13 displays the time dependence of the stress autocorrelation function normalized to unity for different temperatures. As in the case of the intermediate scattering function (section 6.4), we recognize the three steps characteristic of the structural relaxations: vibrational, $\beta$-, and $\alpha$-process. The SACF differs, however, by a significantly lower plateau at intermediate times. This reflects clearly the important contribution of vibrational motion to the shear stress decay.

At lower temperatures, where the SACF does not decay to zero within the computation time, we fitted the long-time decay of the curves with a KWW law. The latter describes the shear $\alpha$-relaxation as good as the density $\alpha$-relaxation behavior. The corresponding $\beta$ exponents are considerably smaller (in the range of 0.45 ). At these temperatures, the viscosity value has been evaluated by integrating this KWW-function.

The curve at $T=850 \mathrm{~K}$ exhibits a practically non decaying plateau within the available observation time $(20 \mathrm{~ns})$ and marks thereby the lower bound on temperatures at which our computer experiment is able to give an estimation of the shear viscosity. We note that even around $T_{c}$ it becomes quite difficult to give an accurate value of the viscosity, in view of the large fluctuations and the strong anisotropic behavior of the SACF. It is tempting to attribute this behavior to the fact that the system falls out of equilibrium continuously as $T_{c}$ is crossed. However, this explanation has to be considered with caution, since Böddecker [36] showed in a MD simulation of binary amorphous $\mathrm{Ni}_{50} \mathrm{Zr}_{50}$ that this behaviour also is found after an 


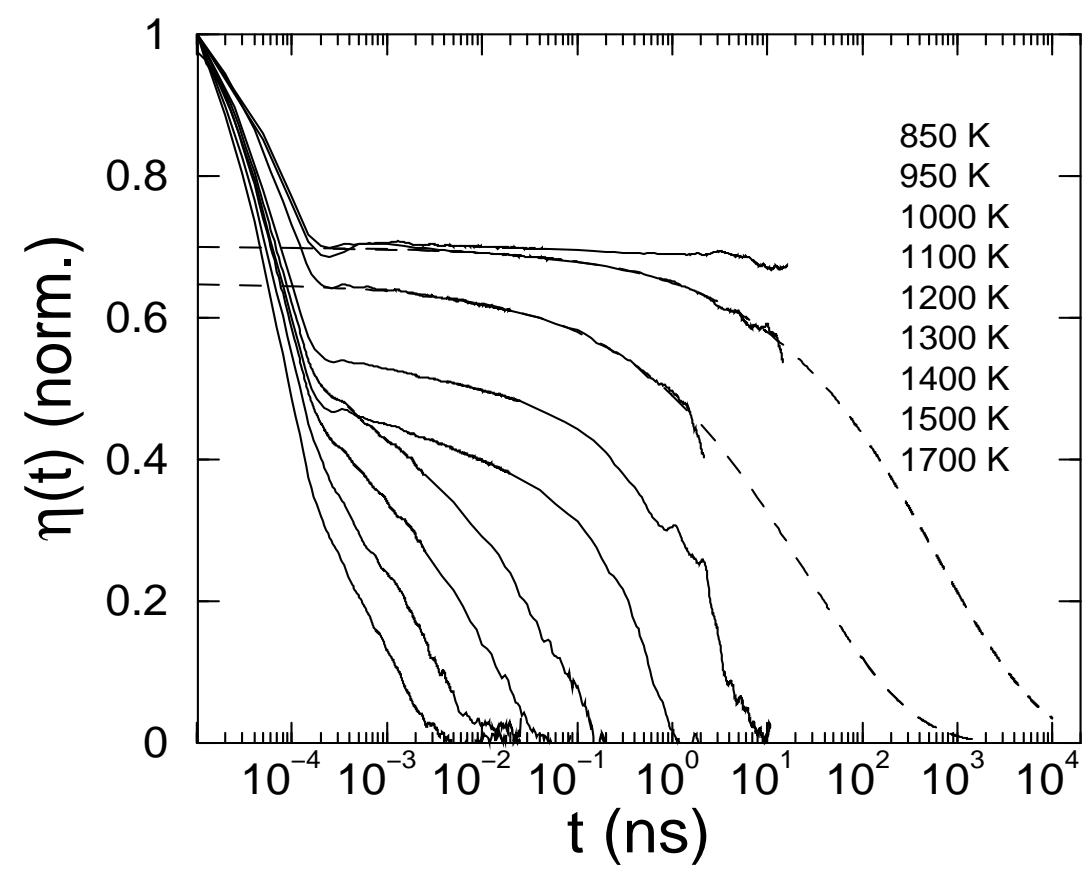

Figure 6.13: Time dependence of the shear stress autocorrelation function $\eta(t)$ (normalized to the unity) of $\mathrm{Ni}_{25} \mathrm{Zr}_{60} \mathrm{Al}_{15}$ for different temperatures. The dotted lines are fits of the long-time decay whith a KWW law (Eq. (6.13))

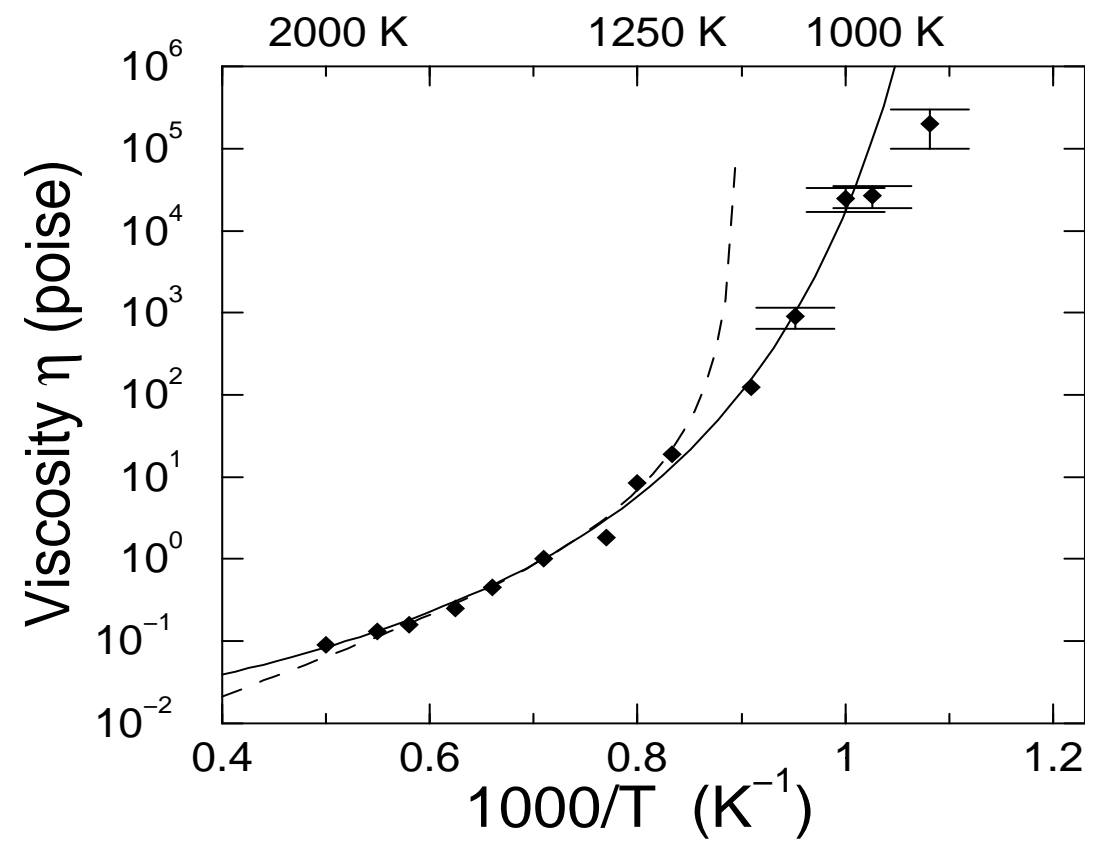

Figure 6.14: Arrhenius plot of the shear viscosity $\eta$ of MD simulated $\mathrm{Ni}_{25} \mathrm{Zr}_{60} \mathrm{Al}_{15}$. The solid line represents a fit of the hightemperature data obove $T_{g}$ with the Vogel-Fulcher law. The dashed line is a fit with the power law predicted by the MCT. 
observation time in the range of $1 \mu s$, that means a time comparable to the relaxation time of the system at the investigation temperature of $T=1050 \mathrm{~K} \approx T_{g}$. Finite-size effects would be another explanation of this behaviour. They could play a role in this temperature range because of the increasing rigidity of the system. This may give rise to shear stresses on length scales becoming comparable to the box length. Further simulations on systems of different sizes thus seem necessary to clearify this observation.

The temperature dependence of the viscosity is shown in Fig. (6.14). The values at lower temperatures are given along with their estimated uncertainities, which stem partly from the fitting procedure but mostly from the fluctuations mentioned above. We see that at high temperatures down to $T_{g}$ the viscosity exhibits a behaviour similar to that of the diffusion coefficient. Below $T_{g}$, the data are insufficient to identify an Arrhenius behavior, in contrast to the case of the diffusion constant and the density relaxation time. We have fitted the data with a power law as well as with a Vogel-Fulcher law. The parameters are listed in tab.(6.3). The critical temperature $T_{c}$ and the exponent $\gamma$ are very close to those found from the self-diffusion analysis, in agreement with the MCT predictions. The Vogel-Fulcher law fits significantly better the viscosity data than the power law, covering thereby a larger temperature range.

Table 6.3: Parameters of the power law (after MCT) and Vogel-Fulcher fits for the shear viscosity $\eta$ and the shear stress relaxation time $\tau$ above $T_{g}$.

\begin{tabular}{ccccc}
\hline \hline & \multicolumn{2}{c}{$\mathrm{MCT}$} & \multicolumn{2}{c}{$\mathrm{VF}$} \\
\hline & $T_{c}[K]$ & $\gamma$ & $B[K]$ & $T_{0}[K]$ \\
\hline$\eta$ & 1114 & 2.50 & 3200 & 785 \\
$\tau$ & 1100 & 2.48 & 3100 & 790 \\
\hline \hline
\end{tabular}

Instead by use of the viscosity, structural relaxations can be equivalently characterized by a specific relaxation time for the decay of the shear stress fluctuations in the long-time behavior. We define here a relaxation time $\tau$ as the time where the SACF $\eta(t)$ decays to a fraction of 0.2 of its initial value. We choose this arbitrary value so small (instead of the conventional value of $e^{-1}$ ) because the $\alpha$-relaxation of the shear mode starts at quite low values of the correlator $\eta(t)$ (lower than for the correlators $\left.F_{s}(q, t)\right)$, as it can be seen from the relatively low plateau position in Fig. 6.13). The temperature dependence of $\tau$ is displayed in Fig. 6.15 with the corresponding power law and Vogel-Fulcher fitting. The corresponding parameters are given in tab. 6.3.

The Vogel-Fulcher curve can be used to estimate the experimental glass tem- 


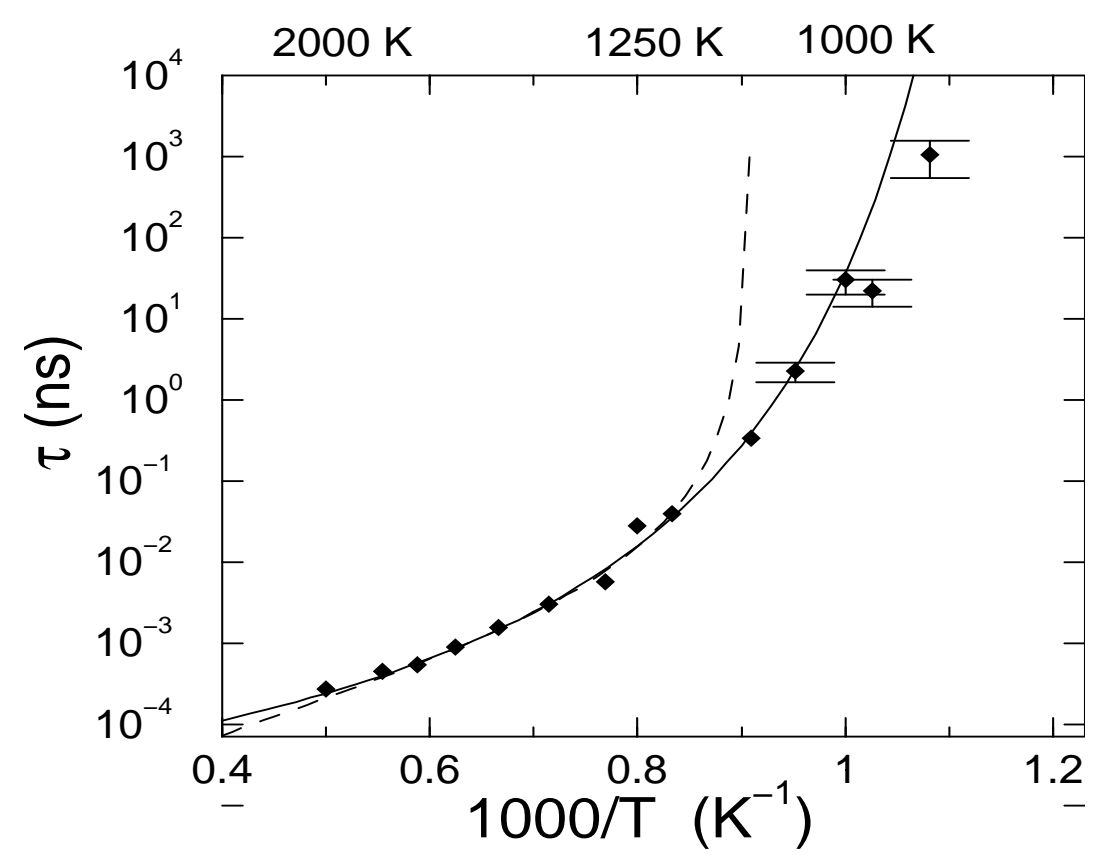

Figure 6.15: Arrhenius plot of the shear stress relaxation time $\tau$ of MD simulated $\mathrm{Ni}_{25} \mathrm{Zr}_{60} \mathrm{Al}_{15}$. The solid line represents a fit to the hightemperature data obove $T_{g}$ with the Vogel-Fulcher law. The dashed line is a fit with the power law.

perature ,i.e., the temperature at which the viscosity of the system reaches the conventional value of $10^{13}$ poise. This extrapolation gives a value of $850 \mathrm{~K}$ which is identical to the value deduced from the self-diffusion analysis in section 6.1. Extrapolation from the shear stress relaxation time $\tau$ to the macroscopic value of $10^{2} \mathrm{~s}$ leads to the same value. This shows once again that our model allows to reproduce the experimental, low-cooling-rate calorimetric glass temperature $\left(T_{g}^{e x p} \approx 720 \mathrm{~K}\right)$ within a precision of about $15 \%$.

\subsection{Stokes-Einstein relation}

The Stokes-Einstein (SE) relation was originally introduced to describe the diffusive motion of a large brownian particle in a continuous fluid with shear viscosity $\eta$ [89]

$$
d=\frac{k_{B} T}{c \pi \eta D}
$$

where $d$ is the "effective" diameter of the particle and c a constant depending on the assumptions made about the contact between the surface of the sphere and the velocity field of the fluid. If the "stick" boundary conditions are used, the fluid velocity at the surface matches with that of the particle and $c=3$. In the "slip" approach, the normal component of the fluid velocity is set equal to the normal component of the particle velocity with non tangential force acting on the sphere. 
In this case one gets $c=2$.

Although the SE relation was derived from purely macroscopic considerations, it is found to work surprisingly well in simple liquids on the atomic scale. Use of the slip boundaries leads generally to values of the effective diameter $d$ in reasonable agreement with the atomic diameters.

In order to check the validity range of the $\mathrm{SE}$ relation for our system, we plot in Fig. (6.16) the stokes diameter $d$ versus temperature. The diffusion coefficient has been averaged over the three species of the system and the "slip" boundary conditions has been assumed. From this figure we see that $d$ essentially is constant at higher temperatures with $d \approx 2.1 \AA$. This value underestimates somewhat the averaged atomic diameter of the system $(\approx 3 \AA)$. We note that it is possible to evaluate the SE relation for each species separately with the corresponding partial diffusion constants. This leads, however, to a diameter ratio $d_{Z r} / d_{N i} \approx 1.9$ which is markedly larger than the atomic size ratio of $\approx 1.3$.

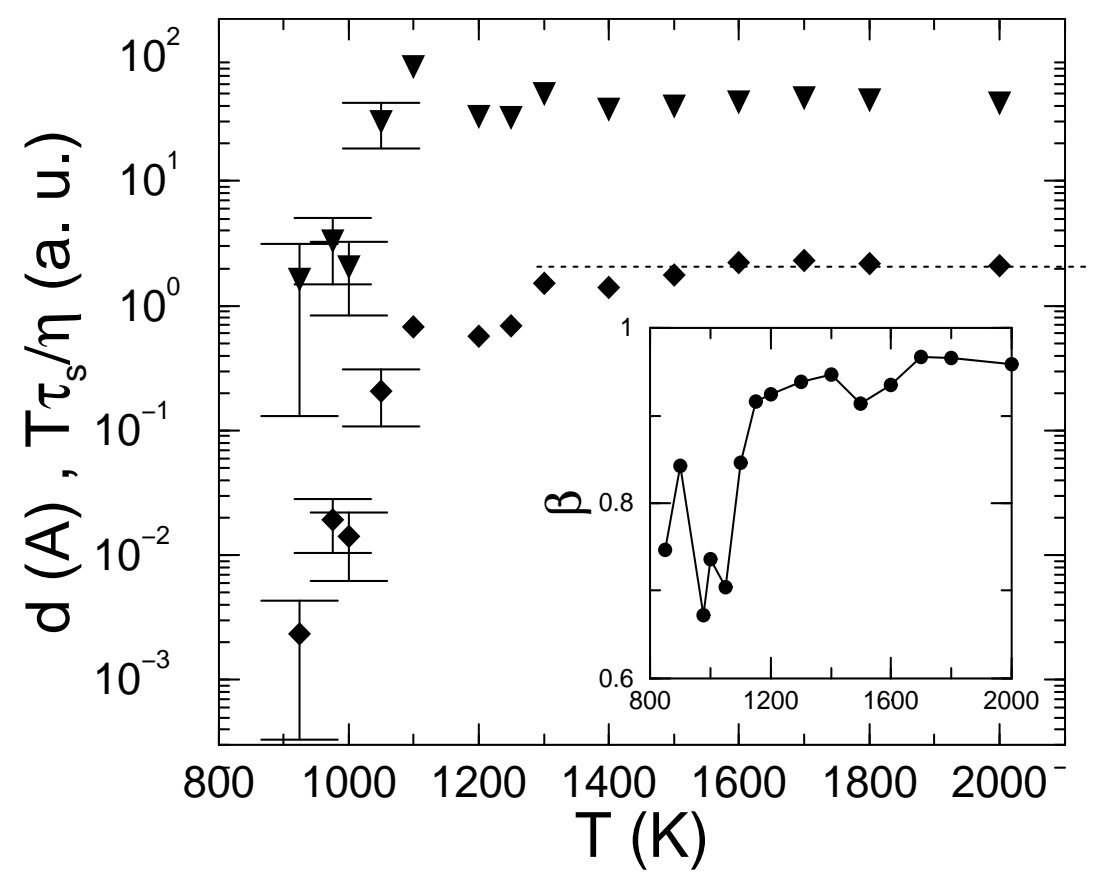

Figure 6.16: Temperature dependence of the Stokes diameter $d$ from Eq. 6.17) (diamonds) and of the products $T / \tau_{s} \eta$ (triangles). The inset shows the the KWW exponent $\beta$. The relaxation time $\tau_{s}$ and the exponent $\beta$ are determined from the decay of the total intermediate scattering function $F_{s}(q, t)$ averaged over the three species.

Around $T=1500 \mathrm{~K}$ the Stokes diameter starts to decrease, marking thereby the breaking of the SE relation. A decrease by about 2 decades is observed down to the GT temperature $T_{g}$. It is worth noticing that the failure of the SE relation occurs in the same temperature region where the onset of the activated hopping processes has 
been identified by means of the van Hove functions analysis. This might be taken as an indication that both effects are somehow correlated.

Note that the viscosity and the diffusivity start to decouple in the same temperature region where the diffusion constant and the density relaxation time $\tau_{s}$ manifest a decoupling (see section 6.4). The expression $T \tau_{s} / \eta$ (represented by triangles in Fig. 6.16) ) exhibits a value which is essentially independent of temperature down to $T_{g}$. The strong decrease of $T \tau_{s} / \eta$ below $T_{g}$ implies, however, that the glass transition affects differently $\tau_{s}$ and $\eta$. In the inset of the figure, we display the temperature dependence of the KWW exponent $\beta$ as determined from the decay of the total intermediate scattering function $F_{s}(q, t)$ averaged over the three species. A tendency of a constant $\beta$ value at high temperatures is observed, followed by a decrease below $1600 K$. The T-dependence thus is similar to that of the Stokes diameter.

Similar observations about decoupling behavior and breakdown of the SE relation have been reported from experiments [97, 98] and MD simulations [94, 99] for fragile as well as strong glass formers. In particular, the power-law behavior $D \propto \eta^{-n}$ with $n \approx 0.5-0.7$ has been observed at temperatures around $T_{g}$ [97, 98], which corresponds to a decrease of the Stokes diameter by two to three orders of magnitude.

\subsection{Diffusion and entropy}

Bei crossing $T_{g}$ with increasing temperature, the diffusion coefficients (Fig. (6.2)) exhibit an enhanced temperature dependence in the supercooled liquid compared to the glassy state. Interpreted in terms of the Arrhenius law, this signilizes a higher activaton energy $Q$ and a higher prefactor $D_{0}$ (for example, $Q \approx 2$. $\mathrm{eV}$ and $D_{0} \approx 10^{-1}$ for $\mathrm{Ni}$ in our ternary system). We note here that for all three species the diffusion coefficients can be well approximated by an Arrhenius behavior in a range of about $200 \mathrm{~K}$ above $T_{g}$, suggesting the existence of a temperature independant energy barrier distributions even in the viscous supercooled region. A change in the slope of the diffusivity in association with the GT is a feature observed in many experiments [99, 100] (albeit depending on the diffusing species). Geyer et al. [101] reported for the case of Be diffusion in $\mathrm{Zr}_{41.2} \mathrm{Ti}_{13.8} \mathrm{Ni}_{10} \mathrm{Cu}_{12.5} \mathrm{Be}_{22.5}$ an increase of a factor 3 for $Q$ and over 28 orders of magnitude for $D_{0}$, values which seem to be unphysically high to be explained simply in terms of an activation energy and activation entropy. Sharma and Faupel [102] pointed out that the activation energy in supercooled liquids must be regarded as an effective quantity only. It can be much larger than the activation energy for overcoming activation barriers, because any increase in temperature in this region is accompanied by important structural changes, which open new transition channels.

To rationalize the dramatic increase of $Q$ and $D_{0}$ at $T_{g}$, Geyer et al. [101] suggested a modified Arrhenius law by taking in account the increasing contribution of the configurational entropy obove $T_{g}$, 


$$
D(T)=D_{0} \exp \left(-\frac{Q}{k_{B} T}\right) \exp \left(\frac{N \Delta S(T)}{k_{B}}\right) .
$$

The environment ("the cage") around a diffusing atom is supposed to be similar to that in the glassy state, the prefactor $D_{0}$ and the migration enthalpy $Q$ are therefore considered to be the same as those determined below $T_{g}$. On the other hand, the continuous liquid-like rearrangement of the environment offers for a transition step a larger number of target configurations than in the glassy state, thus enhancing the diffusion. This is reflected in the additive entropy term $N \Delta S(T) . \Delta S(T)$ is the change of the configuration entropy per atom in the supercooled liquid relatively to the glassy state and $N$ the number of atoms involved in this cooperative behavior around a diffusing atom.

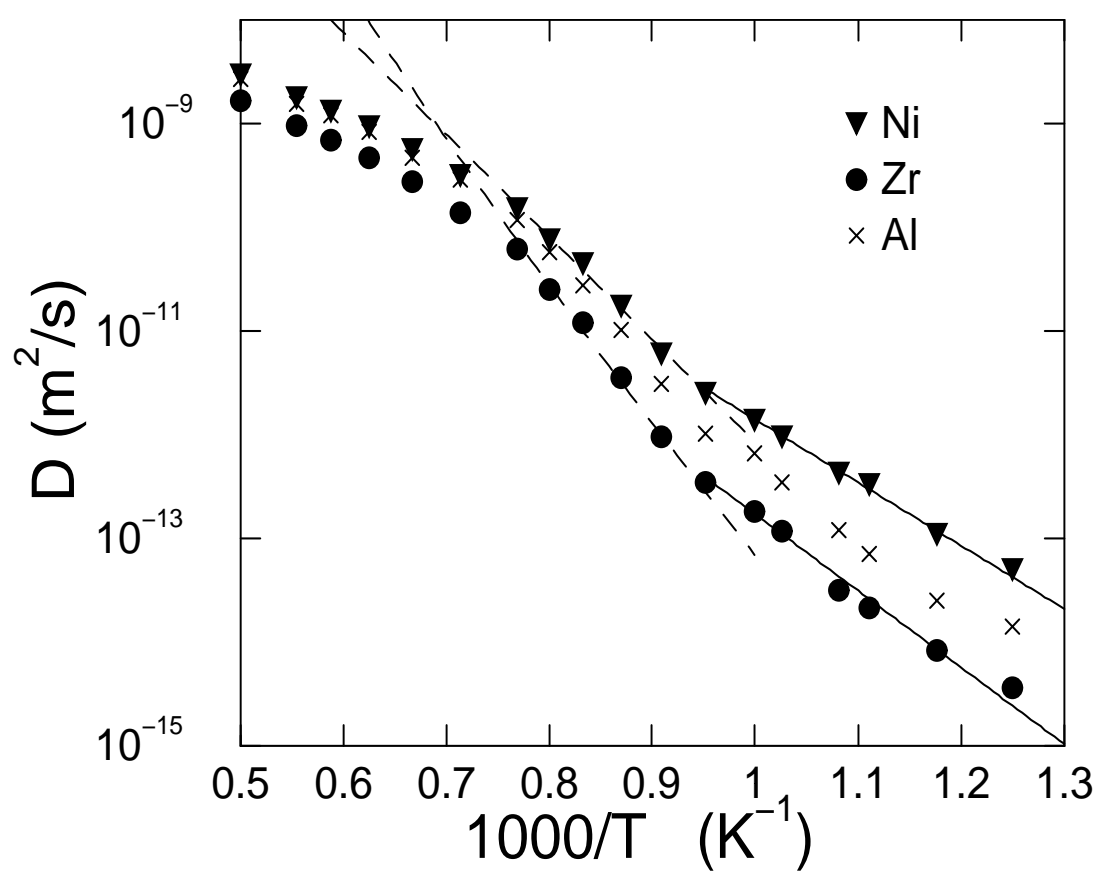

Figure 6.17: Diffusivity data with modified Arrhenius fit (dashed line) above $T_{g}$ according to Eq. 6.18). The solid lines are Arrhenius fits to the data below $T_{g}$.

$\Delta S(T)$ is calculated from the enthalpy data according to

$$
\Delta S(T)=\int_{T_{g}}^{T} \frac{\left(C_{p}^{S L}-C_{p}^{g}\right)}{T} d T .
$$

$C_{p}^{S L}$ and $C_{p}^{g}$ are the specific heat in the glassy state and the supercooled liquid, repectively.

Fig. (6.17) shows the fitting of our calculated diffusion data obove $T_{g}$ using Eq. 6.18. Note that $\mathrm{N}$ is the only fitting parameter here, while $Q$ and $D_{0}$ are those already determined below $T_{g}$. We obtain the values: $N_{N i}=9, N_{Z r}=13$, 
and $N_{A l}=10$, which are comparable with the corresponding average next-neighbors numbers: $z_{N i}=9.95, z_{Z r}=15.20, z_{A l}=12.05$ (see tab.(5.1).

The fitting fails at temperatures above $T \approx 1250 \mathrm{~K}$, which marks the limit of validity of Eq. 6.18) for our system. This may be an indication that thermal activation of individual transition events underlying the Arrhenius description of Eq. (6.18) becomes invalid in the undercooled melts and has to be substituted by the viscous-flow-like motion of the mode coupling picture [24]. On the other hand, the failure may be an indication that particular features of Eq. 6.18 have to be changed. E.g., the parameter $N$ that describes the length scale of the cooperative rearrangement is assumed here to be constant, while it is expected that the cooperativity is reduced with increasing free volume. A temperature dependent $N$ would be thus more appropriate.

Despite of the reasonable values of $N$ obtained by Geyer et al. [101] or those we obtained here, we have to mention that it is not obvious that the additive configurational entropy term in Eq. 6.18) directly determines the matter transport. Effects of the saddle point configuration may come into play, making plausible an additional proportionality factor.

\subsection{Comparison of dynamic properties in ternary $\mathrm{Ni}_{25} \mathrm{Zr}_{60} \mathrm{Al}_{15}$ and binary $\mathrm{Ni}_{35} \mathrm{Zr}_{65}$ alloys- Atomic- level stress tensor}

Fig. 6.19) and (6.18) compare the diffusivity and the viscosity, respectively, between the ternary $\mathrm{Ni}_{25} \mathrm{Zr}_{60} \mathrm{Al}_{15}$ and the binary $\mathrm{Ni}_{35} \mathrm{Zr}_{65}$ amorphous alloys. $\mathrm{Ni}$ and $\mathrm{Zr}$ diffusivities in the ternary system are higher by a about a factor 6 than in the binary one, which is also consistent with a lower viscosity in the first relatively to the second system.

The bend of the diffusion curves in the GT region occurs at slightly higher temperatures for the binary than for the ternary alloy. This is in agreement with a higher $T_{g}$ for the first relatively to the second system (see chapter 4). Note that the bend corresponds to approximately the same diffusivity value in both systems, which provides support to the kinetic nature of the glass transition in our simulations (the effective cooling rates in both cases being comparable).

Enhanced diffusivity is conventionally associated with the presence of a higher amount of free volume. This suggests, thus, that the free volume in the ternary system is larger than that in the binary system. The definition of the free volume in both systems, in order to check this last assumption, is however not an obvious question. Owing to the non-additive character of the pair-potentials, the particles cannot be treated here as simple hard spheres with defined radii as underlying the 


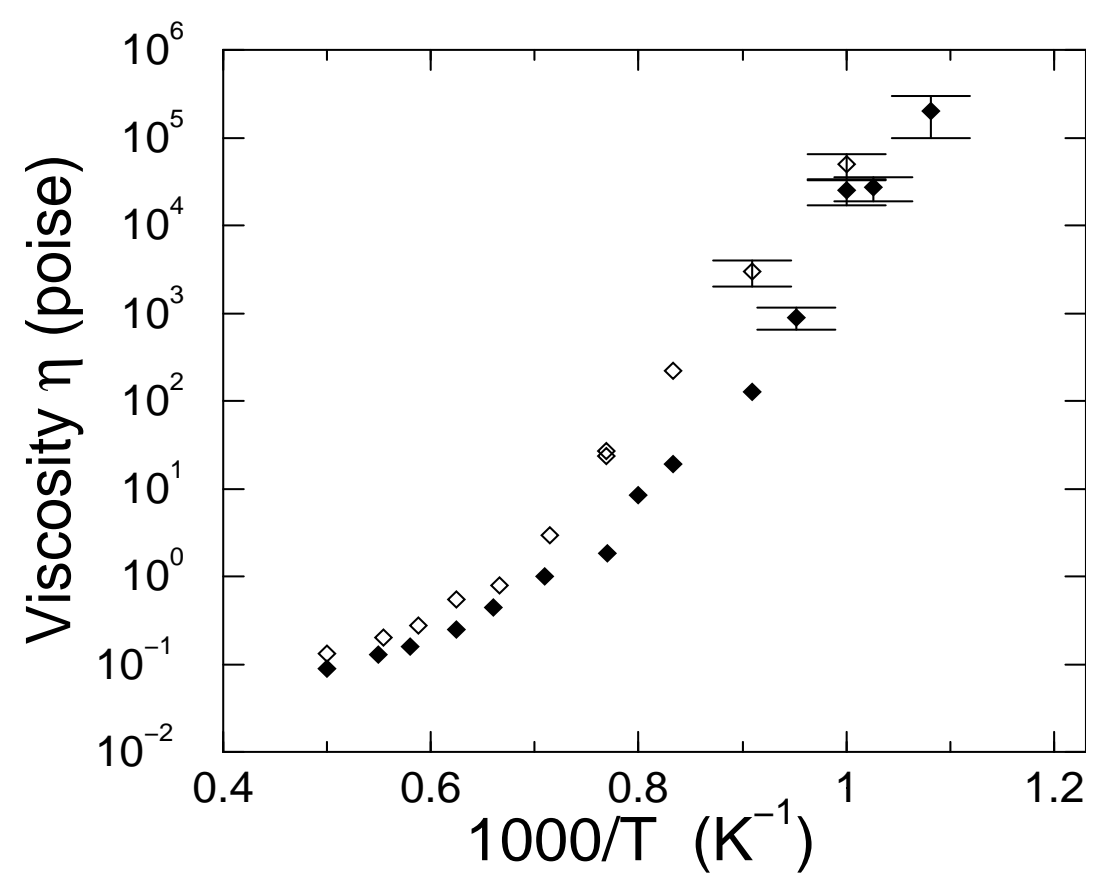

Figure 6.18: The shear viscosities of the ternary $\mathrm{Ni}_{25} \mathrm{Zr}_{60} \mathrm{Al}_{15}$ (full symbols) and the binary $\mathrm{Ni}_{35} \mathrm{Zr}_{65}$ (open symbols) alloys.

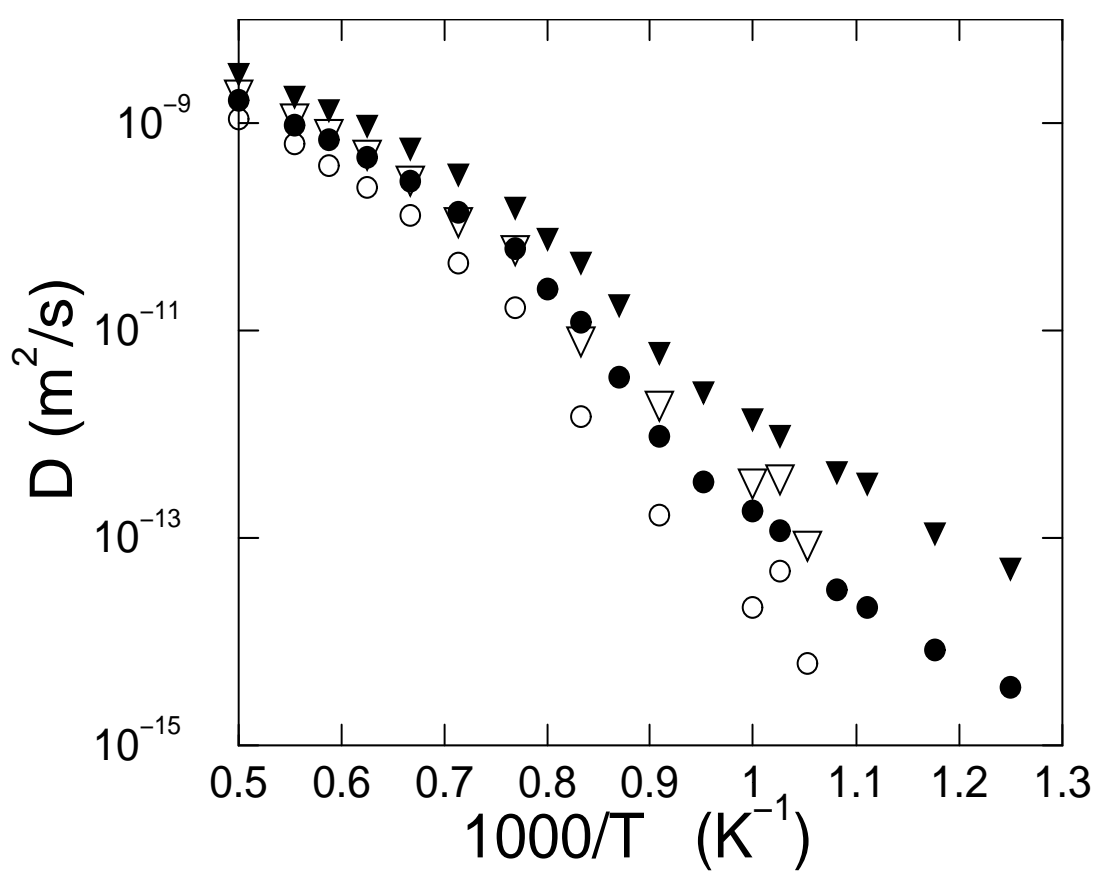

Figure 6.19: Self-diffusivity in the ternary $\mathrm{Ni}_{25} \mathrm{Zr}_{60} \mathrm{Al}_{15}$ (full symbols) and the binary $\mathrm{Ni}_{35} \mathrm{Zr}_{65}$ (open symbols) alloys. Triangles: nickel, circles: zircon. For $\mathrm{Al}$ in the ternary system, see Fig. 6.2 
simple free-volume picture.

An alternative approach to characterize the local atomic structure was introduced by Egami et al. [103]. They use the concept of structural defects in terms of the distribution of internal stresses on the atomic level. In the following we recall briefly this approach and apply it to our binary and ternary systems.

The stress tensor calculated at the position of an atom $i$ in a system of $\mathrm{N}$ atoms is given by 103 .

$$
\sigma_{i}^{k l}=\frac{1}{\Omega_{i}}\left(m_{i} v_{i}^{k} v_{i}^{l}+\frac{1}{2} \sum_{j \neq i}^{N} \frac{\partial \Phi\left(r_{i j}\right)}{\partial r_{i j}} \frac{r_{i j}^{k} r_{i j}^{l}}{r_{i j}}\right) \quad, k, l=1,2,3 .
$$

The quantities in this equation are the same as those in Eq. 6.16). The summation extends over all the atoms within the cut-off radii of the potentials. $\Omega_{i}$ is the local atomic volume which can be identified with the volume of Wigner-Seitz cell of atom i, approximated as 103

$$
\Omega_{i}^{-1 / 3}=\frac{\sum_{j \in n n} r_{i j}^{-1}}{2 \sum_{j \in n n} r_{i j}^{-2}},
$$

where the summation extends over the atoms within the nearest-neighbors (nn) shell of the atom $i$. In our case, we identify the nn shell with the volume of a sphere whose radius corresponds to the first minimum in the partial $\operatorname{RDF} g_{i j}(r)$.

The atomic pressure $p_{i}$ and the Von Mises shear stress $\tau_{i}$, which gives the magnitude of the shear stress at atomic level, (both are independent of the coordinate system) are defined as 121]

$$
\begin{gathered}
p_{i}=\frac{1}{3} \sum_{k=1}^{3} \sigma_{i}^{k k} \\
\tau_{i}=\left(\frac{1}{6}\left[\left(\sigma_{i}^{x x}-\sigma_{i}^{y y}\right)^{2}+\left(\sigma_{i}^{y y}-\sigma_{i}^{z z}\right)^{2}+\left(\sigma_{i}^{z z}-\sigma_{i}^{x x}\right)^{2}\right]+\left(\sigma_{i}^{x y}\right)^{2}+\left(\sigma_{i}^{y z}\right)^{2}+\left(\sigma_{i}^{z x}\right)^{2}\right)^{\frac{1}{2}}
\end{gathered}
$$

Egami et al. [103] relate the fluctuations in the local pressure $p_{i}$ with the fluctuations in the local density. They found a close correlation between $p_{i}$ and the atomic volume $\Omega_{i}$. On this basis, they introduce a simple concept of defects which they classified in two categories: n-type and p-type defects. The n-type defects are associated with sites of negative local pressure (tensile) and have lower-than-average densities, while p-type defects are related to sites with positive local pressure (compressive) and have higher-than-average densities. In crystalline solids, a n-type defect would correspond to a vacancy and a p-type defect to an interstitial.

The distributions for the atomic-level pressure $p_{i}$ and shear stress $\tau_{i}$ are displayed in Fig. 6.20 for each species in the binary and the ternary systems at $T=800 \mathrm{~K}$. As expected, the pressure distribution (averaged over all species) is centerered around the external hydrostatic pressure, held here constant (equilibrium 
conditions with $P=0$ ). The two distributions around $\mathrm{Ni}$ and $\mathrm{Zr}$ atoms show similar features in both systems. The pressure distribution around Ni is somewhat shifted to higher pressures with respect to the $\mathrm{Zr}$ distribution. According to the approach of Egami et al., this means that the Ni sites are associated preferently to p-type defects (interstitial-like) with higher-than-average densities. This observation provides support to the one in section 5.5 about the existence of a pronounced SRO in both amorphous systems and that $\mathrm{Ni}$ atoms reside in the centers of well-defined optimally packed structural units. Furthermore, the partial $\tau_{i}$ distributions of the ternary system are narrower with a slight shift towards lower shear stresses, which indicates that the ternary state is more relaxed than the binary one.

More striking is the large shift of the pressure distribution of Al to negative pressures as well as the shift of the shear-stress distribution of the same species to lower shear stresses. This feature confers rather a n-defect character to the $\mathrm{Al}$ sites, with lower-than-average densities (vacancy-like). It follows therefrom that by alloying $\mathrm{Al}$ to the binary $\mathrm{Ni}-\mathrm{Zr}$ alloy we introduce an additional free volume at the Al sites. That means Al looks like a smaller substitute of $\mathrm{Zr}$ at Zr-sites. This plausibly explains the enhanced diffusion, the lower viscosity and the more relaxed nature of the ternary $\mathrm{Ni}_{25} \mathrm{Zr}_{60} \mathrm{Al}_{15}$ relatively to the binary $\mathrm{Ni}_{35} \mathrm{Zr}_{65}$ amorphous alloy. 

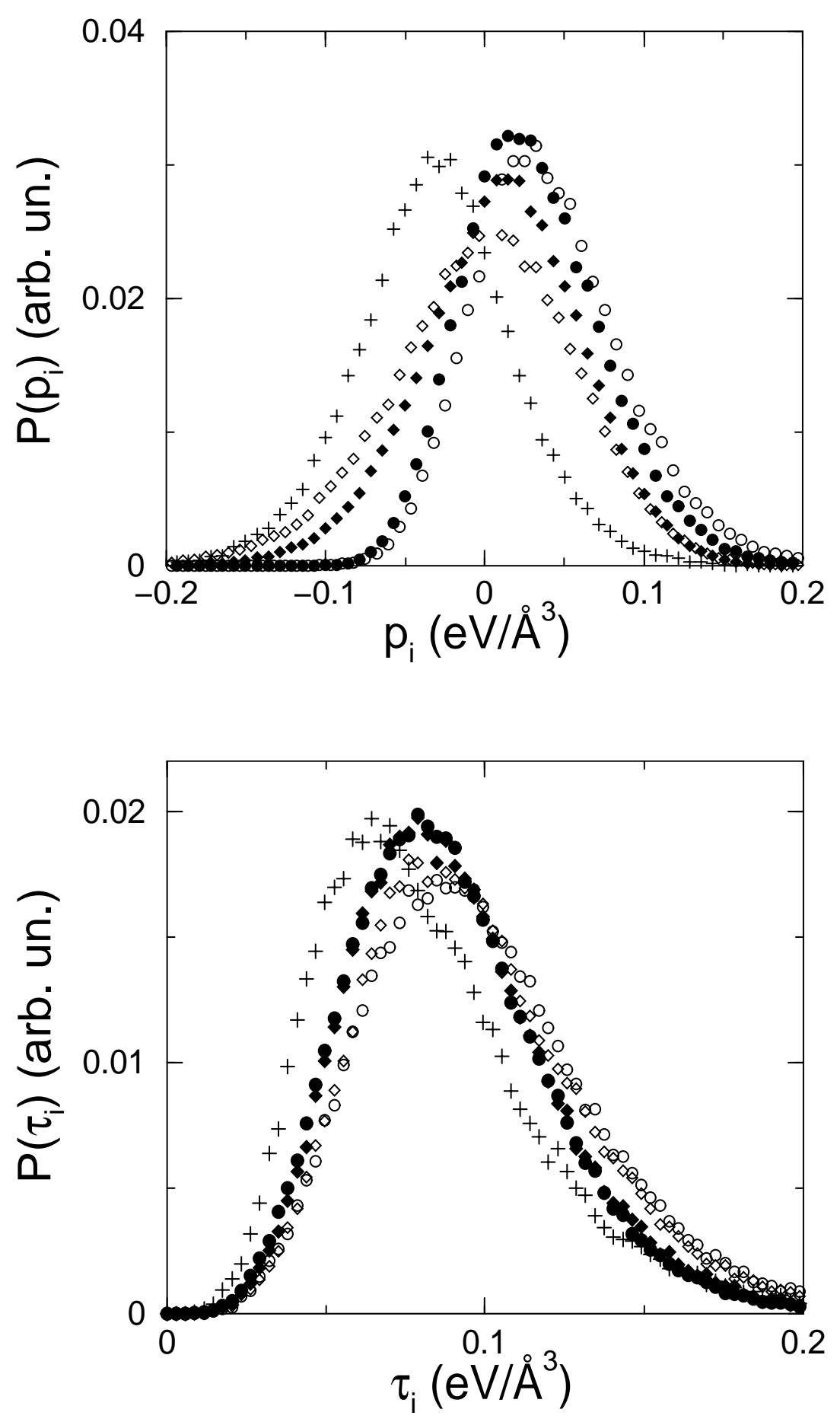

Figure 6.20: Distribution of the atomic level pressure (top) and von Mises shear stress (bottom) in the ternary $\mathrm{Ni}_{25} \mathrm{Zr}_{60} \mathrm{Al}_{15}$ (full symbols) and the binary $\mathrm{Ni}_{35} \mathrm{Zr}_{65}$ (open symbols) alloys at $T=800 \mathrm{~K}$. Circles: Ni; diamonds: Zr; plus: Al. 


\section{Chapter 7}

\section{Concluding discussion}

\subsection{Glass transition}

The experimental glass temperature $T_{g, \text { exp }}$ from DSC heating measurements for the binary $\mathrm{NiZr}_{2}$ and the ternary $\mathrm{Ni}_{25} \mathrm{Zr}_{60} \mathrm{Al}_{15}$ alloy are around $652 \mathrm{~K}$ [104] and $720 \mathrm{~K}$ [4, respectively. These values are well below the present findings from our model, $1200 \mathrm{~K}$ for the binary and $1025 \mathrm{~K}$ for the ternary system determined from the bend in the energy or the diffusivity data. This discrepancy can be partly attributed to the cooling rate dependence of $T_{g}$. The cooling rate used in our MD simulations $\left(\approx 10^{10} \mathrm{~K} / \mathrm{s}\right)$ lies at least four decades above typical cooling rates used in experiments $\left(<10^{6} \mathrm{~K} / \mathrm{s}\right)$ and about 8 to 10 decades above the heating rate of the DSC measurements $(\approx 10 \mathrm{~K} / \mathrm{s})$. From heating experiments on $\mathrm{Ni}_{34} \mathrm{Zr}_{66}$ (near the composition studied in this work), Lück et al. [105] deduced a raise of the glass temperature up to about $942 \mathrm{~K}$ for a heating rate of about $10^{12} \mathrm{~K}$. Furthermore, Teichler [15] established that the simulation model of $\mathrm{Ni}_{50} \mathrm{Zr}_{50}$ based on HausleitnerHafner potentials overestimates the melting temperature by about $21 \%$ relatively to the experimental one. The same overestimation may effect the estimated GT temperature $T_{g}$ (he obtained a comparable $T_{g}$ of about $1050 \mathrm{~K}$ ). All these aspects have to be taken into account when comparing the glass temperature $T_{g}$ from our MD simulations with the experimental $T_{g, e x p}$ from DSC measurements. Moreover, there is a good agreement with less than $15 \%$ deviation between the experimental $T_{g, \exp }$ and our value $T_{g, e x t}=840 \mathrm{~K}$ determined by extrapolating the viscosity (or the diffusion) data of the ternary alloy to values typical for the experimental GT temperature. The data used for this extrapolation belong to the temperature region where the system is in equilibrium. Therefore the extrapolated value $T_{g, e x t}$ should be independent of the cooling rate.

The model we used in this work is thus able to reproduce the structural features of $\mathrm{Ni}_{25} \mathrm{Zr}_{60} \mathrm{Al}_{15}$ in a reasonable way, as seen in section 5.3, and also to predict with good accuracy the experimental GT temperature, the latter belonging to the significant dynamical features of a glassy system. 


\subsection{Prepeaks and medium-range Order}

Medium-range order (MRO) in liquids and amorphous solids is a feature that attracted an increasing interest recently. MRO refers to the existence of structural ordering occuring over range distances larger than the average nearest-neighbor distance, and giving rise to the so-called first sharp diffraction peak (FSDP) (or the prepeak) in the structure factor $\mathrm{S}(\mathrm{Q})$ at a $\mathrm{Q}$ value smaller than that of the main peak. The structural analysis of $\mathrm{Ni}_{25} \mathrm{Zr}_{60} \mathrm{Al}_{15}$ in section 5.6 reveals the existence of well-defined prepeaks in the partial Faber-Ziman structure factors $S_{N i N i}^{F Z}(q)$ and $S_{A l A l}^{F Z}(q)$. This is a clear signature that ordering beyond the nearest-neighbor distance is present in the studied system.

The structural origin of the FSDP remains a subject of a controversial discussion. In the case of network-forming glasses, known as "strong" glasses according to the classification given by Angell [68], the FSDP is attributed to the network structure due to the directional covalent bonding or the ionic polarization effects [78]. In a MD simulation of the network-forming system $\mathrm{SiO}_{2}$, Horbach et al. [78] pointed out that the microscopic explanation of the FSDP in this system is the tetrahedral-like structure and that the location of this prepeak is related to the distance between two neighboring tetrahedra. The FSDP has been also observed in some "fragile" glasses [16, 64, 92, 126]. In this case the MRO is attributed to the formation of structural units constitued of unlike atoms (good mixing behavior) or like atoms (demixing tendency). Notice that the MRO in this case could be dictated by steric conditions (topological range order) or by considerations of chemical affinities between the different atoms (chemical range order). These two orders are not necessarily independent of each other.

The ternary system $\mathrm{Ni}_{25} \mathrm{Zr}_{60} \mathrm{Al}_{15}$ simulated here is interesting from a more basic point of view as a model where two FSDPs of different nature are simultaneously present. This sheds some light on the problem concerning the physical origin of the prepeak in liquids and amorphous systems.

The Ni-prepeak at $q \approx 1.9 \AA^{-1}$ is found to be associated with the weak Ni$\mathrm{Ni}$ coordination in favour of the strong Ni-Zr heterocoordination, because of the good mixing behavior in the binary system Ni-Zr. The corresponding length of $\approx 3.9 \AA$ is related to the second nearest $\mathrm{Ni}-\mathrm{Ni}$ pair correlation. In the picture of the trigonal prismatic structural ordering (section 5.5), this length is related to an average distance between neighboring trigonal prisms, where the Ni atoms occupy the centres of these prisms. This interpretation has been suggested by Suzuki et al. [126] to explain the origin of the prepeak in the metal-metalloid amorphous alloys $\mathrm{Pd}-\mathrm{Ge}$ and Pd-Si. The authors recognize the MRO as the connectivity between adjacent trigonal prismatic units.

The Al-prepeak is found to be associated with the tendency of Al to cluster and to form chain-like structures, which seems to be induced by chemical affinities in 
the ternary system. The location of the prepeak $\left(q \approx 1.3 \AA^{-1}\right)$ is associated in this case with the distance between two $\mathrm{Al}$ clusters, and thus reflects a different type of MRO than in the Ni subsystem.

\subsection{Change in diffusion mechanism and decou- pling behaviour}

The analysis of the van Hove correlation functions in section 6.2 and the study of section 6.3 has shown that the onset of hopping processes actually takes place at a temperature of about $1400 \mathrm{~K}$, well above the dynamic GT temperature, $T_{c}$. In this temperature range the transport mechanism seems to change from a flowlike motion to a hopping-like one. Moreover, we have shown that this transition concerns primarily $\mathrm{Ni}$ and $\mathrm{Al}$. For $\mathrm{Zr}$ atoms, the flow-like motion seems to be the dominant transport mode even in the region of the kinetic GT and below. The van Hove distinct correlation function of $\mathrm{Zr}$ exhibits a behavior similar to that of normal liquids, while the same functions for $\mathrm{Ni}$ and $\mathrm{Al}$ develop a pronounced correlation peak at the origin $(r=0)$. The change of the transport mechanism around $T_{c}$ is a feature that has been confirmed by several MD simulation [28, 29, 30]. Our study suggests that there is a distinction between the behaviour of small and large particles.

While the occurence of hopping processes may be seen as a precursor of the dynamical Glass transition ( in the sense of MCT) it seems quite clear that they cannot be considered as a particular feature of the calorimetric GT. The latter is rather a matter of competing time scales, that is the time scale of the dynamics and that of the observation time. This point of view is supported by the fact that the onset of the hopping processes takes place already well above $T_{c}(T>1400 \mathrm{~K})$, while the experimental calorimetric glass temperature is as low as $720 \mathrm{~K}$. (This value is reproduced by our model within a deviation of $15 \%$, as mentioned in the preceding section).

The onset of hopping processes seems to be related to decoupling effects which we observe in the same temperature range, decoupling of the diffusivity from the density relaxation time, on one hand, and diffusivity from viscosity (or the failure of the Einstein-Stokes relation), on the other hand. Moreover, a kink in the temperature dependence of the cell volume has been observed at the temperature where these decouplings start to take place (chapter 4). Although there is evidence that these effects are related to each other, the nature of this relation is not well understood. In order to clear this relation further investigated still are necessary.

In a two dimensional study of a binary mixture of soft disks, Perrera et al. [94] also observed the decoupling between the self-diffusion and the density relaxation time (at the first peak of the structure factor) below a crossover temperature. 
They argued that this decoupling arises from the increasing transient localization ("caging") effect as the system is cooled. The transition from ballistic to diffusive dynamics gives rise to larger changes in the temperature dependence of the relaxation time, which occurs on (short) length scales comparable to that transition. On the other hand, this transition length is insignificant when compared to the length scale over which the self-diffusion constant is defined. In section 6.3 we used a similar arguing to explain the same decoupling. The relaxation time $\tau_{s}$ of the density fluctuations at the first peak of the structure factor is associated with the flow motion of the particles. In this way, the diffusivity and relaxation time $\tau_{s}$ have the same time dependence as long as the hopping processes are negligible.

In an analysis of experimental data of viscosity and diffusion of several fragile glasses, Rössler [97] reports that the breakdown of the Stokes-Einstein relation occurs at a particular temperature, which he identifies with the kinetic GT temperature $T_{c}$ of the MCT. Rössler associates the breakdown with a change of the diffusion mechanism in the supercooled liquid. Our system gives evidence that the failure of the SE relation may occur at as high temperatures as $T \approx 1.4 T_{c}$. The ratio of $T_{c}$ to the temperature where the SE relation fails may depend on the system studied and particularly on its fragility.

\subsection{Heterogeneity of the diffusion}

Many authors [94, 97, 106, 107, 108] have attributed the failure of the Stokes-Einstein relation and the decoupling behavior observed in the supercooled liquid of glassy systems to the existence of a spacial heterogeneity in the distribution of local relaxation times, in other words to the heterogeneous coexistence of different regions with different mobilities. It is proposed that the different physical quantities averaged to obtain transport and relaxation time scales sample different parts of the heterogeneity distribution and, hence, exhibit different temperature variations. According to this interpretation, the faster increase of the diffusivity with respect to the viscosity in the undercooled liquid is explained by the fact that the increase of the mean-square displacement is dominated by the more mobile regions ("soft channels"), while the decay of the stress correlation function is dominated by the slower rigid regions. The difference between the different averages increases more and more as the distribution broadens upon cooling.

This point of view is in agreement with the increasing non-exponential (nonDebye) relaxation behavior inherent to undercooled liquids with decreasing temperature [86]. One microscopic scenario that has been proposed to explain this feature is the superposition of different exponential contributions with different relaxation rates [109]. Furthermore, the KWW exponent $\beta$, which is a measure of this nonexponentiality, is found [110] to decrease as the temperature is lowered toward the glass temperature, indicating the broadening of the distribution of the relaxation 
rates. In section 6.4 and 6.5, we have, indeed, observed such a non-exponential behavior in the long-time decay of the intermediate scattering function and of the shear stress autocorrelation function as well as a decrease of the $\beta$ exponent on cooling.

A number of recent experiments have given evidence of the non-homogeneous nature of relaxation processes in amorphous structures. By selective photobleaching of probe molecules in supercooled ortho-terphenyl, Cicerone and Ediger [106], for instance, established the existence of long-lived clusters with different mobilities. The direct characterization of the size and shape of such local kinetic domains and of the microscopic mechanism reponsible for this phenomenon remains a difficult experimental task. Here MD simulation may turn out to be a powerful alternative tool to gain more insight in this phenomenon. Donati et al. [111] used a LennardJones model to show that stringlike cooperative molecules motions are present even at temperatures well above the kinetic GT temperature $T_{c}$. The mean length of these strings is found to increase upon cooling. Similar observations have been reported by other MD studies [112].

A systematic study of dynamical heterogeneities has been not attempted in the present work. Neverthless, we think that there is more than one indication of their existence in the systems investigated here. As discussed in the context of the van Hove functions analysis in section 6.2, the onset of hopping processes and the onset of correlated motions at high temperatures well obove $T_{c}(T>1400 \mathrm{~K})$ are one indication. These phenomena can be attributed to the fact that stable optimally packed regions develop in the liquid phase making possible these jumps and correlated motions. This may also explain why the onset of hopping processes and the failure of the Stokes-Einstein relation are observed in the same temperature region.

The idea of dynamical heterogeneities in form of long-living clusters of particles and the growth of the associated length scale is reminiscent of the concept of "cooperatively rearranged regions" put forward by Adam and Gibbs [113] to describe the dramatic change of transport properties of supercooled liquids upon cooling. They proposed that viscous flow occurs via increasingly cooperative rearrangement of particles groups.

Huge effort have been made to identify this cooperativity experimentally. Heesemann et al. [81] investigated the isotop effect of cobalt diffusion in the amorphous state of several conventional metallic glasses (e.g. Co-rich Co-Zr alloys) as well as in the deeply supercooled melt of the bulk glass forming alloy $\mathrm{Zr}_{41.2} \mathrm{Ti}_{13.8} \mathrm{Ni}_{10} \mathrm{Cu}_{12.5} \mathrm{Be}_{22.5}$. From the very small value of the isotop effect, they concluded that Co diffusion in these alloys is a cooperative process involving about ten atoms. The same research group [32] confirmed this statement by measuring the pressure dependence of Co diffusion in these alloys where an almost vanishing activation volume was found. Geyer et al. [101] used an approach based on the change of the configurational entropy at the glass temperature (section 6.7) to show that 
the number of atoms involved in the cooperative motion around a diffusing particle is comparable with the number of the nearest-neighbors. Our results in section 6.7 are in agreement with the prediction of this approach.

In a MD simulation of amorphous $\mathrm{Ni}_{1-\mathrm{x}} \mathrm{Zr}_{\mathrm{x}}$ alloys, Teichler 30] established that in the Zr-poor structure, about $80 \%$ of $\mathrm{Ni}$ transition take place via cooperative processes, where a few $\mathrm{Ni}$ atoms are involved. This cooperativity appears in form of ring or chain processes. Similar observations have been presented by Gaukel et al. [91, 92 in a MD simulation of the glassy system $\mathrm{Cu}_{33} \mathrm{Zr}_{67}$. Furthermore, the latter authors associate the highly cooperative hoppings in the glassy state to the well-known low frequency localized vibration modes.

\subsection{Glass forming ability}

Busch et al. [19] have carried out a comparative study of a number of eutectic, mainly Zr-based glass forming systems. They have studied thermodynamics and kinetics of supercooled liquids by measuring the temperature dependence of the thermodynamic functions and the viscosity. In order to explain the large glass forming ability of bulk metallic glasses compared to the conventional ones, Busch et al. advanced kinetic and thermodynamic arguments.

Regarding the kinetics, Busch et al. deduced that bulk metallic glasses exhibit a behavior which is close to that of the strong glass forming silicate melts like $\mathrm{SiO}_{2}$, namely a relatively weak temperature dependence of the viscosity close to the glass transition. This strong liquid nature implies unusually high viscosity values of the melt. In the case of $\mathrm{Zr}_{41.2} \mathrm{Ti}_{13.8} \mathrm{Ni}_{10} \mathrm{Cu}_{12.5} \mathrm{Be}_{22.5}$ and $\mathrm{Mg}_{65} \mathrm{Cu}_{25} \mathrm{Y}_{10}$, viscosities around the melting temperatures of the order of 50 poise have been measured. This gives evidence that the bulk metallic glasses are much more viscous than pure metals or some binary alloys, where viscosities of the order of $10^{-2}$ poise are observed at the melting point. The high viscosity of the melt is considered as a crucial factor in understanding the superior glass forming ability of bulk glasses, since it implies sluggish kinetics in the entire range of the supercooled liquid leading to a low nucleation and growth rate for crystallization.

According to these kinetic arguments, the expected large glass forming ability of the ternary $\mathrm{Ni}_{25} \mathrm{Zr}_{60} \mathrm{Al}_{15}$ alloy compared to the binary $\mathrm{Ni}_{35} \mathrm{Zr}_{65}$ reflects a more sluggish kinetics in the first alloy with respect to the second. At first sight, this condition seems to be not fulfilled in our case, since the kinetic comparison in section 6.8 shows that the dissolution of $\mathrm{Al}$ into the binary alloy $\mathrm{Ni}-\mathrm{Zr}$ leads to an increased diffusivity and decreased viscosity for a given temperature. However, with regard to the glass forming ability, a comparison of the kinetics in two alloys makes sense only if these alloys are considered at their respective melting points. The experimental values of the melting points are around $1400 \mathrm{~K}$ for $\mathrm{Ni}_{35} \mathrm{Zr}_{65}$ and about $1200 \mathrm{~K}$ for $\mathrm{Ni}_{25} \mathrm{Zr}_{60} \mathrm{Al}_{15}$. Note that the ratio of the melting points is in agreement with the ratio 
of the corresponding glass temperatures, both reflecting the higher binding strentgh of Zr-Ni relatively to Zr-Al. Using the data of Fig. 6.18), we get melt viscosities of $\approx 25$ poise for $\mathrm{Ni}_{25} \mathrm{Zr}_{60} \mathrm{Al}_{15}$ and $\approx 3$ poise for $\mathrm{Ni}_{35} \mathrm{Zr}_{65}$. We conclude therefrom that the ternary sytem exhibits a more sluggish kinetics at its melting point than the binary one, which is in agreement with the (experimentally) observed larger glass forming ability of the first alloy relatively to the second.

Busch et al. [19] have also put forward thermodynamic arguments to describe the large glass forming ability of bulk metallic glasses. They established that glass formers with the lowest critical cooling rates have smaller Gibbs free energy differences $\Delta G_{l-x}$ between the undercooled liquid and the corresponding crystalline mixture, i.e. smaller driving forces for cristallization. Bulk glass formers are thus thermodynamically closer to the crystalline state compared to conventional glass formers. This originates mainly from a smaller entropy of fusion, which determines the slope of $\Delta G_{l-x}$ at the melting point. This suggests that the melts of bulk metallic glass formers have a tendency to develop a pronounced chemical short range order even around the melting point. Atom probe field microscopy and small angle neutron scattering experiments have confirmed the existence of such chemical SRO [20].

A direct verification of the just mentioned thermodynamic arguments for our ternary system $\mathrm{Ni}_{25} \mathrm{Zr}_{60} \mathrm{Al}_{15}$ with respect to the binary $\mathrm{Ni}_{35} \mathrm{Zr}_{65}$ alloy is not obvious, since there is not sufficient experimental informations about the crystalline structure of the ternary mixture. Nevertheless, the analysis of the amorphous structure in chapter 5 has shown that the ternary system exhibits a pronounced chemical and topological SRO which persists even at very high temperatures. The presence of this SRO could be related to the high glass forming ability of this system. An additional support to this observation is provided by experimental 114 and MD simulation results [78] for one of the strongest glass formers $\mathrm{SiO}_{2}$. In the MD simulation it is shown that the SRO (corresponding here to the open network structure) is omnipresent even at temperatures well obove the melting point, a feature which is not observed in fragile glasses.

It has been established by Inoue et al. [10] that crystallization of the glass forming alloy $\mathrm{Ni}_{25} \mathrm{Zr}_{55} \mathrm{Al}_{20}$, which exhibits a large $\Delta T_{x}(\approx 70 \mathrm{~K})$, takes place through a simultaneous precipitation of more than two types of precipitates. For a homogenous structure, such a crystallization process requires the redistribution of the constituents on a large range scale which is thought to represent a significant barrier for the transition to a crystalline phase.

This interpretation is in agreement with the stochastic approach proposed by Desré [115], who showed that an increase in the number of components in a glass forming liquid may contribute to inhibit intermetallic crystal nucleation, thus enhancing the glass forming ability. In the case of homogeneous nucleation, Desré found that addition of one further component lowers the probability of concentration fluctuations by a factor of the order of ten. 
Further, it has been reported by Matsubara et al. [75] that in the ternary systems $\mathrm{Ni}_{25} \mathrm{Zr}_{60} \mathrm{Al}_{15}$ and $\mathrm{Ni}_{20} \mathrm{La}_{55} \mathrm{Al}_{20}$ the atomic arrangements of $\mathrm{Al}$ atoms around $\mathrm{Zr}$ and $\mathrm{Ni}$ around La, respectively, differ markedly in the amorphous alloy from those in the crystallized state. This indicates that the environmental structure around Zr and La atoms, respectively, must be significantly changed for crystallization to occur, which provides the high thermal stability of the two ternary alloys.

The topological point of view [10, 116] is another concept to explain the stability of the amorphous phase by relating to the kinetic argument discussed above. It assumes that the high stability of the supercooled liquid and the improved properties of the amorphous solid originate to a certain amount from the increase in the packing density of the amorphous structure, which can be achieved by taking constituing elements with different atomic sizes. For instance, the intermediate atomic size of $\mathrm{Al}$ in the ternary alloys Ni-Zr-Al $\left(r_{Z r}>r_{A l}>r_{N i}\right)$ is presumed to be appropriate to fill vacant sites in the disordered structure consisting of $\mathrm{Zr}$ and $\mathrm{Ni}$ [4]. In the model used in this work, the experimental values of the atomic radii of the three constituing elements are well reproduced. Neverthless, the system exhibits an enhanced diffusivity by substituting $\mathrm{Ni}$ by $\mathrm{Al}$ atoms for a given temperature. The analysis of the atomic-level stress tensor in section 6.8 has shown that this effect is due to the n-defect (vacancy-like) character of the Al sites, which results in an increasing free volume by alloying $\mathrm{Al}$ to the binary $\mathrm{Ni}-\mathrm{Zr}$ alloy. This is clearly in contradiction with the topological argument above, according to which the free volume in $\mathrm{Ni}_{25} \mathrm{Zr}_{60} \mathrm{Al}_{15}$ should be smaller than in $\mathrm{Ni}_{35} \mathrm{Zr}_{65}$ and thus the mobility slower. This is a hint that in the determination of the packing density size differences play a secondary role compared to the difference in the binding strenght between the constituing elements. 


\section{Chapter 8}

\section{Summary}

Molecular dynamics (MD) simulations are used in this work to modell the bulk amorphous alloy $\mathrm{Ni}_{25} \mathrm{Zr}_{60} \mathrm{Al}_{15}$. The supercooled region of this alloy is known experimentally [4] to show a high thermal stability against cristallization and a low critical cooling rate compared with conventional metallic glasses. A reliable model for the interatomic couplings is first constructed. For this purpose we adopt the global scheme used by Hausleitner and Hafner [16] in their hybridized nearly-free-electron (NFE) tight-binding-bond (TBB) approach to interatomic forces in disordered (liquid and glassy) transition-metal alloys. We assume thus that the total energy of the system may be written as effective pairwise interactions augmented by a volume-dependent free-electron term $E_{V o l}$. We use for $E_{V o l}$ the energy expression of a homogeneous electron gas perturbed to first order by the ions as known from the theory of cohesion of simple metals [60]. Concerning the effective pair potentials, a hybrid of different approaches is adopted. For the $\mathrm{Ni}-\mathrm{Zr}$ subset we use the pair potentials as derived by Hausleitner and Hafner [56] for the amorphous binary alloy $\mathrm{Ni}_{35} \mathrm{Zr}_{65}$ in the framework of the NFE-TBB scheme. The pair interaction $\Phi_{A l A l}$ is described in our model by the pair potential derived for pure $\mathrm{Al}$ within the framework of the second order pseudopotential theory [17]. The remaining cross-term interactions $\Phi_{N i A l}$ and $\Phi_{Z r A l}$ are assumed to have the empirical form of Stillinger-Weber [18] with parameters which are fitted to experimental data of the crystalline structures $\mathrm{Ni}_{3} \mathrm{Al}$ and $\mathrm{Zr}_{3} \mathrm{Al}$, respectively.

Comparison of the calculated radial distribution functions (RDF) with the experimental results of Matsubara et al. [75] shows that the model reproduces the structural features of amorphous $\mathrm{Ni}_{25} \mathrm{Zr}_{60} \mathrm{Al}_{15}$ with a good accuracy. Moreover, our model is able to predict the experimental GT temperature $T_{g}$ with an accuracy of about $15 \%$. The activation energies $Q$ for diffusion in the glassy state are obtained in the range of those given by experimental measurements.

In chapter 4, analysis of the thermodynamics shows that the caloric GT temperature $T_{g}$ is shifted to lower temperatures by alloying $\mathrm{Al}$ to Ni-Zr alloy. This effect is attributed to the larger mixing tendency of the Zr-Ni subsystem compared 
with the subsystem $\mathrm{Zr}-\mathrm{Al}$, as can be seen from the representation of the different interatomic couplings in section 4.4. Upon undercooling, the binary $\mathrm{NiZr}_{2}$ and the ternary $\mathrm{Ni}_{25} \mathrm{Zr}_{60} \mathrm{Al}_{15}$ melts fall out of equilibrium at $T \approx 1200 \mathrm{~K}$ and $T \approx 1025 \mathrm{~K}$, respectively. These temperatures lie well above the experimental glass temperature $T_{g, \exp }$ from DSC measurements (about $652 \mathrm{~K}$ [104] and $720 \mathrm{~K}$ [4], respectively). A great part of this discrepancy seems to be due to the extrem large cooling rate used in our simulations $\left(\approx 10^{10} \mathrm{~K} / \mathrm{s}\right)$ compared to typical cooling rates used in experiments $\left(<10^{6} \mathrm{~K} / \mathrm{s}\right)$.

The structural analysis in chapter 5 reveals the existence of well-defined prepeaks in the Faber-Ziman Structure factors $S_{N i N i}^{F Z}(q)$ and $S_{A l A l}^{F Z}(q)$. Both reflect the existence of pronounced chemical and topological order. The Ni-prepeak is associated with the weak Ni-Ni coordination in favour of the strong Ni-Zr heterocoordination, because of the good mixing behavior in the binary system Ni-Zr. The corresponding length of $\approx 3.3 \AA$ is related to the second nearest $\mathrm{Ni}-\mathrm{Ni}$ pair correlation. The prepeak in $S_{A l A l}^{F Z}(q)$ is associated with a tendency of Al-Al clustering, as can be deduced from the anomalous pronounced first peak in $g_{A l A l}$. The corresponding concentration fluctuations have a length scale of about $5.8 \AA$. The clustering tendency of $\mathrm{Al}$ atoms seems to be a result of the stronger mixing behavior of $\mathrm{Ni}-\mathrm{Zr}$ system relatively to the $\mathrm{Zr}-\mathrm{Al}$ one, i.e. the $\mathrm{Al}-\mathrm{Zr}$ heterocoordination preference is depressed by a stronger $\mathrm{Ni}$-Zr preference. Moreover, the smaller Ni atoms have a more appropriate size to match in the Bernal holes of the Zr matrix than the Al atoms.

A study of the bond-angle distribution in section 5.4 shows that the angular correlations at $\mathrm{Ni}$ and $\mathrm{Zr}$ sites are compatible with a local trigonal prismatic order, while the $\mathrm{Al}$ atoms occupy sites with a predominatly icosahedral symmetry. This suggests that the structure of the ternary alloy consists of a network of Ni-Zr units having a trigonal prismatic topology. The Ni atoms occupy the centers of the prisms and the $\mathrm{Al}$ atoms are constrained to group in the space between these Ni-Zr units.

From the temperature dependence of the prepeaks we deduce that they exist even at temperatures as high as $T=5000 \mathrm{~K}$. This indicates that the structural organization develops at these very high temperatures already. This contrasts with the conventional picture that the atoms in a melt are randomly distributed. The persistence of a well-defined SRO even far obove the melting temperature seems to be a common feature of glass formers with bonding forces of partial covalent nature, like network-forming and transition-metals glassy systems.

In chapter 6 several aspects of the dynamics are studied. The analysis of the van Hove correlation functions in section 6.2 shows that the onset of hopping processes actually takes place in a temperature range well above the kinetic GT temperature $T_{c}(T>1400 \mathrm{~K})$. In this temperature range, the transport mechanism seems to change from a flow-like motion to a hopping-like one. The pronounced peak at the origin in the van Hove distinct correlation function gives evidence of the highly correlated nature of the hopping processes. This features seems, however, primarily 
to concern the $\mathrm{Ni}$ and $\mathrm{Al}$ atoms. For $\mathrm{Zr}$ atoms, the flow-like motion seems to be the dominant transport mode even in the region of the kinetic GT and below, at least for the configurations accessible by the present cooling treatment.

The onset of the hopping processes seems to be related to decoupling effects observed in the same temperature range: decoupling of the diffusivity and the density relaxation, on one hand, and diffusivity and viscosity (or the breakdown of the Einstein-Stokes relation), on the other hand. Moreover, a kink in the temperature dependence of the volume of the cell has been observed when these decouplings take place. Although there is evidence that these effects are related, the nature of this relation is not well understood. In order to clear this relation, further investigated still are necessary. The role of dynamic heterogeneities has to be considered particularly.

We observe an enhanced diffusivity (or equivalently a decreased viscosity) by substituting $\mathrm{Ni}$ by $\mathrm{Al}$ atoms for a given temperature. The analysis of the atomic-level stress tensor in section 6.8 shows that this effect is due to the n-defect (vacancy-like) character of the $\mathrm{Al}$ sites, which results in an increasing free volume by alloying $\mathrm{Al}$ to the binary $\mathrm{Ni}-\mathrm{Zr}$ alloy. This is clearly in contradiction with the topological argument advanced by Inoue and co-workers [10], according to which the high thermal stability of Zr-rich Ni-Zr-Al ternary alloys relatively to Zr-rich binary ones originates from the increase of the packing fraction by the dissolution of Al. From our model, it comes out that in the determination of the packing density, size differences play a secondary role compared to the difference in the binding strength between the constituent. 


\section{Appendix A}

\section{k-dependent shear viscosity}

\section{A.1 Definition}

Owing to its intrinsic hydrodynamic nature, the shear viscosity coefficient $\eta$ is the quantity used to specify transverse collective motions in a fluid on a macroscopic scale. The associated dynamical quantity is the transverse current correlation function [89, 95]

$$
C_{t}(k, t)=\frac{1}{N}\left\langle j_{\mathbf{k}}^{x}\left(t+t_{0}\right) j_{-\mathbf{k}}^{x}\left(t_{0}\right\rangle_{t_{0}},\right.
$$

$\mathbf{j}_{\mathbf{k}}(t)$ is the space Fourier transform of the particle current

$$
\mathbf{j}(\mathbf{r}, t)=\sum_{i=1}^{N} \mathbf{v}_{i}(t) \delta\left[\mathbf{r}-\mathbf{r}_{i}(t)\right],
$$

where $\mathbf{r}_{i}$ and $\mathbf{v}_{i}$ are the position and velocity of particle $i$ respectively. In eq.A.1, the $z$-axis is chosen as the direction of the wave vector $\mathbf{k}$.

The shear viscosity $\eta$ is related to the long-wavelenght, low frequency behavior (hydrodynamic limit) of the spectrum of $C_{t}(k, t)$ by

$$
C_{t}(k, t)=\frac{\pi \rho m^{2}}{k_{B} T} \lim _{\omega \rightarrow 0} \lim _{k \rightarrow 0} \frac{\omega^{2}}{k^{2}} C_{t}(k, \omega),
$$

where $\rho=N / V$ is the number density and $\mathrm{m}$ is the atomic mass.

Beyond the strict hydrodynamic regime, a liquid is found to support shear-wave propagation over short distances, manifesting itself in the appearance of inelastic peaks in the transverse current spectrum. These non-hydrodynamic features are described formally by a wavevector- and frequency-dependent shear viscosity coefficient, which can be introduced in the framework of the memory-function formalism 89 .

In the following we consider the normalized transverse current $\rho_{t}(k, t)=$ $\left(C_{t}(k, t) / C_{t}(k, 0)\right.$. In terms of Laplace transforms $\tilde{f}(z)=L f(t)$ one finds that

$$
\tilde{\rho}_{t}(k, z)=\left[z+\tilde{K}_{t}(k, z)\right]^{-1}=\left[z+\left(k^{2} / \rho m\right) \tilde{\eta}(k, z)\right]^{-1}
$$


$K_{t}(k, t)=L^{-1} \tilde{K}_{t}(k, z)$ is called the transverse current memory function. The quantity $\tilde{\eta}(k, z=i \omega)$ is interpreted as the wavevector- and frequency-dependent shear viscosity. At finite frequencies, $\tilde{\eta}(k, z=i \omega)$ is in general a complex quantity. Its imaginary part describes shear-wave propagation while the real part accounts for damping effects. As $\omega \rightarrow 0, \tilde{\eta}(k, i \omega)$ becomes purely real and it is reasonable to describe the microscopic low-frequency response of the system by a generalized shear viscosity coefficient

$$
\eta(k) \equiv \lim _{\omega \rightarrow 0} \tilde{\eta}(k, i \omega),
$$

or equivalently

$$
\eta(k)=\rho m / k^{2} \int_{0}^{+\infty} K_{t}(k, t) d t .
$$

In particular, $\eta(k)$ approaches the shear viscosity $\eta$ at large $k$

$$
\lim _{k \rightarrow 0} \eta(k)=\eta .
$$

Writing

$$
\begin{gathered}
\tilde{\rho}_{t}(k, z=i \omega)=\rho_{t}^{\prime}(k, \omega)+i \rho_{t}^{\prime \prime}(k, \omega), \\
\rho_{t}^{\prime}(k, \omega)=\int_{0}^{+\infty} \cos (\omega t) \rho_{t}(k, t) d t=\pi \rho_{t}(k, \omega), \\
\rho_{t}^{\prime \prime}(k, \omega)=-\int_{0}^{+\infty} \sin (\omega t) \rho_{t}(k, t) d t
\end{gathered}
$$

along with similar definitions for $\tilde{K}_{t}(k, z=i \omega)$, it is easy to deduce that

$$
K_{t}^{\prime}(k, \omega)=\frac{\rho_{t}^{\prime}(k, \omega)}{\left[\rho_{t}^{\prime}(k, \omega)\right]^{2}+\left[\rho_{t}^{\prime \prime}(k, \omega)\right]^{2}} .
$$

To evaluate $\eta(k)$, one first determines numerically $\rho_{t}^{\prime}(k, \omega)$ and $\rho_{t}^{\prime \prime}(k, \omega)$ from the data of the transverse current correlation function $\rho_{t}(k, t)$, and then $K_{t}^{\prime}(k, \omega)$ by eq.A.11; an inverse Fourier transform gives $K_{t}(k, t)$. The integral of eq. A.11) gives finally $\eta(k)$.

\section{A.2 Results}

The MD analysis of the k-dependent $\eta(k)$ has been performed for the binary system $\mathrm{Ni}_{35} \mathrm{Zr}_{65}$ by use of the Hausleitner-Hafner interatomic couplings (see chapter 3 ) and with a total particles number of $N=648 . C_{t}(k, t)$ has been calculated at different temperatures for a range of $k$ larger than the minimum value $k_{\text {min }}=2 \pi / L$, which 
is given by the box lenght $L$ of the system. Since $C_{t}(k, t)$ is a collective property, no distinction is made between $\mathrm{Ni}$ and $\mathrm{Zr}$ in eq. A.1). The statistical accuracy of eq. (A.1) is affected by the lack of averaging over the particles. This has been compensated by averaging $C_{t}(k, t)$ over a large number of configurations (about $2 \times 10^{5}$ ) in addition to the six combinations offered by eq. A.1 to chose the longitudinal and the transversal directions. The k-dependent viscosity $\eta(k)$ has been then numerically evaluated according to the method described in the previous section. The results are displayed in fig. A.1A.2.

At higher temperatures above approximatly $1400 K, \eta(k)$ for $k \rightarrow 0$ approximates well the values directly computed by using the Green-Kubo relations (see section 6.6), additionally indicated in the figures by the triangles. This provides an explicit support to the reliability of the Green-Kubo relation in calculating the shear viscosity even for systems with rather limited size. At lower temperatures $\eta(k)$ exhibits a strong behavior in the vicinity of $k_{\min }=2 \pi / L$, so that an extrapolation to $k \rightarrow 0$ becomes difficult. 


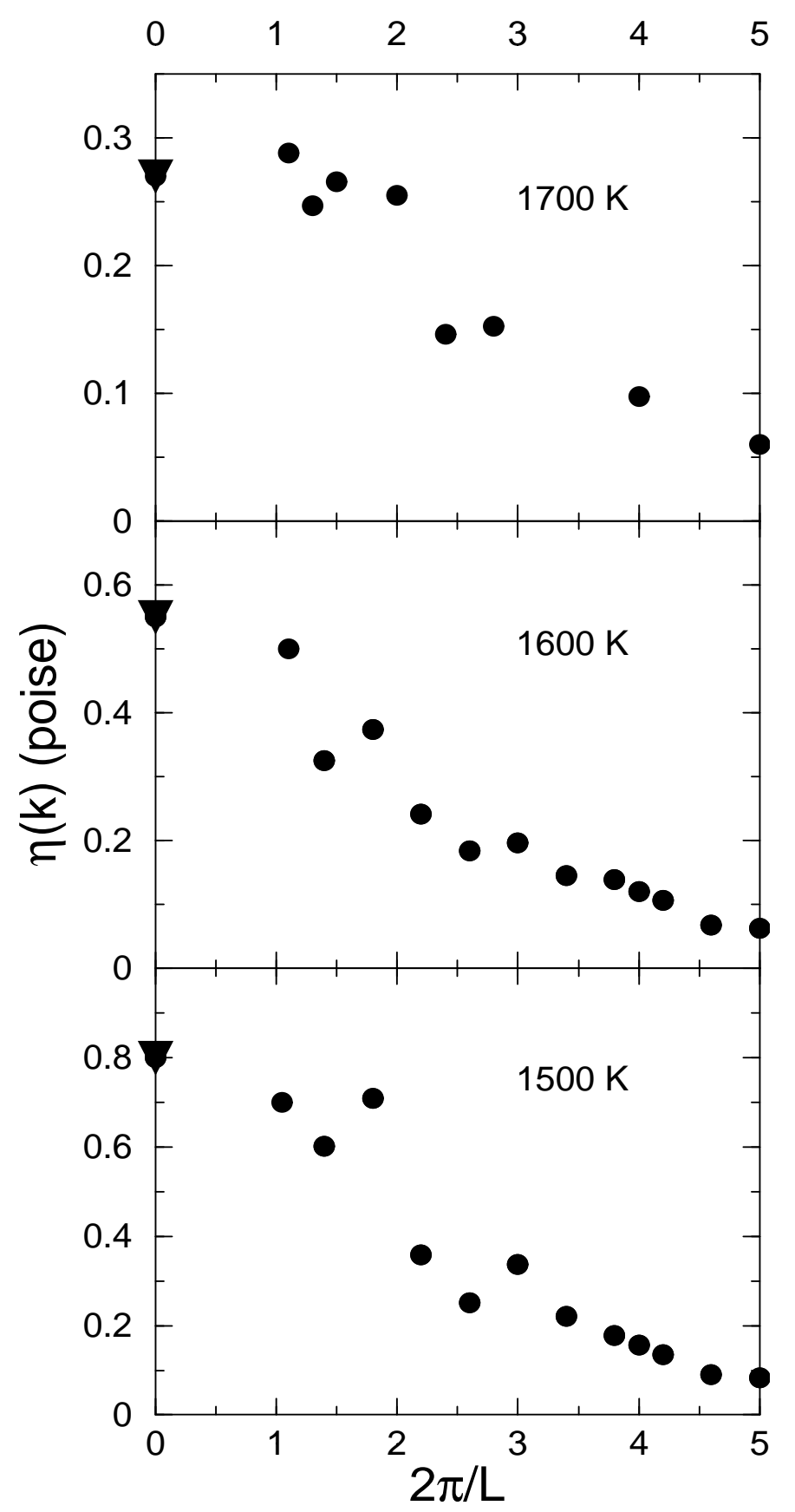

Figure A.1: k-dependent shear viscosity $\eta(k)$ in binary $\mathrm{Ni}_{35} \mathrm{Zr}_{65}$. The triangles at $k=0$ are the values of the shear viscosity $\eta$ calculated from the Green-Kubo relation. $\mathrm{k}$ values are in units of $2 \pi / L$. 


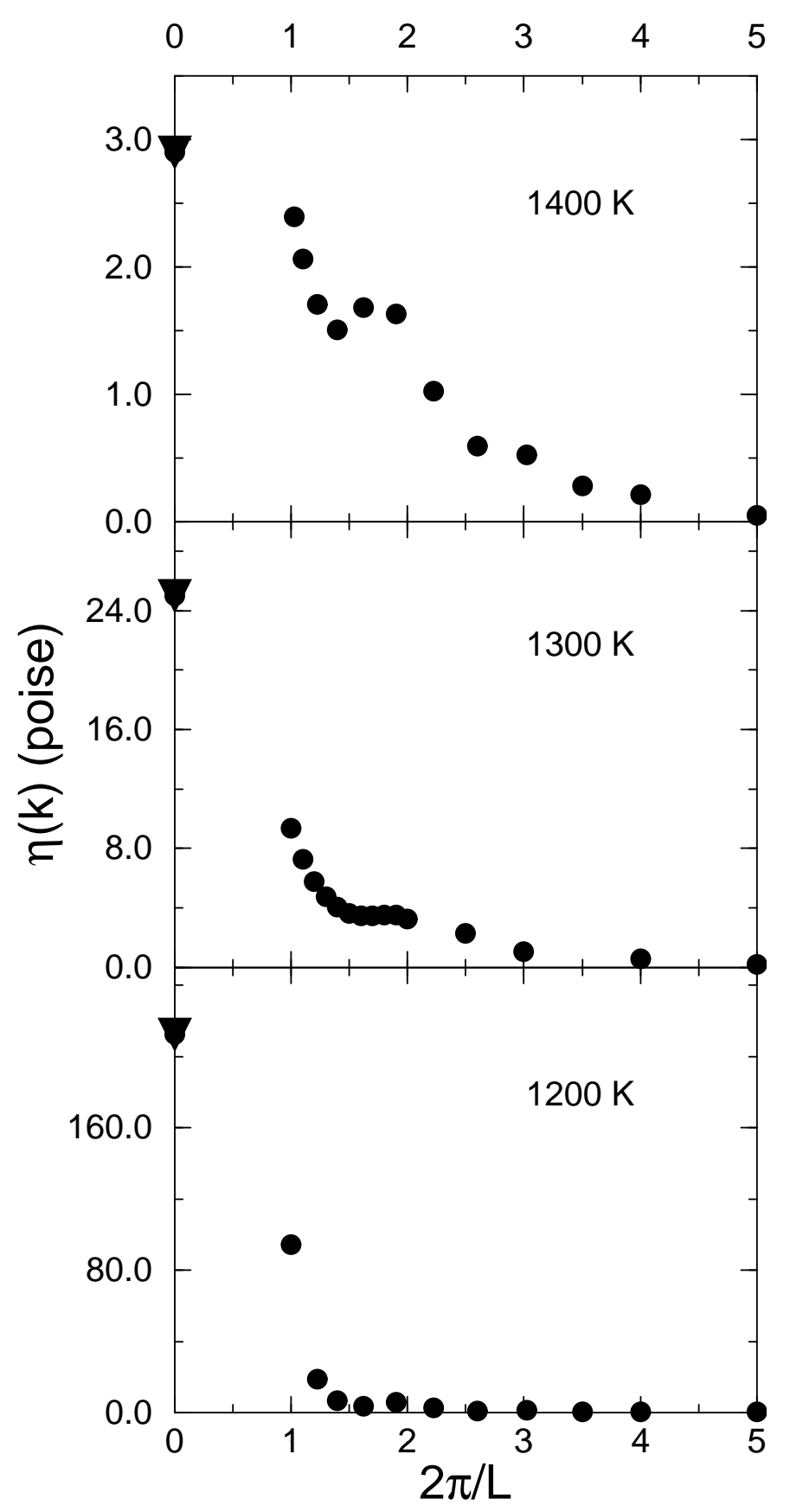

Figure A.2: As in fig. A.1, but for lower temperatures. 


\section{Appendix B}

\section{Fitting results}

Table B.1: Experimental values of $\mathrm{Ni}_{3} \mathrm{Al}$ and $\mathrm{Zr}_{3} \mathrm{Al}$ properties used in the fit of the parameters of the Stillinger-Weber pair-potentials $\Phi_{N i A l}$ and $\Phi_{Z r A l}$ respectively.

\begin{tabular}{|c|c|c|c|c|}
\hline & \multicolumn{2}{|c|}{$\mathrm{Ni}_{3} \mathrm{Al}$} & \multicolumn{2}{|c|}{$\mathrm{Zr}_{3} \mathrm{Al}$} \\
\hline & exp. & calc. & exp. & calc. \\
\hline$a[\AA]$ & 3.57 [117] & 3.54 & 4.37 [118 & 4.38 \\
\hline$E_{c}[\mathrm{eV}]$ & 4.57 [117 & 4.45 & 5.8963 & 5.90 \\
\hline$B\left[\mathrm{eV} . \AA^{-3}\right]$ & 1.03 [117 & 1.05 & 0.58 & 0.65 \\
\hline
\end{tabular}

To get the experimental cohesive energy of the alloys one adds the formation enthalpy to the cohesive energy of an ideal mixture, obtained by using the pure metal cohesive energies.

Since an experimental value of the bulk modulus of $\mathrm{Zr}_{3} \mathrm{Al}$ is not available in the literatur (to our knowledge), we used the concentration-weighted average of the bulk modulus of pure $\mathrm{Zr}$ [119] and $\mathrm{Al}$ [117].

The bulk modulus were calculated from the second derivative of the atomic cohesive energy $E_{c}$ with respect to the atomic volume $\Omega$ [120]

$$
B=\Omega \frac{d^{2} E_{c}}{d V^{2}}
$$




\section{Bibliography}

[1] R. W. Cahn, in Glasses and Amorphous Materials, J. Zarzycki, Ed., vol. 9 of Materials Sciences and Technology, edited by R. W. Cahn, P. Haasen, and E. J. Kramer (VCH Press, Weinheim, 1991), pp. 493-548.

[2] A. L. Greer, Science 267, 1947 (1995).

[3] T. Masumoto, Sci. Rep. RITU A 39, 91 (1994); A. Inoue, Mater. Trans., JIM, 36, 866 (1995).

[4] A. Inoue, T. Zhang, T. Masumoto, Mater. Trans., JIM 31, 177 (1990).

[5] A. Inoue, T. Zhang, T. Masumoto, Mater. Trans., JIM 30, 965 (1989).

[6] T. Zhang, A. Inoue, T. Masumoto, Mater. Trans., JIM 32, 1005 (1991).

[7] A. Peker und W. L. Johnson, Appl. Phys. Lett. 63, 2342 (1993).

[8] E. Bakke, R. Busch, W. L. Johnson, Appl. Phys. Lett. 67, 3260 (1995).

[9] R. Busch, A. Masuhr, E. Bakke, W. L. Johnson, Mater. Sci. Forum 269-272, 547 (1998).

[10] A. Inoue, T. Zhang, T. Masumoto, J. Non-cryst. Solids 156-158, 473 (1993).

[11] H. Teichler, phys. stat. solidi. (b) 172, 325 (1992).

[12] H. Teichler, Phys. Rev. E 53, 4287 (1996).

[13] H. Teichler, Phys. Rev. Letters 76, 62 (1996).

[14] B. Böddeker, H. Teichler, Phys. Rev. E 59, 1948 (1999).

[15] H. Teichler, Phys. Rev. B 59, 8473 (1999).

[16] Ch. Hausleitner und J. Hafner, Phys. Rev. B 45, 115 (1992).

[17] D. G. Pettifor, M. A. Ward, Solid State Commun. 49, 291 (1984).

[18] F. H. Stillinger, T. A. Weber, Phys. Rev. B 31, 5262 (1985).

[19] R. Busch, E. Bakke, W. L. Johnson, Acta mater. 46, 4725 (1998). 
[20] R. Busch, R. Schneider, S. Peker, W. L. Johnson, Appl. Phys. Lett. 67, 1544 (1995).

[21] W. Frank, Def. Diff. Forum 143-147, 695 (1997); A. Grandjean, Y. Limoge, Acta mater. 45, 1585 (1997).

[22] X.-P. Tang, U. Geyer, R. Busch, W. L. Johnson, Y. Wu, Nature 402, 160 (1999).

[23] G. Frohberg, Def. Diff. Forum 143-147, 869 (1997).

[24] W. Götze, L. Sjoegren, Transp. Theory Stat. Phys. 24, 801 (1995).

[25] L. J. Lewis, G. Wahnström, Phys. Rev. E 50, 3865 (1994).

[26] W. Kob, H. C. Andersen, Phys. Rev. E 51, 4626 (1995).

[27] W. Kob, H. C. Andersen, Phys. Rev. E 524134 (1995).

[28] J. N. Roux, J. L. Barrat, J. P. Hansen, J. Phys. C 1, 7171 (1989).

[29] H. Miyagawa, Y. Hiwatari, Phys. Rev. A88, 8287 (1991).

[30] H. Teichler, Def. Diff. Forum 143-147, 717 (1997).

[31] H. J. Höfler, R. S. Averback, G. Rummel, H. Mehrer, Phil. Mag. Lett. 66301 (1992).

[32] F. Faupel, P. W. Hüppe, K. Rätzke, Phys. Rev. Lett. 65, 1219 (1990).

[33] Vorlesungsmanuskripte des 20. IFF-Ferienkurses in der KFA Jülich, 13 - 24 February 1989, Computersimulation in der Physik.

[34] Proc. of Intern. School of Phys. "Enrico fermi": Molecular Dynamics Simulation in Statistical Mechanical Systems, edited by G. Ciccoti and W. G. Hoover (North Holland 1986).

[35] J. Stadler, R. Mikulla, H.-R. Trebin, Int. J. Mod. Phys. C 8, 1131 (1997).

[36] B. Böddeker, Dissertation, Georg-August Universität zu Göttingen, 1999.

[37] B. Böddeker and H. Teichler in Molecular Dynamics on Parallel Computers (World Scientific Publishing Co. Pte. Ltd., Forschungszentrum Jülich 8 - 10 february 1999, 1999)

[38] M. P. Allen, D. J. Tildesly, Computer Simulation of Liquids (Clarendon Press, Oxford 1987).

[39] D. W. Heermann, Computer Simulation Methods in Theoretical Physics (Springer 1987). 
[40] R. Haberlandt, S. Fritzsche, G. Peinel, and K. Heinzinger, Molekulardynamik (Vieweg, Braunschweig/Wiesbaden, 1995).

[41] H. Teichler, unpublished. The method is described in Ref. [42].

[42] B. Kiefer, Diplomarbeit, Georg-August Universität zu Göttingen, 1993.

[43] C. W. Gear, Numerical Initial Value Problems in Ordinary Differential Equations (Prentice-Hall, Englewood Cliffs, N.J. 1971).

[44] L. V. Woodcock, Chem. Phys. Lett. 10, 257 (1971).

[45] S. Nosé, Progr. Theoretical Physics Suppl. 103, 1 (1991).

[46] H. C. Andersen, J. Chem. Phys. 72, 2384 (1980).

[47] M. Parrinello, A. Rahman, Phys. Rev. Lett. 45, 1196 (1980).

[48] M. Manninen, R. M. Nieminen, M. J. Puska, in many-Atom Interactions in Solids, edited by R. M. Nieminen, M. J. Puska, and M. Manninen (Springer, Berlin, Heidelberg 1990), p. 2.

[49] W. A. Harrison, Pseudopotentials in the Theory of Metals (Benjamin, New York 1966).

[50] H. Hohenberg, W. Kohn, Phys. Rev. B 136, 864 (1964).

[51] J. K. Nørskov, N. D. Lang, Phys. Rev. B 21, 2131 (1980).

[52] M. S. Daw, M. I. Baskes, Phys. Rev. Lett. 50, 1285 (1983).

[53] M. W. Finnis, J. R. Sinclair, Phil. Mag. A 50, 45 (1986).

[54] J. A. Moriarty, Phys. Rev. B 38, 3199 (1988), J. A. Moriarty, Mike Widom, Phys. Rev. B 56, 7905 (1997).

[55] U. Rössler, Dissertation, Georg-August Universität zu Göttingen, 1995.

[56] Ch. Hausleitner, J. Hafner, J. Phys.: Condens. Matter 2, 6651 (1990); Ch. Hausleitner, J. Hafner, Phys. Rev. B 45, 115 (1992).

[57] Ch. Hausleitner, J. Hafner, Phys. Rev. B 45, 128 (1992).

[58] H. Wills, A. Harrison, Phys. Rev. B 28, 4364 (1983).

[59] U. Rössler, H. Teichler, Phys. Rev. B 61, 394 (2000).

[60] M. W. Finnis, J. Phys. F 4, 1645 (1974). 
[61] J. Hafner, From Hamiltonians to Phase Diagrams, Springer Series in SolidState Sciences, Bd. 70 (Springer, Berlin, Heidelberg 1987).

[62] NAG Fortran Library Manual Mark 8, 1980 (NAG Central Office, 7 Banbury Road, Oxford OX26NN, U.K.), Chapter F01.

[63] Cohesion in metals, edited by F. R. de Bor, R. Boom, W. C. M. Mattens, A. R. Miedema and A. K. Niessen (North Holland, Amsterdam 1988).

[64] T. Fukunaga, N. Hayashi, N. Watanabe, K. Suzu Rapidly Quenched Metals, edited by S. Steeb and H. Warlimont (Elsevier, N. Y. 1985), p. 475.

[65] M. Maret, T. Pomme, A. Pasturel, P. Chieux, Phys. Rev. B 42, 1598 (1990).

[66] L. D. Landau, E. M. Lifshitz, Statistical Physics, 3rd ed. (Pergamon, N. Y. 1980).

[67] J. R. Ray, Comput. Phys. Rep. 8, 109 (1988).

[68] C. A. Angell, in Structure and Mobility in Molecular and Atomic Glasses, edited by J. M. O’Reilly and M. Goldstein, Ann. N.Y. Acad. Sci. 371, 136 (1981).

[69] K. Ohsaka, S. K. Chung, W. K. Rhim, Acta mater. 46, 4535 (1998).

[70] Y. Waseda, The Structure of Non-Crystalline Materials, (McGraw-Hill, N. Y. 1980)

[71] S. Lele, in Metallic Glasses, edited by T. R. Anantharaman, Trans Tech Publications (1984)

[72] J. D. Bernal, Nature 183, 141 (1959).

[73] D. E. Polk, J. Non-Cryst. Solids 5, 365 (1971).

[74] P. H. Gaskell, J. Non-Cryst. Solids 32, 207 (1979).

[75] E. Matsubara, Y. Waseda, Mater. Trans., JIM 36, 883 (1995).

[76] T. Mizoguchi, S. Yoda, N. Akutsu, S. Yamada, J. Nishioka, T. Suemasa, N. Watanabe, in Rapidly Quenched Metals (Ref. 64), p. 483.

[77] A. B. Mutiara Q. N., Dissertation, Georg-August Universität zu Göttingen, 1999; A. B. Mutiara Q. N., Korrelationsfunktionen und Memory-Funktionen in MD-simulierten glasbildenden Ni-Zr-Schmelzen (Cuvellier Verlag, Göttingen 2000).

[78] J. Horbach, W. Kob, Phys. Rev. B 60, 3169 (1999). 
[79] P. A. Duine, S. K. Wonnell, J. Siestma, Mat. Sci. Engn. A 179/180, 270 (1994).

[80] G. Rummel, H. Mehrer, phys. stat. Sol. (b) 185, 327 (1994).

[81] A. Heesemann, K. Rätzke, F. Faupel, J. Hoffmann, K. Heinemann, Europhys. Lett. 29, 221 (1995); E. Ehmler, A. Heesemann, K. Rätzke, F. Faupel, U. Geyer, Phys. Rev. Lett. 80, 4919 (1998).

[82] J. O. Hirschfelder, C. F. Curtiss, R. B. Bird, Molecular Theory of Gases and Liquids (Wiley \& Sons, New York 1954).

[83] W. Kob, J. Phys.: Condens. Matter 11, R85 (1999).

[84] J.P. Hansen, S. Yip, Transp. Theory Stat. Phys. 24, 1149 (1995).

[85] W. Kauzmann, Chem. Rev.43, 219 (1948).

[86] C. A. Angell, J. Non-Cryst. Solids 131-133, 13 (1991).

[87] R. S. Averback, Mater. Res. Bull. 16, 47 (1991).

[88] H. Hahn, S. Flege (unpublished).

[89] J. P. Hansen, I. R. McDonald, Theory of Simple Liquids (Academic Press, London 1986); J. P. Boon, S. Yip, Molecular Hydrodynamics (Mc Graw Hill, New York 1980); U. Balucani, M. Zoppi, Dynamics of the Liquid State (Oxford Science Publications, Oxford 1994).

[90] H. Sieber, Diplomarbeit, Georg-August Universität zu Göttingen, 1992.

[91] C. Gaukel, H. R. Schober, Solid. State Commun. 107, 1 (1998).

[92] C. Gaukel, Dissertation, Rheinisch-Westfälische Technische Hochschule Aachen, 1998.

[93] K. L. Ngai, in Non-Debye Relaxations in Condensed Matter, edited by T. V. Ramakrishnan and M. Raj Laksmi (World Scientific, Singapore 1986), p. 171.

[94] D. N. Perera, P. Harrowell, Phys. Rev. Lett. 81, 120 (1998).

[95] U. Balucani, R. Vallauri, T. Gaskell, Phys. Rev. A 35, 4263 (1987).

[96] B. J. Palmer, Phys. Rev. E 49, 359 (1994).

[97] E. Rössler. Phys. Rev. Lett. 65, 1595 (1990); F. Fujara, B. Geil, H. Sillescu, G. Fleischer, Z. Phys. B 88, 195 (1992).

[98] U. Geyer, S. Schneider, Y. Qiu, M. P. Macht, T. A. Tombrello, W. L. Johnson, Mat. Science Forum 235-238, 343 (1997). 
[99] D. Thirumalai, R. D. Mountain, Phys. Rev. E 47, 479 (1993).

[99] F. Wenwer, K. Knorr, M.-P Macht, H. Mehrer, Def. Diff. Forum 143-147, 831 (1997).

[100] K. Knorr, Dissertation, Universität Münster, 1999.

[101] U. Geyer, S. Schneider, W. L. Johnson, Y. Qiu, T. A. Tombrello, M. P. Macht, Phys. Rev. Lett. 75, 2364 (1995).

[102] S. K. Sharma, F. Faupel, J. Mater. Res. 14, 3200 (1999).

[103] T. Egami, K. Maeda, V. Vitek, Philos. Mag. A 41, 883 (1980); D. Srolovitz, K. Maeda, V. Vitek, T. Egami, Philos. Mag. A 44, 847 (1981).

[104] Z. Altounian, T. Guo-hua, J. O. Strom-Olsen, J. Appl. Phys. 54 (1983).

[105] R. Lück, Qing Jiang, B. Predel, J. Non-Cryst. Solids 117-118, 911 (1990).

[106] M. T. Cicerone, M. D. Ediger, J. Chem. Phys. 104, 7210 (1996).

[107] J. L. Barrat, J. N. Roux, J. P. Hansen, Chem. Phys. 149, 197 (1990); C.Z.-W. Liu, I. Oppenheim, Phys. Rev. E 53, 799 (1996); R. Yamamoto, A. Onuki, Phys. Rev. Lett. 81, 4915 (1998).

[108] F. H. Stillinger, J. A. Hodgdon, Phys. Rev. E 50, 2064 (1994).

[109] R. Richert, A. Blumen, in Disordered Effects on Relaxational Processes, edited by R. Richert and A. Blumen (Springer-Verlag, Berlin 1994), p. 1.

[110] W. Weiss, M. Moske, K. Samwer, Phys. Rev. B 58, 9062 (1998).

[111] C. Donati, J. F. Douglas, W. Kob, S. J. Plimpton, Peter H. Poole, S. C. Glotzer, Phys. Rev. Lett. 80, 2338 (1998).

[112] R. Yamamoto, A. Onuki, Europhys. Lett. 40, 61 (1997); D. N. Perera, P. Harrowell, J. Chem. Phys. 111, 5441 (1999).

[113] G. Adam, J. H. Gibbs, J. Chem. Phys. 43, 139 (1965).

[114] D. L. Price, Carpenter, J. Non-Cryst. Solids 92, 153 (1987).

[115] P. J. Desré, Mater. Trans., JIM 38, 583 (1997).

[116] X. H. Lin, W. L. Johnson, J. Appl. Phys. 78, 6514 (1995).

[117] S. P. Chen, A. F. Voter, D. J. Srolovitz, Scripta Metall. 20, 1389 (1986).

[118] P. Villars, L. D. Calvert (Eds.), Pearson's Handbook of Crystallographic Data for Intermetallic Phases, Amer. Soc. for Metals (Metal Park Ohio, 1986). 
[119] G. Simmons, H. Wang, Single Crystal Elastic Constants and Calculated Aggregates Properties (MIT Press, Cambridge, Mass. 1971).

[120] B. Ihmels, Diplomarbeit, Georg-August Universität zu Göttingen, 1993.

[121] V.V. Sokolovskij, Theorie der Plastizität (VEB Verlag, Berlin 1955).

[122] R. J. Meyer, Dissertation, Gerhard-Mercator-Universität - Gesamthochschule Duisburg, 1998.

[123] see, e.g., R. Brüning, K. Samwer, Phys. Rev. B 46, 11318 (1992).

[124] K. Vollmayr, W. Kob, K. Binder, Phys. Rev. B 54, 15808 (1996).

[125] P.H. Gaskell, in Glassy Metals II, edited by H. Beck and H.-J Güntherodt (Springer-Verlag, Berlin 1983), p. 23.

[126] K. Suzuki, T. Fukunaga, K. Shibata, T. Otomo, H. Mizuseki, in Thermodynamics of Alloy Formation, edited by Y. A. Chang and F. Sommer (The Minerals, Metals and Materials Society, 1997). 


\section{Danksagung}

In erster Linie gilt mein Dank Herrn Prof. Teichler für die Anregung, Förderung und Betreuung dieser Arbeit, sowie seine unendlich grosse Diskussionsbereitschaft.

Herrn Prof. Dr. K. Samwer sei herzlich gedankt für seine Tätigkeit als Korreferent.

Ingeborg und den Mitgliedern der Arbeitsgruppe Theorie: Jörg, Kevin und Boris; sowie den ausgeschiedenen Mitgliedern: Bert, Benny, Matthias, Florian, und Martin danke ich für das angenehme Arbeitsklima im Labor. An die vielfältigen Diskussionen bei einer duftenden Tasse Kaffee aus der besten Bohne werde ich mich noch lange erinnern. Die Hilfsbereitschaft von Jörg bei jedem Computerproblem bleibt vorbildlich. Man kann bei ihm aber noch viel mehr lernen, besonders wenn ihm der Kaffee schmeckt. Kevin bin ich dankbar für sein Talent, gute Atmosphäre um sich herum zu verbreiten. Ich danke ihm auch für die vielen schönen Diskussionen, über Physik inbegriffen, u.a. am Marktplatz in den leichten Tagen des Frühlings 2000. Mit der Gesellschaft von Benny, sei es beim Unterhalten oder zusammen rauchen, verbinde ich schöne Erinnerungen. Meine erste MD simulation habe ich mit seiner Hilfe gestartet.

Ein Besonderer Dank gilt Maria Dolores, die mich bei dieser Arbeit begleitet und unterstüzt hat. 